\title{
Characterization of mitochondrial glycerol-3-phosphate acyltransferase in notothenioid fishes
}

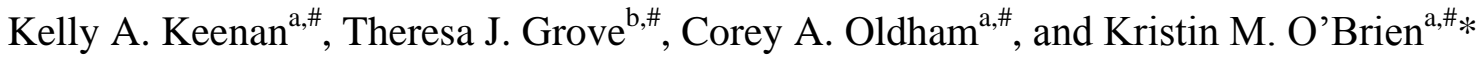 \\ ${ }^{a}$ University of Alaska Fairbanks, Institute of Arctic Biology, Fairbanks, AK, 99775 \\ ${ }^{\mathrm{b}}$ Department of Biology, Valdosta State University. Valdosta, GA 31698 \\ \# these authors contributed equally to this work \\ *corresponding author \\ University of Alaska Fairbanks \\ Institute of Arctic Biology, P.O. Box 757000, Fairbanks, AK 99775 \\ Ph: 907-474-5311 \\ Fax: 907-474-7666 \\ Email: kmobrien@alaska.edu
}

Keywords: mitochondria, phospholipids, fishes, cold adaption 


\subsection{ABSTRACT}

Hearts of Antarctic icefishes (suborder Notothenioidei, family Channichthyidae) have higher densities of mitochondria, and mitochondria have higher densities of phospholipids, compared to red-blooded notothenioids. Glycerol-3-phosphate acyltransferase (GPAT) catalyzes the ratelimiting step in glycerolipid biosynthesis. There are four isoforms of GPAT in vertebrates; GPAT1 and GPAT2 are localized to the outer mitochondrial membrane, whereas GPAT3 and GPAT4 are localized to the endoplasmic reticulum membrane. We hypothesized that transcript levels of GPAT1 and/or GPAT2 would mirror densities of mitochondrial phospholipids and be higher in the icefish Chaenocephalus aceratus compared to the red-blooded species Notothenia coriiceps. Transcript levels of GPAT1 were quantified in heart ventricles and liver using qRTPCR. Additionally, GPAT1 cDNA was sequenced in the Antarctic notothenioids, C. aceratus and $N$. coriiceps, and in the sub-Antarctic notothenioid, Eleginops maclovinus, to identify amino acid substitutions that may maintain GPAT1 function at cold temperature. Transcript levels of GPAT1 were higher in liver compared to heart ventricles but were not significantly different between the two species. In contrast, transcripts of GPAT2 were only detected in ventricle where they were 6.6-fold higher in $C$. acertatus compared to $N$. coriiceps. These data suggest GPAT1 may be more important for synthesizing triacylglcerol, whereas GPAT2 may regulate synthesis of phospholipids. GPAT1 amino acid sequences are highly conserved among the three notothenioids with 97.9-98.7\% identity. Four amino acid substitutions within the cytosolic region

Abbreviations: DAG, diacylglycerol; dN/dS, rate of nonsynonymous-to-synonymous amino acid substitutions; EF-1 $\alpha$, elongation factor-1 alpha; G3P, glycerol 3-phosphate; GPAT, glycerol 3-phosphate acyltransferase; HKG, housekeeping gene; LPA, lysophosphatidic acid; LPAAT, lysophosphatidic acid acyltransferase; DNA; NRF-1, nuclear respiratory factor 1; PA, phosphatidic acid; PC, phosphatidylcholine; PE, phosphatidylethanolamine; PI, phosphatidylinositol; PS, phosphatidylserine; PGC-1, peroxisome proliferator-activated receptor gamma coactivator 1-; TAG, triacylglycerol; TBP, TATA-binding protein; 18S, 18S ribosomal RNA 
of Antarctic notothenioid GPAT1 may maintain conformational changes necessary for binding and catalysis at cold temperature.

\subsection{INTRODUCTION}

Antarctic notothenioid fishes are excellent model organisms for studying adaptations to life at cold temperature. Notothenioids are the dominant suborder of fishes in the Southern Ocean, where seawater temperatures have been less than $5^{\circ} \mathrm{C}$ for approximately $12 \mathrm{MY}$ and now range between $-1.86^{\circ} \mathrm{C}$ and $2{ }^{\circ} \mathrm{C}$ (Eastman, 1993). While the majority of notothenioids are endemic to the Southern Ocean, $21 \%$ of the species reside north of the Antarctic Polar Front, offshore of New Zealand, South America, Australia, and northern peri-Antarctic Islands (Eastman, 1993). Antarctic notothenioids possess an array of adaptations to life at cold ${ }^{1}$ temperature including cold stable microtubules and lens crystalline proteins (Detrich and Overton, 1988; Kiss et al., 2004), membranes enriched in polyunsaturated fatty acids (Logue et al., 2000), cold-adapted metabolic enzymes (Fields and Somero, 1998), and high mitochondrial densities in some tissues (Johnston et al., 1998).

Elevations in mitochondrial density offset the depressive effects of cold temperature on the catalytic rate of enzymes and increase the diffusion rate of metabolites by minimizing diffusion distances (Sidell, 1998). Aerobic muscles of the hemoglobinless Channichthyidae family of Antarctic notothenioids have remarkably high mitochondrial densities. In heart ventricles, mitochondria displace between $20 \%$ and $37 \%$ of cell volume and in oxidative pectoral 
adductor muscle, between 39\% and 51\% of fiber volume (Archer and Johnston, 1991; Feller et al., 1985; O'Brien and Sidell, 2000; O'Brien et al., 2003). High mitochondrial densities in icefish oxidative muscles do not enhance aerobic metabolic capacity but rather, likely compensate for the lack of hemoglobin $(\mathrm{Hb})$ and myoglobin $(\mathrm{Mb})$ and enhance oxygen storage and diffusion (Sidell and O'Brien, 2006). The lipid-rich mitochondria in icefish hearts facilitate oxygen diffusion because oxygen is approximately four times more soluble in phospholipids than in water (Battino et al., 1968; Smotkin et al., 1991), and oxygen concentrates within the hydrophobic core of the phospholipid bilayer, increasing oxygen storage capacity (Smotkin et al., 1991). The two most abundant mitochondrial phospholipids, phosphatidylethanolamine (PE) and phosphatidylcholine (PC) are 1.3- to 1.4- fold higher per g mitochondrial protein in hearts of the icefish Chaenocephalus aceratus compared to the red-blooded species Notothenia coriiceps (O'Brien and Mueller, 2010). The overall increase in mitochondrial phospholipids may be attributable to an upregulation in the glycerolipid synthesis pathway, yet it is unknown how the synthesis of phospholipids is integrated into mitochondrial biogenesis in either fish or mammals (O'Brien, 2011). The enzyme glycerol-3-phosphate acyltransferase (GPAT), which catalyzes the initial and rate-limiting step in phospholipid and triacylglycerol (TAG) biosynthesis (Wendel et al., 2009), may regulate mitochondrial phospholipid density and contribute to differences in mitochondrial densities between red- and white-blooded notothenioids. Consistent with this, a recent study from our lab has provided evidence that GPAT may regulate mitochondrial membrane biosynthesis in threespine stickleback in response to cold acclimation (Keenan et al., 2016).

GPAT catalyzes the acylation of glycerol-3-phosphate at the $s n-1$ position, synthesizing lysophosphatidic acid (LPA). LPA acyltransferase (LPAAT) then acylates the $s n-2$ position, 
forming phosphatidic acid (PA), which is then hydrolyzed to diacylglycerol (DAG). PA is the precursor for the synthesis of phosphatidylinositol (PI), phosphatidylglycerol (PG) and cardiolipin (CL), whereas DAG is the precursor for synthesizing TAG, phosphatidylethanolamine (PE), phosphatidylcholine (PC) and phosphatidylserine (PS) (Coleman and Lee, 2004). In vertebrates, there are at least four GPAT isoforms (GPAT1-4) differing in their subcellular location, sensitivity to the sulfhydryl reagent N-ethylmaleimide (NEM), and fatty acyl-CoA preference (Coleman and Lee, 2004; Gimeno and Cao, 2008). GPAT1 and GPAT2 are associated with the outer mitochondrial membrane, and in humans have molecular masses of $94 \mathrm{kDa}$ and $88 \mathrm{kDa}$, respectively (Coleman and Lee, 2004). GPAT3 and GPAT4 are localized to the endoplasmic reticulum (ER) membrane, and in humans have molecular masses of $50 \mathrm{kDa}$ and $52 \mathrm{kDa}$, respectively (Cao et al., 2006; Nagle et al., 2008).

Activity and expression of each isoform varies with tissue type. In most tissues, the activity of microsomal GPAT isoforms accounts for $90 \%$ of the total GPAT activity, but in liver activities of the microsomal and mitochondrial isoforms are nearly equivalent (Coleman and Lee, 2004). Activity of the mitochondrial isoforms is higher in liver and adipose compared to heart, although protein levels are higher in heart (Lewin et al., 2001). This discrepancy is likely due to assaying activity of the enzyme in the presence of NEM, and thus only accounting for activity of GPAT1, which is NEM sensitive (Lewin et al., 2001; Lewin et al., 2004). The functional differences among the four GPAT isoforms remain unclear. Most studies to date have focused on the function of GPAT1, which changes in response to diet in a fashion consistent with its role in mediating the TAG biosynthesis, but GPAT1 null mice also display alterations in phospholipid composition, suggesting a role in phospholipid synthesis as well (Hammond et al., 2002; Lewin et al., 2008). 
We hypothesized that if GPAT1 and GPAT2 are involved in synthesizing mitochondrial membranes, then transcript levels of these genes would be higher in heart ventricles of the icefish $C$. aceratus, with mitochondrial densities of $36.53 \pm 2.08 \%$ compared to the red-blooded species $N$. coriiceps, with mitochondrial densities of $18.18 \pm 0.69 \%$ (O'Brien and Sidell, 2000; Urschel and O'Brien, 2008). We also predicted that mRNA levels of both genes would be higher in liver than heart, consistent with expression levels in mammals. Transcript levels of GPAT1 and GPAT2 were quantified in liver and ventricle tissue of $N$. coriiceps and $C$. aceratus using real-time quantitative PCR. GPAT1 cDNA was sequenced in three notothenioid species: C. aceratus, $N$. coriiceps, and the sub-Antarctic species, Eleginops maclovinus, which inhabits warmer coastal waters around the Falkland Islands and South America and diverged prior to the isolation of Antarctica and formation of the circumpolar current (Eastman, 1993), to provide insight to the evolution of a mitochondrial transmembrane protein in fishes inhabiting a chronically cold environment.

\subsection{MATERIAL AND METHODS}

\subsubsection{Tissue Collection}

Notothenia coriiceps (Richardson, 1844) and Chaenocephalus aceratus (Lönnberg, 1905) were captured in Dallmann Bay $\left(64^{\circ} 10^{\prime} \mathrm{S}, 62^{\circ} 35^{\prime} \mathrm{W}\right)$ and off the southwestern shore of Low Island $\left(63^{\circ} 24^{\prime} \mathrm{S}, 62^{\circ} 10^{\prime} \mathrm{W}\right)$ using an otter trawl and baited pots deployed from the ARSV Laurence M. Gould. Animals were maintained in circulating seawater tanks on board the ship and then transferred to circulating seawater tanks at the U.S. Antarctic Research Station, Palmer Station where they were maintained at $0 \pm 0.5^{\circ} \mathrm{C}$. Fish were euthanized by either a sharp blow 
to the head or an overdose of tricaine methanesulfonate (MS-222) (1:7500 in seawater) followed by spinal cord transection. Heart ventricles and livers were excised immediately, frozen in liquid nitrogen, and stored at $-80^{\circ} \mathrm{C}$. Heart ventricles of the sub-Antarctic notothenioid species, $E$. maclovinus (Cuvier), captured in 2004, were provided by Drs. B. Sidell and C. Cheng. Animals used for measuring mRNA abundance were collected in austral fall and winter of 2013 and 2015. Animals used for sequencing GPAT1 cDNA were collected in austral fall and winter of 2007 and 2009. All procedures were approved by the University of Alaska Fairbanks Institutional Animal Care Committee (134774-8).

\subsubsection{RNA isolation and cDNA synthesis}

Total RNA was extracted using the RNeasy Fibrous Tissue Mini Kit (Qiagen, Valencia, CA, USA) following the manufacturer's protocol except that RNA was treated with DNase I twice, once for 25 minutes and second time for 20 minutes. Concentration and purity of RNA were determined spectrophotometrically with a Nanodrop ND-1000 spectrophotometer (ThermoScientific Fisher, Pittsburgh, PA, USA). All samples had 260 nm-to-230 nm ratios of 1.8 - 2.3 and $260 \mathrm{~nm}$-to-280 $\mathrm{nm}$ ratios of 2.0 - 2.2. Integrity of RNA was determined by separating RNA on a $2 \%$ agarose gel stained with ethidium bromide. RNA was stored at $-80^{\circ} \mathrm{C}$ until further use. First-strand complimentary DNA (cDNA) was synthesized using Taqman reverse transcription reagents (Applied Biosystems, Foster City, CA, USA). Each $50 \mu 1$ reaction volume contained $1 \mathrm{mg}$ RNA, 1 X RT buffer, $3 \mathrm{mM} \mathrm{MgCl}_{2}, 2 \mathrm{mM}$ dNTPs, $2.5 \mu \mathrm{M}$ random hexamers, $20 \mathrm{U}$ RNase inhibitor, and $37.5 \mathrm{U}$ reverse transcriptase. cDNA was stored at $-20^{\circ} \mathrm{C}$.

\subsubsection{Sequencing GPAT1 and GPAT2 cDNA}


Degenerate primers were designed using CODEHOP

(http://blocks.fhcrc.org/codehop.html) and based on amino acid sequences from four fish species for GPAT1 (Gasterosteus aculeatus, Ensembl accession no. ENSGACP00000008124;

Dicentrarchus labrax, GenBank accession no. CBN81479.1; Oreochromis niloticus, GenBank accession no. XP 005471367.1; and Takifugu rubripes, GenBank accession no. $\underline{\text { XP }}$ $\underline{\mathbf{0 1 1 6 2 0 1 8 7 . 1}})$ and three species for GPAT2 (G. aculeatus, Ensembl accession no. ENSGACT00000007807.1; T. rubripes, GenBank accession no. XP 011614214.1; O. niloticus, GenBank accession no. XPP 003451146.2 $)$ (Table 1). These primers were used to amplify 502 bp of GPAT1 and $1250 \mathrm{bp}$ of GPAT2 with PCR in a reaction mixture containing $3 \mu 1$ of cDNA, $1 \mathrm{X}$ PCR buffer, $2 \mathrm{mM} \mathrm{MgCl} 2,0.4 \mathrm{mM}$ dNTP's, $1 \mu \mathrm{M}$ forward and reverse primers, and 2.5U Taq polymerase (Promega, Madison, WI, USA) and using a touchdown protocol with an iCycler (Bio-Rad Laboratories, Hercules, CA, USA). A forward gene-specific primer (GSP) for GPAT1 was then designed based on the GPAT1 sequence in $N$. coriiceps and paired with a degenerate reverse primer, designed as described above, to amplify a 1250 bp region using the PCR conditions described above (Table 1). 5' and 3' cDNA ends of GPAT1 were amplified using the SMARTer cDNA Amplification kit (Clontech, Mountain View, CA, USA). GPAT1 in E. maclovinus was sequenced with gene-specific primers (Table 1). PCR products were separated on $2 \%$ agarose gels stained with ethidium bromide. Products of expected length were excised, isolated using a QIAquick Gel Extraction Kit (Qiagen, Valencia, CA, USA), and cloned into Escherichia coli using the pCR 2.1®-TOPO vector and TOP10 One Shot ${ }^{\circledR}$ chemically competent cells (Invitrogen, Carlsbad, CA, USA). Plasmids were isolated using the QIAprep Spin Miniprep Kit (Qiagen), and used as templates for Sanger sequencing reactions using the BigDye Terminator v.3.1 Cycle Sequencing Kit (Applied Biosystems). Products were purified 
with Centri-Sep columns packed with Sephadex G-50 gel (Sigma-Aldrich, St. Louis, MO, USA) and sequenced with an ABI PRISM 3100 (Applied Biosystems). GPAT1 cDNA was sequenced in two individuals from each species. Sequence identity was determined by searching the NCBI nucleotide database (http://blast.ncbi.nlm.nih.gov) for homologous sequences.

dN/dS Analysis

GPAT1 cDNA or deduced transcript sequences from $N$. coriiceps (GenBank accession no.

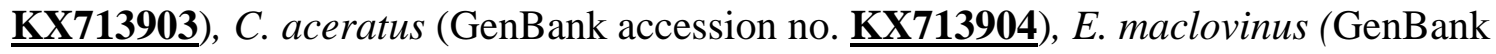
accession no. $\underline{\text { KX713905) }}$, G. aculeatus (Ensembl accession no. ENSGACT00000008124.1), D. rerio (GenBank accession no. XM_009306794.2), Gadus morhua (Ensembl accession no.

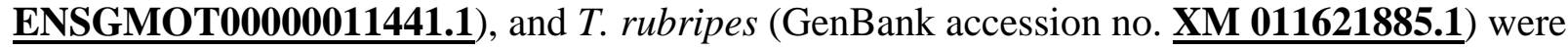
aligned using the ClustalW (codons) algorithm, and a species tree was developed using the Maximum Likelihood method based on the Tamura-Nei model (Tamura and Nei, 1993) in MEGA version 7.0 (Kumar et al., 2016). Alignments were analyzed for rates of nonsynonymous $(\mathrm{dN})$ and synonymous (dS) substitutions to assess the strength and direction of selection using Codeml in the Phylogenetic Analysis by Maximum Likelihood (PAML), version 4.9. The dNto-dS ratios were calculated following Shin et al. (2014) using the basic model (seqtype $=1$, CodonFreq $=2$, model $=0$, NSsites $=0$, fix_omega $=0)$ and the branch model where $($ seqtype $=$ 1, CodonFreq $=2$, model $=2$, NSsites $=0$, fix_omega $=0$ ).

\subsubsection{GPAT1 and GPAT2 transcript levels}

Conserved regions of the full-length GPAT1 cDNA sequences and partial GPAT2 cDNA sequences from $N$. coriiceps and C. aceratus were used to design gene-specific primers (GSP) using Primer Express version 2.0 (Applied Biosystems) (Table 2). Either the forward or reverse 
primer was designed over a splice site to ensure genomic DNA was not amplified. Quantitative real-time PCR (qRT-PCR) was carried out using an ABI PRISM 7900HT (Applied Biosystems). The final reaction mixture $(20 \mu 1)$ contained $10 \mu$ l Power SYBR Green PCR Master Mix (Applied Biosystems), $300 \mathrm{nM}$ forward and reverse primers, and $5 \mathrm{ng}$ of cDNA. Optimum primer concentration was determined by measuring GPAT1 mRNA levels using primer concentrations of 200, 300, and $400 \mathrm{nM}$. Target genes were measured in triplicate in 5-6 individuals per tissue and species. Transcript levels of GPAT1 and GPAT2 genes were normalized to transcript levels of TATA box binding protein (TBP) using the comparative critical threshold ( $\Delta \Delta \mathrm{Ct}$ ) method (Livak and Schmittgen, 2001) and expressed relative to levels in $N$. coriiceps ventricle. Two controls were run to ensure that contaminating or genomic DNA was not amplified, one lacking cDNA and the other containing cDNA synthesized without reverse transcriptase. Melting curve analysis was conducted to verify that only one product was amplified with each primer set.

\subsubsection{Housekeeping gene $(H K G)$ analysis}

Transcript levels of 18S ribosomal RNA (18S), elongation factor 1-A (EF-1 $\alpha$ ), and TATA box binding protein (TBP) were measured in ventricle and liver tissue of 6 individuals of N. coriiceps and C. aceratus using primers described previously (Urschel and O'Brien, 2008) and qRT-PCR as described above except that $1 \mathrm{ng}$ of cDNA was used as a template in reactions to amplify 18S. Results were analyzed using the Excel-based software tool BestKeeper (Pfaffl et al., 2004). BestKeeper does not account for differences in gene expression due to differences in experimental conditions; therefore an ANOVA was also performed to identify significant differences in HKG expression between species and tissues. 


\subsubsection{Statistical analysis}

All statistical analyses were conducted with SigmaPlot 11.0 with significance set at $P<$

0.05. Results are presented as mean \pm s.e.m. A two-way ANOVA followed by a post-hoc Tukey's honestly significant difference (HSD) test was used to determine significant differences in the expression of mRNA levels of GPAT1 in ventricle and liver of $N$. coriiceps and $C$.

aceratus. A Student's t-test was used to determine significant differences in GPAT2 expression in heart ventricles between $C$. aceratus and $N$. coriiceps. Critical threshold $(\mathrm{Ct})$ values were $\log$ transformed to meet assumptions of equal variance and normality, as determined by Levene's Test for Homogeneity of Variance and a Shapiro-Wilk test.

\subsection{RESULTS}

\subsubsection{GPAT1 and GPAT2 transcript levels}

TBP was determined to be the most suitable HKG using BestKeeper (Pfaffl et al., 2004). Transcript levels of all HKGs measured (18S, EF-1 $\alpha$, TBP) had standard deviations $<1.0$ and were significantly correlated with the Bestkeeper index, with the exception of $18 \mathrm{~S}$ in liver. A two-way ANOVA determined there were significant differences in $18 \mathrm{~S}$ and EF1- $\alpha$ between tissues and species $(P<0.05)$ and therefore these two genes were eliminated, and transcript levels of GPAT1 and GPAT2 were normalized to TBP.

GPAT1 mRNA levels were higher in liver than heart ventricle $(\mathrm{F}=13.401, P=0.002)$ but did not differ between species $(\mathrm{F}=3.008, P=0.098)$, nor was there an effect of an interaction between species and tissue ( $\mathrm{F}=0.0128, P=0.911$; Fig. 1A). GPAT2 mRNA levels were 6.6-fold 
higher in hearts of $C$. aceratus compared to $N$. coriiceps $(P=0.017$; Fig. 1B). GPAT2 mRNA was not detected in liver of either species.

\subsubsection{GPAT1 cDNA and amino acid sequence}

GPAT1 has an open-reading frame of 2484 base pairs in $N$. coriiceps and 2481 base pairs in $C$. aceratus and E. maclovinus, corresponding to a coding region of 827 amino acids in N. coriiceps and 826 amino acids in C. aceratus and E. maclovinus (Figs. 2 and 3). The predicted amino acid sequence of GPAT1 contains two transmembrane domains and four acyltransferase motifs within the active site that are highly conserved among Antarctic and coldtemperate notothenioids, displaying $100 \%$ identity among the three species (Fig 3). The cDNA sequences of the two Antarctic species share 98.6\% identity, while the two Antarctic and the sub-Antarctic notothenioid E. maclovinus share 96-96.2\% identity. Amino acid sequences are 98.7\% identical between the two Antarctic species, $97.9 \%$ between $C$. aceratus and $E$. maclovinus and $98.3 \%$ between $N$. coriiceps and E. maclovinus. Overall, there are ten amino acids that differ between the two Antarctic species and E. maclovinus; however, comparing GPAT1 amino acid sequences among notothenioids, temperate and tropical fishes, and higher vertebrates indicates that only four substitutions are unique to the two Antarctic notothenioids: Glu2Ala, Ser421Ala, Asp608Glu and Thr647Ala (Fig. 3). Three of the four substitutions in E. maclovinus are identical to those of the temperate species G. aculeatus, and at position 647 the substitution in E. maclovinus is identical to G. aculeatus as well as to the temperate and tropical fishes used for comparisons (Fig. 3). At position 2, E. maclovinus possesses a glutamic acid, similar to some tropical fishes, whereas G. aculeatus possesses an aspartic acid at this position. 
Following Shin et al. (2014), the ratio of the rate of nonsynonymous substitutions to the rate of synonymous substitutions (dN/dS) was determined for GPAT1 from seven fish species (Fig. 4). A ratio of $<1$ indicates negative, or purifying selection, while a ratio $>1$ indicates positive selection. From the basic model, the average dN/dS ratio of GPAT1 from the fish species examined was calculated to be 0.066 . Using the branch model, dN/dS was calculated for GPAT1 from the seven fish species, and ranged from 0.028 to 0.151 with the highest $\mathrm{dN} / \mathrm{dS}$ ratio in the Antarctic notothenioids and the lowest $\mathrm{dN} / \mathrm{dS}$ ratio in the cold-temperate notothenioid, $E$. maclovinus (Fig. 4).

\subsection{DISCUSSION}

The function of the multiple isoforms of GPAT has not yet been clearly elucidated in mammals and only one study to date has analyzed GPAT structure or function in fishes (Keenan et al., 2016). Here we present data suggesting that GPAT1 likely mediates TAG synthesis in notothenioids, as has been shown for mammals, and that GPAT2 may mediate mitochondrial phospholipid biosynthesis. Additionally, we show that GPAT1, a mitochondrial transmembrane

protein, is highly conserved between Antarctic and cold-temperate notothenioids, sharing 97.998.3\% identity. Despite probable differences in mitochondrial membrane composition between Antarctic and cold-temperate notothenioids, the two transmembrane domains in GPAT1 are $100 \%$ conserved. Moreover, only four amino acids within the cytosolic domain of the protein reflect sites of potential cold adaptation in Antarctic notothenioids.

Patterns of expression may reflect differences in function of GPAT1 and GPAT2 
Hearts of the icefish C. aceratus have 2.3-fold higher mitochondrial densities compared to closely related red-blooded notothenioids, and these differences are largely driven by an increase in mitochondrial membrane density, providing an excellent model system for understanding how mitochondrial membrane synthesis is regulated and integrated into mitochondrial biogenesis (O'Brien, 2011; O'Brien and Mueller, 2010; O'Brien and Sidell, 2000). Here we determined that while transcript levels of the putative rate-limiting enzyme in glycerolipid synthesis, GPAT1, were similar between both hearts and livers of C. aceratus and $N$. coriiceps, mRNA levels of GPAT2 were 6.6-fold higher in hearts of C. aceratus compared to $N$. coriiceps, refelcting the higher density of the phospholipids PE and PC in hearts of $C$. aceratus (O'Brien and Mueller, 2010). These results suggest that GPAT2 may regulate the synthesis of mitochondrial phospholipids, although further studies are needed to confirm this. In contrast, GPAT1 mRNA levels were 3.3-fold higher in livers of $N$. coriiceps and $C$. aceratus compared to their heart ventricles, consistent with higher levels of TAG in liver and the role of GPAT1 in regulating TAG storage in mammalian livers, where changes in GPAT1 mRNA levels change in a similar fashion to TAG content (Cao et al., 2006; Lewin et al., 2008; Lewin et al., 2001; Nagle et al., 2008). In contrast, mRNA levels of GPAT2 are not altered in response to diet nor do they change in response to loss of GPAT1 activity, suggesting GPAT2 may be more important for the synthesis of phospholipids than TAGs (Wang et al., 2007).

Mitochondria in icefish hearts are significantly larger compared to those in red-blooded fishes, with 2-fold higher surface-to-volume ratios, suggesting a greater degree of mitochondrial fusion relative to fission (O'Brien and Sidell, 2000; Urschel and O'Brien, 2008). Based on studies in Caenorhabditis elegans, this suggests the high levels of GPAT2 in icefish hearts might contribute to their unique mitochondrial morphology (Ohba et al., 2013). Deletion of the single 
mitochondrial GPAT isoform (mtGPAT) in C. elegans results in altered mitochondrial morphology and fragmentation, whereas deletion of the two microsomal GPATs had no effect on mitochondria. Although the exact mechanism by which mtGPAT alters mitochondrial morphology is unknown, the authors of the study hypothesize that LPA produced by GPAT2 might facilitate fusion (Ohba et al., 2013).

A recent study provides the first linkage between GPAT and mitochondrial biogenesis, further supporting the role of GPAT2 in regulating mitochondrial membrane density in notothenioids (Senoo et al., 2015). In response to overexpression of PGC-1 $\alpha$, the master transcriptional regulator of mitochondrial biogenesis, GPAT1 and GPAT3 mRNA increase in skeletal muscle and are associated with alterations in membrane composition (Senoo et al., 2015). In fish, NRF-1 and PGC-1 $\beta$ rather than PGC- $1 \alpha$ regulate mitochondrial biogenesis (Bremer et al., 2012; LeMoine et al.). Our results suggest that future studies might explore the capacity of NRF-1 and PGC-1 $\beta$ to regulate GPAT isoforms in response to stimuli that induce mitochondrial biogenesis in fish, such as exercise and cold temperature.

\section{GPAT1 sequence is highly conserved}

GPAT1 amino acid sequences are highly conserved with $97.9-98.3 \%$ identity between Antarctic and cold-temperate notothenioids. The $98.7 \%$ identity between the two Antarctic species reflects the divergence of E. maclovinus from the Antarctic notothenioids approximately 22-23 MYA (Eastman, 1993). The higher degree of similarity between E. maclovinus and $N$. coriiceps $(98.3 \%$ identity) reflects their closer phylogenetic relationship compared to $E$. maclovinus and C. aceratus, with $97.9 \%$ identity. Icefishes are predicted to have diverged from the notothenioid lineage between 5.5 and 2 MYA (Bargelloni et al., 2000) . 
The higher dN/dS values for GPAT1 from C. aceratus and N. coriiceps could be indicative of relaxed purifying selection in cold-adapted notothenioids compared to coldtemperate and temperate fishes. Furthermore, the dN/dS ratio for GPAT1 from the coldtemperate notothenioid, E. maclovinus, is similar to the values calculated for other fish species, which could reflect a greater negative selection pressure due to its temperate environment. The $\mathrm{dN} / \mathrm{dS}$ ratios calculated from GPAT1 were lower than the $\mathrm{dN} / \mathrm{dS}$ ratios calculated for mitochondrial proteins (Shin et al., 2014), with the exception of GPAT1 dN/dS from D. rerio (Fig. 4), which may indicate GPAT1 has greater purifying selection acting on it than other mitochondrial proteins involved with oxidative phosphorylation.

GPAT1 is localized to the outer mitochondrial membrane with both the $\mathrm{N}$ - and C-termini facing the cytosol, two transmembrane domains, an active site between residues 224-323 that faces the cytosol, and a loop within the intermembrane space (Gonzalez-Baro et al., 2001). The four 1-acyl-sn-glycerol-3-phosphate acyltransferase (AGPAT) motifs within the cytosolic region of the protein involved in binding G-3-P and catalysis (Takeuchi and Reue, 2009) are 100\% conserved among the three notothenioids. This is consistent with other studies of cold-adapted proteins, showing that few amino acid substitutions are necessary to maintain catalytic activity at cold temperature, and that these sites typically lie outside of the active site, in areas of the protein that enhance flexibility (Feller and Gerday, 1997; Fields and Somero, 1998; Somero, 2004).

There are four amino acid substitutions in GPAT1 that differ between the two Antarctic notothenioids and cold-temperate notothenioid E. maclovinus and for which the substitution in $E$. maclovinus is similar to that of other temperate fishes, suggesting these substitutions may be important for maintaining enzyme activity in the cold. All four residues lie within the cytosolic 
region of the protein, with one near the $\mathrm{N}$-terminus and three near the $\mathrm{C}$-terminus. These include: Glu2Ala, Ser421Ala, Asp608Glu and Thr647Ala. Two of the four substitutions involve a change from a polar residue in the cold temperate species to a nonpolar one in the Antarctic species- a change characteristic of cold-adapted proteins (Feller and Gerday, 1997; Hochachka and Somero, 2002). An increase in nonpolar residues reduces hydrogen bonding, thereby decreasing stability. Additionally, the substitution of Thr647Ala will enhance protein flexibility because branched amino acids such as Thr restrict the number of potential conformations of the polypeptide backbone, increasing rigidity and stability of the protein (Feller and Gerday, 1997) . While the active site of GPAT1 is located at the N-terminus within the cytosolic region of the protein, deletion analysis indicates that the C-terminus, where three of these substitutions are located, interacts with the N-terminus and is required for enzyme activity (Pellon-Maison et al., 2006). The authors of this study suggest that the C-terminus may contain amino acids necessary for catalysis, or may confer stability necessary for catalysis. Interestingly, GPAT1 sequence in notothenioids, as well as in other fishes examined, have a five amino acid insert within this Cterminal domain (597/598-600/601). Insertions are another strategy employed by cold-adapted organisms to increase protein flexibility (Feller and Gerday, 1997). For example, the peptide transporter, PEPT1, in the icefish Chionodraco hamatus, has between one and six repeats of a seven amino acid sequence at the C-terminus that confers function at cold temperature when transferred to a mammalian PEPT1 (Rizzello et al., 2013). Three other Antarctic teleosts sequenced also had a single insert (Rizzello et al., 2013).

We anticipated that the transmembrane domains of GPAT1 would differ between the cold-temperate and Antarctic notothenioids, reflecting differences in membrane phospholipid composition. Although no studies to date have compared the composition of mitochondrial 
membranes between cold-temperate and Antarctic notothenioids, the most comprehensive study to date on teleost membrane composition in synaptosomes of fishes inhabiting temperate, tropical and polar regions, shows that membrane fluidity is maintained at cold temperature by increasing the proportion of unsaturated fatty acids (Logue et al., 2000). Thus, it is reasonable to assume that Antarctic notothenioids inhabiting waters $6-12^{\circ} \mathrm{C}$ colder than E. maclovinus, would have membranes more enriched in polyunsaturated fatty acids (PUFAs) that might affect protein stability, and yet, both transmembrane domains in GPAT1 are 100\% conserved between Antarctic and cold-temperate species. This is in contrast to the enzyme, $\Delta 9$ desaturase, in which the first transmembrane domain of $\Delta 9$ desaturase of the Antarctic fishes Trematomus bernacchii and Chionodraco hamatus contains two cysteine residues that are absent in the cold-temperate notothenioid, Patagonotothen tessellata, that escaped the polar front, and E. maclovinus contains one of the two cysteine residues (Porta et al., 2013). Protein modeling suggests that the structure of the cold-adapted protein with two cysteine residues forms a more compact structure that may facilitate its insertion into the PUFA-rich membranes of Antarctic notothenioids (Porta et al., 2013).

\subsection{ACKNOWLEDGEMENTS}

We thank the Masters and Crew of the ARSV Laurence M. Gould and the Raytheon support staff at the US Antarctic Research Station, Palmer Station. Funding for this work was provided by a grant from the National Science Foundation (ANT-0741301 to KMO). 


\section{FIGURE LEGENDS}

Figure 1. GPAT1 (A) and GPAT2 (B) mRNA levels in heart ventricle and liver of notothenioids normalized to TBP and expressed relative to levels in heart ventricles of $N$. coriiceps. Significant differences are indicated by an asterisk $(P<0.05 ; n=5-6)$.

Figure 2. cDNA sequence of GPAT1 in three notothenioids species

Figure 3. Amino acid sequence alignment of fish, bird, and mammalian GPAT1. Identical residues are shaded, acyltransferase site motifs I-IV are boxed in black, transmembrane domains I and II are underlined, and substitutions unique to Antarctic notothenioids are shown in white lettering on a black background.

Figure 4. Phylogeny of teleosts with dN/dS of GPAT1 and average dN/dS of mitochondrial proteins, noted with an asterisk, are from Shin et al. (2014). 
Table 1. Degenerate and gene-specific primers used to sequence GPAT1 and GPAT2 cDNA

\begin{tabular}{lll} 
Primer name & Type & Nucleotide sequence (5'-3') \\
\hline GPAT1 F & Degenerate & GCCTGCGGAACGTGATHTAYATHAA \\
GPAT1 R & Degenerate & TGAGGGGCCTTGATGTTRTGRCARAA \\
GPAT1 F2 & Specific & TCGACTACTTGCTCATCACGTT \\
GPAT1 R2 & Degenerate & TCACGGCGTCGTGGAANACYTGRTA \\
GPAT2 F & Degenerate & GGGCCAGTGCTGCCAYCARTGYAC \\
GPAT2 R & Degenerate & CGGTGCCGGGTGGAYGANTGGYT \\
GPAT1 5' RACE & Specific & GAGGAGTTGTTGCGTCAGCAGCAGTTTC \\
GPAT1 3' RACE & Specific & AGCGCTAACCGCAACGGAGAGTTC \\
GPAT1 Emac F & Specific & CAGTCAGGAGAGACTCATCAGAAAGG \\
GPAT1 Emac R & Specific & CTGGGATTGTTTAGAAGTAACCAGTA \\
GPAT Emac F1 & Specific & CTTTTGCAGGGAGACACGGG \\
GPAT Emac R1 & Specific & TGACGTCCCTCTCCATGACA \\
GPAT Emac F2 & Specific & CACCACAGAGCTGGGAGAAG \\
GPAT Emac F3 & Specific & CCTTCCTGTCCACAAGTCCC \\
GPAT Emac R3 & Specific & AACTCGAACGCAACCGTAGT \\
GPAT Emac F4 & Specific & TGTGGGGAATTGCATGTGGA \\
GPAT Emac R4 & Specific & CGAAAAGCGCTGGTACAGTC \\
GPAT Emac R5 & Specific & AATAGTCTGACAGGGTGGTGC \\
GPAT Emac F6 & Specific & CTGAGGATGTTGTCGTGCGT \\
GPAT Emac R6 & Specific & CACAGCAGGGTGAAACCCAAG \\
\hline Findicates forward, R & Cicates reverGe &
\end{tabular}

$\mathrm{F}$ indicates forward, $\mathrm{R}$ indicates reverse primers.

Degenerate nucleotides are indicated as $\mathrm{H}, \mathrm{N}, \mathrm{R}$, and $\mathrm{Y}(\mathrm{H}=\mathrm{A}, \mathrm{C}, \mathrm{T} ; \mathrm{N}=\mathrm{A}, \mathrm{C}, \mathrm{G}, \mathrm{T} ; \mathrm{R}=\mathrm{A}, \mathrm{G} ; \mathrm{Y}=\mathrm{C}, \mathrm{T})$ 
Table 2

Gene-specific primers used to quantify relative transcript levels.

\begin{tabular}{|c|c|}
\hline Primer name & Nucleotide sequence \\
\hline \multirow[t]{2}{*}{ GPAT1 } & F 5' AGAGCCCCTTTCCTGGAGAA 3' \\
\hline & R 5' TCTGCGGAAACGCTCACC 3' \\
\hline \multirow[t]{2}{*}{ GPAT2 } & F 5' GGCTTCCACAACCTGCTGAG 3' \\
\hline & R 5' GCCTCGATACCTGGTGTGTGT 3' \\
\hline \multirow[t]{2}{*}{ TBP } & F 5' CGGGAGCCAAGAGTGAGGA 3' \\
\hline & R 5' GAGCGTATTTTCTGGCAGCTAAC 3' \\
\hline \multirow[t]{2}{*}{$\mathrm{EF} 1-\alpha$} & F 5' CTGGAAGCCAGTGAAAAGATGAC 3' \\
\hline & R 5' ACCACATCCAAGGAAGGCAG 3' \\
\hline \multirow[t]{2}{*}{$18 \mathrm{~S}$} & F 5' ACCACATCCAAGGAAGGCAG 3' \\
\hline & R 5' CCGAGTCGGGAGTGGGTAAT 3' \\
\hline
\end{tabular}

F indicates forward, $\mathrm{R}$ indicates reverse 


\subsection{REFERENCES}

Archer, S.D., Johnston, I.A., 1991. Density of cristae and distribution of mitochondria in the slow muscle fibres of Antarctic fish. Physiol. Zool. 64, 242-258.

Bargelloni, L., Marcato, S., Zane, L., Patarnello, T., 2000. Mitochondrial phylogeny of notothenioids: a molecular approach to Antarctic fish evolution and biogeography. Syst Biol 49, 114-129.

Battino, R., Evans, F.D., Danforth, W.F., 1968. The solubilities of seven gases in olive oil with reference to theories of transport through the cell membrane. J Am Oil Chem Soc 45, 830-833.

Bremer, K., Monk, C.T., Gurd, B.J., Moyes, C.D., 2012. Transcriptional regulation of temperature-induced remodeling of muscle bioenergetics in goldfish. Am J Physiol Regul Integr Comp Physiol 303, R150-158.

Cao, J., Li, J.L., Li, D., Tobin, J.F., Gimeno, R.E., 2006. Molecular identification of microsomal acyl-CoA:glycerol-3-phosphate acyltransferase, a key enzyme in de novo triacylglycerol synthesis. Proc Natl Acad Sci U S A 103, 19695-19700.

Coleman, R.A., Lee, D.P., 2004. Enzymes of triacylglycerol synthesis and their regulation. Prog Lipid Res 43, 134-176.

Detrich, H.W., 3rd, Overton, S.A., 1988. Antarctic fish tubulins: heterogeneity, structure, amino acid compositions and charge. Comparative biochemistry and physiology. B, Comparative biochemistry 90, 593-600.

Eastman, J.T., 1993. Antarctic Fish Biology: Evolution in a Unique Environment. Academic Press, San Diego.

Feller, G., Gerday, C., 1997. Psychrophilic enzymes: molecular basis of cold adaptation. Cell Mol Life Sci 53, 830-841.

Feller, G., Goessens, G., Gerday, C., Bassleer, R., 1985. Heart structure and ventricular ultrastructure of hemoglobin- and myoglobin-free icefish Channichthys rhinoceratus. Cell Tissue Res 242, 669-676.

Fields, P.A., Somero, G.N., 1998. Hot spots in cold adaptation: localized increases in conformational flexibility in lactate dehydrogenase A4 orthologs of Antarctic notothenioid fishes. Proc Natl Acad Sci U S A 95, 11476-11481.

Gimeno, R.E., Cao, J., 2008. Thematic review series: glycerolipids. Mammalian glycerol-3phosphate acyltransferases: new genes for an old activity. J Lipid Res 49, 2079-2088.

Gonzalez-Baro, M.R., Granger, D.A., Coleman, R.A., 2001. Mitochondrial glycerol phosphate acyltransferase contains two transmembrane domains with the active site in the $\mathrm{N}$-terminal domain facing the cytosol. J Biol Chem 276, 43182-43188.

Hammond, L.E., Gallagher, P.A., Wang, S., Hiller, S., Kluckman, K.D., Posey-Marcos, E.L., Maeda, N., Coleman, R.A., 2002. Mitochondrial glycerol-3-phosphate acyltransferase-deficient mice have reduced weight and liver triacylglycerol content and altered glycerolipid fatty acid composition. Molecular and cellular biology 22, 8204-8214.

Hochachka, P.W., Somero, G.N., 2002. Biochemical Adaptation: Mechanism and process in physiological evolution. Oxford University Press, Oxford.

Johnston, I.I., Calvo, J., Guderley, Y.H., 1998. Latitudinal variation in the abundance and oxidative capacities of muscle mitochondria in perciform fishes. J Exp Biol 201 (Pt 1), 1-12. 
Keenan, K., Hoffman, M., Dullen, K., O'Brien, K.M., 2016. Molecular drivers of mitochondrial membrane proliferation in response to cold acclimation in threespine stickleback. Comp Biochem Physiol A Mol Integr Physiol 203, 109-114.

Kiss, A.J., Mirarefi, A.Y., Ramakrishnan, S., Zukoski, C.F., Devries, A.L., Cheng, C.H., 2004. Cold-stable eye lens crystallins of the Antarctic nototheniid toothfish Dissostichus mawsoni Norman. J Exp Biol 207, 4633-4649.

Kumar, S., Stecher, G., Tamura, K., 2016. MEGA7: Molecular Evolutionary Genetics Analysis Version 7.0 for Bigger Datasets. Mol Biol Evol 33, 1870-1874.

LeMoine, C.M., Lougheed, S.C., Moyes, C.D., Modular evolution of PGC-1alpha in vertebrates. J Mol Evol 70, 492-505.

Lewin, T.M., de Jong, H., Schwerbrock, N.J., Hammond, L.E., Watkins, S.M., Combs, T.P., Coleman, R.A., 2008. Mice deficient in mitochondrial glycerol-3-phosphate acyltransferase-1 have diminished myocardial triacylglycerol accumulation during lipogenic diet and altered phospholipid fatty acid composition. Biochim Biophys Acta 1781, 352-358.

Lewin, T.M., Granger, D.A., Kim, J.H., Coleman, R.A., 2001. Regulation of mitochondrial snglycerol-3-phosphate acyltransferase activity: response to feeding status is unique in various rat tissues and is discordant with protein expression. Arch Biochem Biophys 396, 119-127. Lewin, T.M., Schwerbrock, N.M., Lee, D.P., Coleman, R.A., 2004. Identification of a new glycerol-3-phosphate acyltransferase isoenzyme, mtGPAT2, in mitochondria. J Biol Chem 279, 13488-13495.

Livak, K.J., Schmittgen, T.D., 2001. Analysis of relative gene expression data using real-time quantitative PCR and the 2(-Delta Delta C(T)) Method. Methods 25, 402-408.

Logue, J.A., de Vries, A.L., Fodor, E., Cossins, A.R., 2000. Lipid compositional correlates of temperature-adaptive interspecific differences in membrane physical structure. J Exp Biol 203, 2105-2115.

Nagle, C.A., Vergnes, L., Dejong, H., Wang, S., Lewin, T.M., Reue, K., Coleman, R.A., 2008. Identification of a novel sn-glycerol-3-phosphate acyltransferase isoform, GPAT4, as the enzyme deficient in Agpat6-/- mice. J Lipid Res 49, 823-831.

O'Brien, K.M., 2011. Mitochondrial biogenesis in cold-bodied fishes. J Exp Biol 214, 275-285. O'Brien, K.M., Mueller, I.A., 2010. The unique mitochondrial form and function of Antarctic channichthyid icefishes. Integrative and Comparative Biology 50, 993-1008.

O'Brien, K.M., Sidell, B.D., 2000. The interplay among cardiac ultrastructure, metabolism and the expression of oxygen-binding proteins in Antarctic fishes. J Exp Biol 203, 1287-1297.

O'Brien, K.M., Skilbeck, C., Sidell, B.D., Egginton, S., 2003. Muscle fine structure may maintain the function of oxidative fibres in haemoglobinless Antarctic fishes. J Exp Biol 206, 411-421.

Ohba, Y., Sakuragi, T., Kage-Nakadai, E., Tomioka, N.H., Kono, N., Imae, R., Inoue, A., Aoki, J., Ishihara, N., Inoue, T., Mitani, S., Arai, H., 2013. Mitochondria-type GPAT is required for mitochondrial fusion. EMBO J 32, 1265-1279.

Pellon-Maison, M., Coleman, R.A., Gonzalez-Baro, M.R., 2006. The C-terminal region of mitochondrial glycerol-3-phosphate acyltransferase-1 interacts with the active site region and is required for activity. Arch Biochem Biophys 450, 157-166.

Pfaffl, M.W., Tichopad, A., Prgomet, C., Neuvians, T.P., 2004. Determination of stable housekeeping genes, differentially regulated target genes and sample integrity: BestKeeper-Excel-based tool using pair-wise correlations. Biotechnol Lett 26, 509-515. 
Porta, A., Fortino, V., Armenante, A., Maresca, B., 2013. Cloning and characterization of a Delta9-desaturase gene of the Antarctic fish Chionodraco hamatus and Trematomus bernacchii. J Comp Physiol B 183, 379-392.

Rizzello, A., Romano, A., Kottra, G., Acierno, R., Storelli, C., Verri, T., Daniel, H., Maffia, M., 2013. Protein cold adaptation strategy via a unique seven-amino acid domain in the icefish (Chionodraco hamatus) PEPT1 transporter. Proc Natl Acad Sci U S A 110, 7068-7073. Senoo, N., Miyoshi, N., Goto-Inoue, N., Minami, K., Yoshimura, R., Morita, A., Sawada, N., Matsuda, J., Ogawa, Y., Setou, M., Kamei, Y., Miura, S., 2015. PGC-1alpha-mediated changes in phospholipid profiles of exercise-trained skeletal muscle. J Lipid Res 56, 2286-2296. Shin, S.C., Ahn do, H., Kim, S.J., Pyo, C.W., Lee, H., Kim, M.K., Lee, J., Lee, J.E., Detrich, H.W., Postlethwait, J.H., Edwards, D., Lee, S.G., Lee, J.H., Park, H., 2014. The genome sequence of the Antarctic bullhead notothen reveals evolutionary adaptations to a cold environment. Genome biology 15, 468.

Sidell, B.D., 1998. Intracellular oxygen diffusion: the roles of myoglobin and lipid at cold body temperature. Journal of Experimental Biology 201, 1118-1127.

Sidell, B.D., O'Brien, K.M., 2006. When bad things happen to good fish: the loss of hemoglobin and myoglobin expression in Antarctic icefishes. J Exp Biol 209, 1791-1802.

Smotkin, E.S., Moy, F.T., Plachy, W.Z., 1991. Dioxygen solubility in aqueous phosphatidylcholine dispersions. Biochim Biophys Acta 1061, 33-38.

Somero, G.N., 2004. Adaptation of enzymes to temperature: searching for basic "strategies". Comp Biochem Physiol B Biochem Mol Biol 139, 321-333.

Takeuchi, K., Reue, K., 2009. Biochemistry, physiology, and genetics of GPAT, AGPAT, and lipin enzymes in triglyceride synthesis. Am J Physiol Endocrinol Metab 296, E1 195-1209.

Tamura, K., Nei, M., 1993. Estimation of the number of nucleotide substitutions in the control region of mitochondrial DNA in humans and chimpanzees. Mol Biol Evol 10, 512-526.

Urschel, M.R., O'Brien, K.M., 2008. High mitochondrial densities in the hearts of Antarctic icefishes are maintained by an increase in mitochondrial size rather than mitochondrial biogenesis. J Exp Biol 211, 2638-2646.

Wang, S., Lee, D.P., Gong, N., Schwerbrock, N.M., Mashek, D.G., Gonzalez-Baro, M.R., Stapleton, C., Li, L.O., Lewin, T.M., Coleman, R.A., 2007. Cloning and functional characterization of a novel mitochondrial N-ethylmaleimide-sensitive glycerol-3-phosphate acyltransferase (GPAT2). Arch Biochem Biophys 465, 347-358.

Wendel, A.A., Lewin, T.M., Coleman, R.A., 2009. Glycerol-3-phosphate acyltransferases: rate limiting enzymes of triacylglycerol biosynthesis. Biochim Biophys Acta 1791, 501-506. 

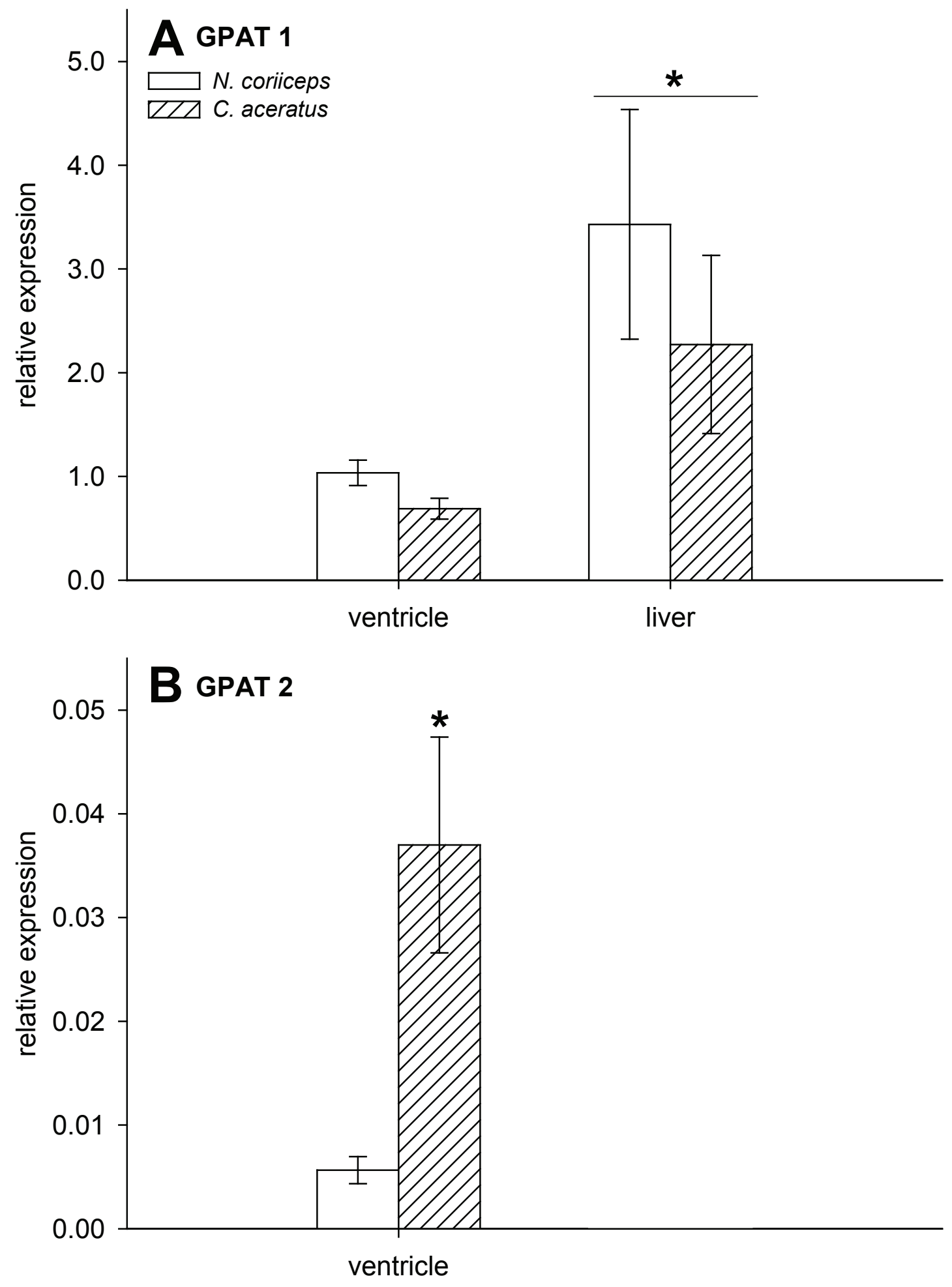


\begin{tabular}{|c|c|c|c|c|c|c|c|c|c|c|c|c|c|c|c|c|c|c|c|c|c|c|c|c|c|c|c|c|c|c|c|c|}
\hline N. coriiceps & $\mathrm{A}$ & $\mathrm{T}$ & $\mathrm{G}$ & $\mathrm{G}$ & $\mathrm{C}$ & $\mathrm{G}$ & $\mathrm{C}$ & $\mathrm{T}$ & G & $\mathrm{T}$ & $\mathrm{C}$ & $\mathrm{A}$ & $\mathrm{G}$ & $\mathrm{A}$ & $\mathrm{C}$ & G & G & $\mathrm{C}$ & $\mathrm{T}$ & $\mathrm{T}$ & $\mathrm{C}$ & T & $T$ & & & & & G & $\mathrm{C}$ & A & $\mathrm{G}$ & 30 \\
\hline C. aceratus & . & . & . & $\cdot$ & . & . & . & . & . & . & . & . & . & . & . & . & . & 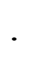 & $\cdot$ & & . & & & & . & & & - & . & . & 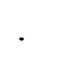 & 30 \\
\hline E. maclovinus & . & $\cdot$ & . & . & A & & & & & . & & . & 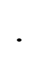 & 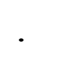 & & & . & & & & & $\mathrm{C}$ & & & & & & & & . & & 30 \\
\hline N. coriiceps & $\mathrm{G}$ & $\mathrm{T}$ & $\mathrm{C}$ & A & $\mathrm{A}$ & $\mathrm{C}$ & A & A & $\mathrm{T}$ & G & $\mathrm{G}$ & A & $\mathrm{G}$ & $\mathrm{A}$ & $\mathrm{G}$ & $\mathrm{C}$ & A & G & $\mathrm{T}$ & $\mathrm{G}$ & $\mathrm{G}$ & A & $\mathrm{C}$ & $\mathrm{T}$ & f & & $A$ & $\mathrm{C}$ & $\mathrm{C}$ & $\mathrm{G}$ & $\mathrm{C}$ & 60 \\
\hline C. aceratus & . & . & . & . & . & . & . & . & . & . & . & . & . & . & . & . & . & . & . & . & . & & . & 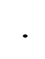 & . & & & . & 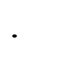 & . & . & 60 \\
\hline E. maclovinus & · & . & . & . & . & 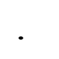 & $\cdot$ & . & . & $\cdot$ & • & . & . & $\cdot$ & - & • & • & & $\cdot$ & & • & & $\cdot$ & G & & & & 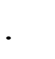 & & . & . & 60 \\
\hline N. coriiceps & $\mathrm{T}$ & $\mathrm{G}$ & G & A & A & $\mathrm{A}$ & $\mathrm{C}$ & A & $\mathrm{T}$ & $\mathrm{C}$ & $\mathrm{C}$ & $\mathrm{C}$ & A & $\mathrm{A}$ & $\mathrm{C}$ & G & A & $\mathrm{T}$ & G & A & $\mathrm{C}$ & $\mathrm{T}$ & $\mathrm{C}$ & G & & i & $A$ & $\mathrm{~T}$ & $\mathrm{C}$ & $\mathrm{G}$ & $\mathrm{C}$ & 0 \\
\hline C. aceratus & . & . & . & . & . & . & . & . & . & . & . & . & . & . & $\cdot$ & . & . & . & . & • & $\cdot$ & • & . & . & • & & & . & . & . & . & 90 \\
\hline E. maclovinus & . & . & . & . & . & . & . & . & . & . & . & . & . & . & . & . & . & . & . & . & . & . & $\cdot$ & . & . & & & . & . & . & $\cdot$ & 90 \\
\hline N. coriiceps & $\mathrm{A}$ & $\mathrm{G}$ & $\mathrm{C}$ & A & $\mathrm{C}$ & $\mathrm{C}$ & A & G & $\mathrm{C}$ & $\mathrm{C}$ & $\mathrm{C}$ & $\mathrm{C}$ & $\mathrm{T}$ & $\mathrm{C}$ & $\mathrm{T}$ & G & $\mathrm{T}$ & $\mathrm{C}$ & $\mathrm{C}$ & $\mathrm{T}$ & $\mathrm{G}$ & $\mathrm{C}$ & $\mathrm{G}$ & $\mathrm{C}$ & 1 & & $\mathrm{G}$ & $\mathrm{C}$ & G & $\mathrm{T}$ & $\mathrm{C}$ & 120 \\
\hline C. aceratus & . & . & . & . & . & . & . & . & . & . & . & . & . & . & . & 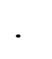 & . & . & . & . & . & . & . & 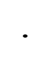 & · & & & . & 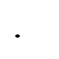 & . & ${ }^{\circ}$ & 120 \\
\hline E. maclovinus & . & . & . & . & . & . & . & . & . & . & 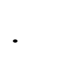 & . & . & . & A & . & $\cdot$ & . & 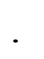 & $\cdot$ & $\cdot$ & ${ }^{\circ}$ & $v^{\circ}$ & $\cdot$ & $\cdot$ & & & . & ${ }^{\circ}$ & . & . & 120 \\
\hline N. coriiceps & $\mathrm{G}$ & $\mathrm{C}$ & $\mathrm{C}$ & A & $\mathrm{G}$ & $\mathrm{C}$ & A & $\mathrm{C}$ & $\mathrm{G}$ & $\mathrm{T}$ & G & G & A & $\mathrm{A}$ & G & G & A & G & G & G & $\mathrm{C}$ & $\mathrm{C}$ & $\mathrm{T}$ & G & ( & 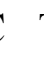 & $\Gamma$ & G & A & $\mathrm{G}$ & $\mathrm{C}$ & 150 \\
\hline C. aceratus & . & . & . & . & . & . & . & . & . & $\cdot$ & . & . & . & . & . & . & . & $\cdot$ & . & $\cdot$ & . & . & . & $\cdot$ & . & & & . & . & . & . & 150 \\
\hline E. maclovinus & . & . & . & . & . & . & . & . & $\cdot$ & . & . & . & $\cdot$ & . & A & . & . & . & $\cdot$ & $\cdot$ & . & $\cdot$ & . & $\cdot$ & . & & & . & . & . & . & 150 \\
\hline N. coriiceps & $\mathrm{A}$ & $\mathrm{G}$ & A & A & $\mathrm{A}$ & $\mathrm{G}$ & A & G & $\mathrm{G}$ & $\mathrm{C}$ & $\mathrm{C}$ & $\mathrm{C}$ & $\mathrm{T}$ & $\mathrm{T}$ & $\mathrm{T}$ & $\mathrm{G}$ & $\mathrm{T}$ & $\mathrm{G}$ & G & G & $\mathrm{C}$ & $\mathrm{C}$ & $\mathrm{G}$ & C & 1 & 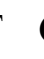 & $\mathrm{G}$ & $\mathrm{C}$ & $\mathrm{T}$ & $\mathrm{G}$ & $\mathrm{T}$ & 18 \\
\hline C. aceratus & . & . & . & . & . & . & . & . & . & . & . & . & . & . & . & . & $\cdot$ & . & . & . & . & $\cdot$ & . & . & . & & & . & . & . & . & 180 \\
\hline E. maclovinus & . & . & . & . & . & . & . & . & . & . & . & . & . & . & . & . & . & . & . & . & . & . & . & . & • & & & . & . & . & . & 180 \\
\hline N. coriiceps & $\mathrm{C}$ & A & $\mathrm{C}$ & $\mathrm{T}$ & $\mathrm{C}$ & $\mathrm{C}$ & $\mathrm{T}$ & G & $\mathrm{C}$ & A & $\mathrm{C}$ & $\mathrm{T}$ & $\mathrm{C}$ & $\mathrm{C}$ & A & $\mathrm{C}$ & A & G & A & G & $\mathrm{C}$ & $\mathrm{T}$ & G & G & & i & A & $\mathrm{G}$ & $\mathrm{A}$ & A & A & 210 \\
\hline C. aceratus & . & . & . & . & . & . & 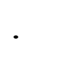 & . & . & . & . & . & . & . & . & . & . & . & . & • & . & . & . & . & . & & & • & . & . & . & 210 \\
\hline E. maclovinus & . & . & $\mathrm{T}$ & 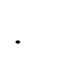 & . & . & . & . & 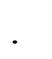 & . & & A & . & . & . & . & . & . & . & & . & & $\cdot$ & & . & & & . & . & . & $\mathrm{G}$ & 210 \\
\hline
\end{tabular}


N. coriiceps

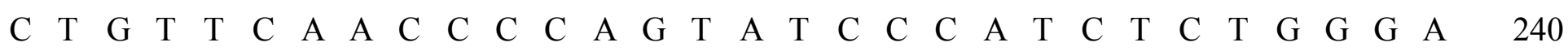

C. aceratus

E. maclovinus • . . . . . . . . . . . . . . . . .

N. coriiceps

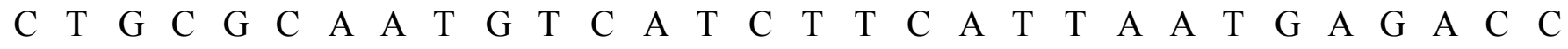

C. aceratus

. . . . . . . . . . . . . . . . . . . . . . . . . . . 270

E. maclovinus

N. coriiceps

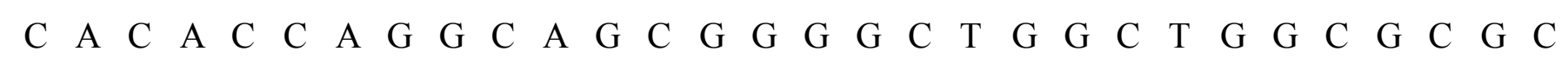

C. aceratus

E. maclovinus

N. coriiceps

C. aceratus

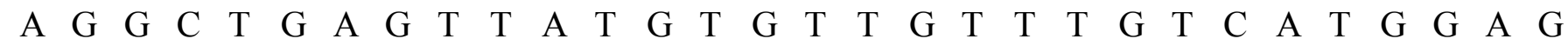

300

E. maclovinus $\mathrm{C}$

N. coriiceps

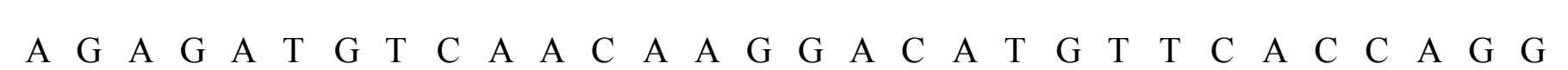

C. aceratus

E. maclovinus

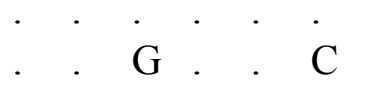

360

N. coriiceps

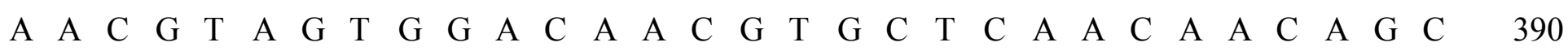

C. aceratus

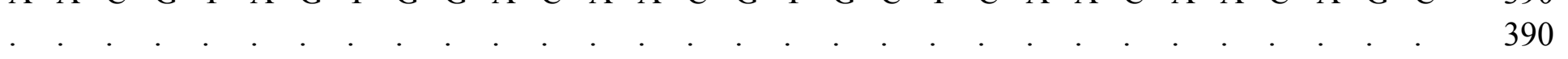

E. maclovinus

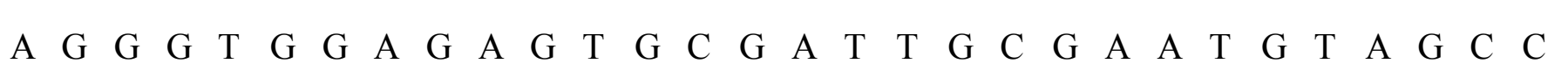

420

N. coriiceps

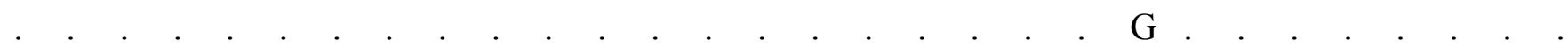

420

E. maclovinus A . 


\begin{tabular}{|c|c|c|c|c|c|c|c|c|c|c|c|c|c|c|c|c|c|c|c|c|c|c|c|c|c|c|c|c|c|c|c|c|}
\hline N. coriiceps & G & $\mathrm{C}$ & $\mathrm{G}$ & G & $\mathrm{A}$ & $\mathrm{T}$ & $\mathrm{G}$ & $\mathrm{T}$ & G & $\mathrm{G}$ & A & $\mathrm{C}$ & $\mathrm{G}$ & $\mathrm{C}$ & $\mathrm{T}$ & $\mathrm{G}$ & $\mathrm{C}$ & A & $\mathrm{G}$ & G & A & $\Delta$ & 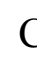 & & & C & $\mathrm{A}$ & $\mathrm{A}$ & $\mathrm{C}$ & $\mathrm{C}$ & $\mathrm{T}$ & 450 \\
\hline C. aceratus & A & . & . & . & . & . & $\mathrm{T}$ & 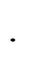 & 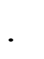 & . & & . & . & . & . & . & . & & . & . & . & & $\cdot$ & & . & & & . & . & . & & 450 \\
\hline E. maclovinus & A & & . & . & . & . & $\mathrm{T}$ & & & . & & . & 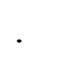 & & & & . & & & & & & & & 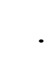 & & & & & . & & 450 \\
\hline N. coriiceps & $\mathrm{G}$ & $\mathrm{G}$ & $\mathrm{C}$ & $\mathrm{C}$ & $\mathrm{A}$ & $\mathrm{G}$ & G & A & G & $\mathrm{C}$ & A & $\mathrm{C}$ & $\mathrm{A}$ & $\mathrm{A}$ & A & $\mathrm{G}$ & $\mathrm{C}$ & $\mathrm{G}$ & G & $\mathrm{T}$ & $\mathrm{C}$ & $\mathrm{A}$ & C & & & $A$ & A & $\mathrm{A}$ & $\mathrm{G}$ & $\mathrm{T}$ & G & 480 \\
\hline C. aceratus & . & . & . & . & . & . & . & . & . & . & . & . & . & . & . & . & . & . & . & & . & $\mathrm{C}$ & & & . & 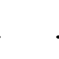 & & . & . & . & & 480 \\
\hline E. maclovinus & & . & . & . & . & . & . & $\cdot$ & $\cdot$ & . & • & . & . & . & . & . & . & . & • & & $\cdot$ & & . & & . & & & $\mathrm{G}$ & & . & & 480 \\
\hline N. coriiceps & $\mathrm{A}$ & A & $\mathrm{G}$ & $\mathrm{C}$ & $\mathrm{A}$ & $\mathrm{G}$ & A & A & A & G & $\mathrm{C}$ & $\mathrm{C}$ & $\mathrm{C}$ & $\mathrm{G}$ & $\mathrm{G}$ & G & $\mathrm{C}$ & $\mathrm{C}$ & $\mathrm{T}$ & $\mathrm{T}$ & $\mathrm{C}$ & $\mathrm{C}$ & $\mathrm{T}$ & 7 & & $\mathrm{C}$ & A & $\mathrm{G}$ & G & $\mathrm{A}$ & G & 510 \\
\hline C. aceratus & . & . & . & . & . & . & . & . & . & . & $\cdot$ & . & . & . & . & . & . & . & $\cdot$ & $\cdot$ & . & . & . & & . & & . & . & ${ }^{\circ}$ & . & . & 510 \\
\hline E. maclovinus & . & . & . & . & . & . & . & . & . & . & . & . & A & . & . & $\mathrm{T}$ & . & . & . & . & . & . & . & & 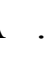 & & & . & . & . & . & 510 \\
\hline N. coriiceps & A & $\mathrm{T}$ & $\mathrm{G}$ & $\mathrm{G}$ & $\mathrm{T}$ & $\mathrm{G}$ & $\mathrm{G}$ & $\mathrm{C}$ & $\mathrm{C}$ & A & A & $\mathrm{C}$ & $\mathrm{A}$ & $\mathrm{T}$ & $\mathrm{T}$ & $\mathrm{T}$ & $\mathrm{C}$ & $\mathrm{A}$ & $\mathrm{C}$ & $\mathrm{C}$ & $\mathrm{G}$ & G & $\mathrm{C}$ & & & T & $\mathrm{T}$ & $\mathrm{C}$ & A & $\mathrm{T}$ & $\mathrm{C}$ & 540 \\
\hline C. aceratus & . & . & . & . & . & . & . & . & . & . & 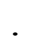 & . & . & . & . & . & . & 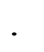 & . & $\cdot$ & . & 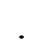 & . & & . & & & . & ${ }^{\circ}$ & . & 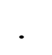 & 540 \\
\hline E. maclovinus & . & . & . & . & . & . & . & . & . & . & . & . & . & . & . & . & . & r. & $\cdot$ & . & $\cdot$ & . & ${ }^{\circ}$ & & . & & $\cdot$ & . & . & . & . & 540 \\
\hline N. coriiceps & $\mathrm{A}$ & $\mathrm{G}$ & G & $\mathrm{C}$ & $\mathrm{T}$ & $\mathrm{G}$ & A & $\mathrm{C}$ & $\mathrm{C}$ & G & G & $\mathrm{C}$ & $\mathrm{T}$ & $\mathrm{G}$ & $\mathrm{G}$ & $\mathrm{G}$ & $\mathrm{T}$ & $\mathrm{C}$ & $\mathrm{C}$ & $\mathrm{T}$ & $\mathrm{C}$ & $\mathrm{C}$ & $\mathrm{T}$ & & & $A$ & $\mathrm{G}$ & G & $\mathrm{C}$ & $\mathrm{T}$ & $\mathrm{C}$ & 570 \\
\hline C. aceratus & . & . & . & . & . & . & . & . & A & . & . & . & . & . & . & . & . & . & . & $\cdot$ & . & $\cdot$ & . & . & . & 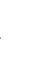 & . & . & . & . & . & 570 \\
\hline E. maclovinus & . & . & . & . & . & . & . & . & A & . & . & . & . & . & . & . & . & - & . & . & . & $\cdot$ & . & $\cdot$ & . & & & . & . & . & . & 570 \\
\hline N. coriiceps & $\mathrm{T}$ & $\mathrm{T}$ & $\mathrm{T}$ & A & A & $\mathrm{T}$ & G & G & $\mathrm{T}$ & $\mathrm{T}$ & $\mathrm{T}$ & $\mathrm{C}$ & $\mathrm{T}$ & $\mathrm{T}$ & $\mathrm{C}$ & $\mathrm{T}$ & G & G & A & G & $\mathrm{C}$ & A & $\mathrm{T}$ & & 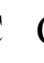 & $\mathrm{C}$ & A & G & $\mathrm{A}$ & $\mathrm{T}$ & $\mathrm{C}$ & 600 \\
\hline C. aceratus & . & . & . & . & . & . & . & . & . & . & . & . & . & . & . & . & - & . & . & . & . & . & . & . & . & . & • & . & . & . & . & 600 \\
\hline E. maclovinus & . & . & . & . & . & . & . & . & . & . & . & . & . & . & . & . & . & . & . & $\cdot$ & . & $\cdot$ & $\cdot$ & 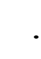 & . & 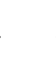 & 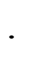 & . & . & . & . & 600 \\
\hline N. coriiceps & $\mathrm{C}$ & A & $\mathrm{C}$ & A & A & $\mathrm{G}$ & G & G & $\mathrm{T}$ & $\mathrm{C}$ & A & G & $\mathrm{C}$ & $\mathrm{T}$ & $\mathrm{G}$ & G & A & A & A & $\mathrm{T}$ & $\mathrm{G}$ & G & $\mathrm{T}$ & & & $A$ & $\mathrm{~A}$ & A & A & A & A & 630 \\
\hline C. aceratus & . & . & . & . & . & . & . & . & . & $\cdot$ & 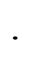 & . & . & . & . & . & . & . & $\cdot$ & . & & $\cdot$ & . & & . & & . & . & . & . & . & 630 \\
\hline E. maclovinus & . & . & . & . & . & . & . & . & . & • & & . & . & . & . & . & . & . &. & $\cdot$ & & . & • & & . & & & . & . & . & & 630 \\
\hline
\end{tabular}


N. coriiceps

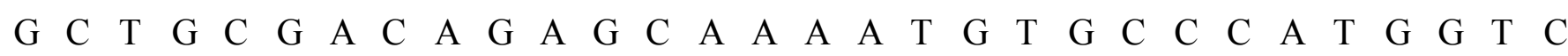

660

C. aceratus

E. maclovinus

660

N. coriiceps

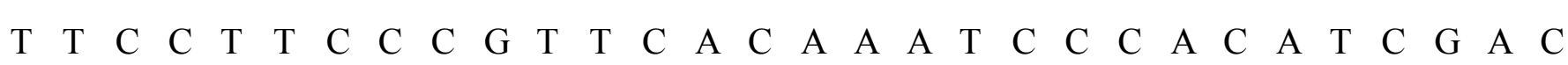

C. aceratus

E. maclovinus

$\cdot \cdot \cdot \cdot \cdot$

N. coriiceps

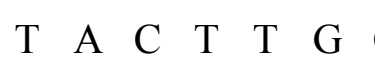

$\mathrm{T}$

C.

690

690

C. aceratus

E. maclovinus

N. coriiceps

C. aceratus

$$
\text { . . . }
$$

T C A $\mathrm{T}$ C A$$
\text { • } \cdot \text {. }
$$$$
\cdot \cdot
$$

C A C A A

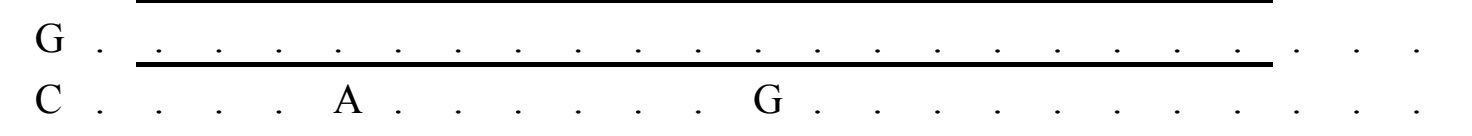

840

C. aceratus

..$~ \cdot \mathrm{G}$ . . 840

E. maclovinus 
$N$. coriiceps

C. aceratus

E. maclovinus

N. coriiceps

C. aceratus

E. maclovinus

$N$. coriiceps

C. aceratus

E. maclovinus

N. coriiceps

C. aceratus

E. maclovinus

$N$. coriiceps

C. aceratus

E. maclovinus

N. coriiceps

C. aceratus

E. maclovinus

N. coriiceps

C. aceratus

E. maclovinus

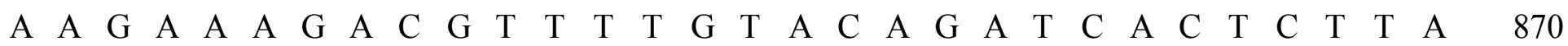

870

870

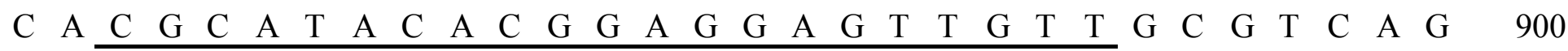

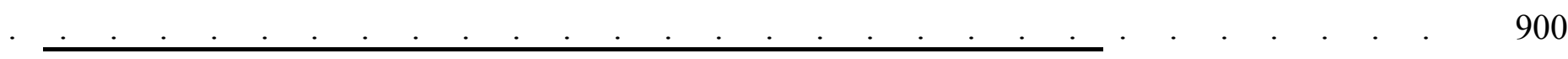

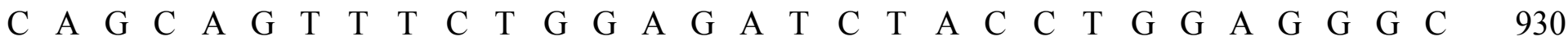

930

930

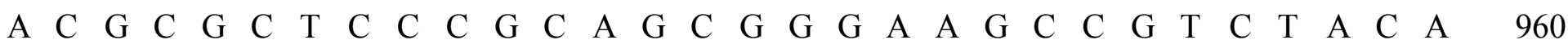

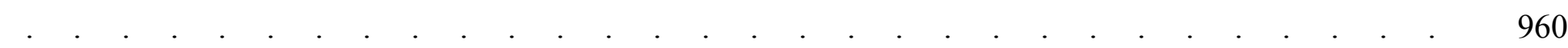

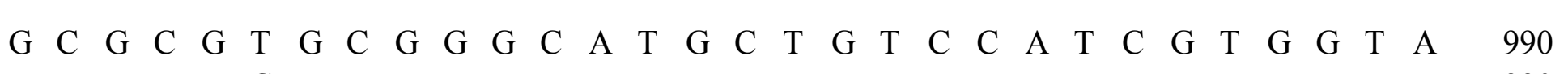

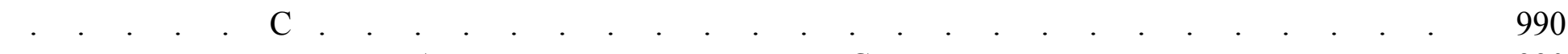

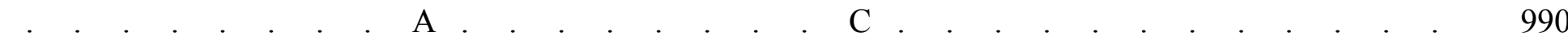

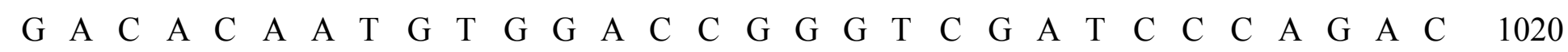

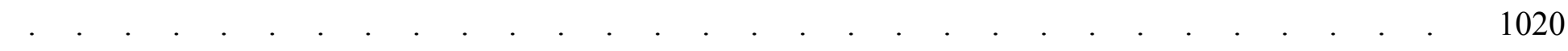

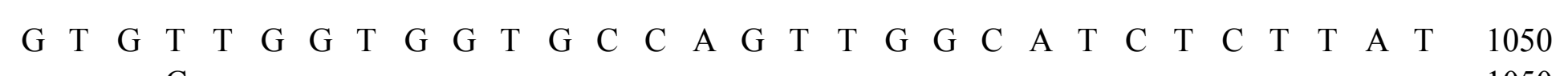

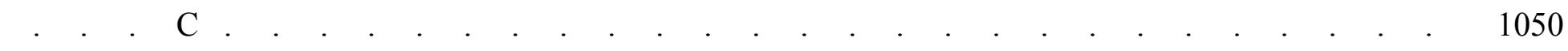

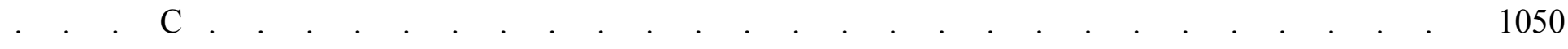




\begin{tabular}{|c|c|c|c|c|c|c|c|c|c|c|c|c|c|c|c|c|c|c|c|c|c|c|c|c|c|c|c|c|c|c|c|}
\hline$N$. coriiceps & $\mathrm{G}$ & A & $\mathrm{T}$ & $\mathrm{C}$ & G & $\mathrm{T}$ & A & $\mathrm{T}$ & $\mathrm{T}$ & $\mathrm{C}$ & $\mathrm{T}$ & $\mathrm{T}$ & $\mathrm{G}$ & $\mathrm{A}$ & $\mathrm{G}$ & $\mathrm{G}$ & $\mathrm{G}$ & $\mathrm{C}$ & A & A & $\mathrm{C}$ & $\mathrm{T}$ & $\mathrm{A}$ & $\mathrm{T}$ & A & $\mathrm{A}$ & $\mathrm{T}$ & A & $\mathrm{G}$ & $\mathrm{C}$ & 1080 \\
\hline C. aceratus & . & . & . & . & $\cdot$ & . & . & $\cdot$ & . & . & $\cdot$ & . & $\cdot$ & . & . & $\bullet$ & . & & $\cdot$ & $\cdot$ & $\cdot$ & & . & . & . & & • & & & & 1080 \\
\hline E. maclovinus & & . & . & . & . & . & $\cdot$ & & & . & & 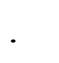 & . & . & $\mathrm{A}$ & & . & & 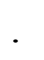 & & 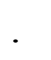 & & . & & & & & & & & 1080 \\
\hline N. coriiceps & $\mathrm{G}$ & A & $\mathrm{G}$ & $\mathrm{C}$ & A & $\mathrm{G}$ & $\mathrm{C}$ & $\mathrm{T}$ & $\mathrm{G}$ & $\mathrm{G}$ & $\mathrm{G}$ & $\mathrm{C}$ & $\mathrm{A}$ & $\mathrm{A}$ & $\mathrm{G}$ & $\mathrm{C}$ & $\mathrm{C}$ & $\mathrm{T}$ & A & A & $\mathrm{G}$ & $\mathrm{A}$ & A & $\mathrm{G}$ & A & A & $\mathrm{T}$ & $\mathrm{G}$ & A & $\mathrm{G}$ & 1110 \\
\hline C. aceratus & . & . & . & . & . & . & . & . & . & . & 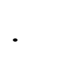 & $\cdot$ & . & . & . & . & . & . & . & . & . & . & . & . & $\cdot$ & & . & & 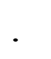 & & 1110 \\
\hline E. maclovinus & . & . & . & . & . & . & . & . & . & . & . & . & . & . & . & . & . & . & $\cdot$ & . & . & . & . & . & $\cdot$ & . & . & . & . & 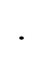 & 1110 \\
\hline N. coriiceps & $\mathrm{A}$ & G & $\mathrm{T}$ & $\mathrm{T}$ & $\mathrm{T}$ & $\mathrm{G}$ & $\mathrm{T}$ & $\mathrm{G}$ & $\mathrm{G}$ & $\mathrm{G}$ & $\mathrm{G}$ & $\mathrm{G}$ & $\mathrm{A}$ & $\mathrm{T}$ & $\mathrm{T}$ & G & $\mathrm{C}$ & $\mathrm{A}$ & $\mathrm{T}$ & $\mathrm{G}$ & $\mathrm{T}$ & G & $\mathrm{G}$ & $\mathrm{A}$ & $\mathrm{G}$ & $\mathrm{T}$ & G & $\mathrm{T}$ & $\mathrm{T}$ & $\mathrm{T}$ & 1140 \\
\hline C. aceratus & . & . & . & . & . & . & . & . & . & . & . & A & . & . & . & . & . & . & $\cdot$ & . & . & & . & . & $\cdot$ & & . & . & . & & 1140 \\
\hline E. maclovinus & . & . & . & . & . & . & . & . & . & . & 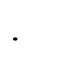 & A & - & . & . & . & . & . & 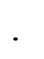 & 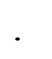 & . & • & . & 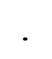 & & & $\cdot$ & & . & $\mathrm{C}$ & 1140 \\
\hline N. coriiceps & $\mathrm{A}$ & G & $\mathrm{G}$ & A & $\mathrm{T}$ & $\mathrm{G}$ & $\mathrm{C}$ & $\mathrm{T}$ & $\mathrm{G}$ & A & $\mathrm{G}$ & $\mathrm{G}$ & $\mathrm{A}$ & $\mathrm{A}$ & $\mathrm{G}$ & A & A & $\mathrm{C}$ & $\mathrm{T}$ & A & $\mathrm{C}$ & $\mathrm{G}$ & $\mathrm{G}$ & $\mathrm{T}$ & $\mathrm{T}$ & G & $\mathrm{T}$ & $\mathrm{G}$ & $\mathrm{T}$ & $\mathrm{T}$ & 1170 \\
\hline C. aceratus & . & . & . & . & . & . & . & . & . & . & . & . & . & . & . & . & . & . & . & . & . & . & . & . & . & 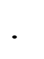 & ${ }^{\circ}$ & $\cdot$ & . & & 1170 \\
\hline E. maclovinus & . & . & . & . & . & . & . & . & . & . & . & . & . & . & . & . & . & . & . & . & . & . & . & . & . & 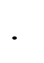 & $\mathrm{C}$ & & . & & 1170 \\
\hline N. coriiceps & $\mathrm{C}$ & G & A & G & $\mathrm{T}$ & $\mathrm{T}$ & G & A & $\mathrm{C}$ & $\mathrm{T}$ & $\mathrm{T}$ & $\mathrm{C}$ & A & $\mathrm{A}$ & $\mathrm{T}$ & $\mathrm{C}$ & A & G & $\mathrm{C}$ & $\mathrm{C}$ & $\mathrm{C}$ & $\mathrm{T}$ & $\mathrm{T}$ & $\mathrm{C}$ & $\mathrm{T}$ & $\mathrm{C}$ & $\mathrm{C}$ & $\mathrm{T}$ & $\mathrm{T}$ & A & 1200 \\
\hline C. aceratus & . & . & . & . & . & . & . & . & . & . & . & 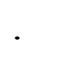 & . & . & . & . & 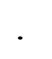 & . & $\cdot$ & . & . & . & . & . & $\cdot$ & $\cdot$ & $\cdot$ & 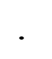 & . & . & 1200 \\
\hline E. maclovinus & . & . & . & . & . & . & . & . & . & . & . & . & . & . & $\mathrm{C}$ & . & . & $\cdot$ & . & . & $\cdot$ & . & $\cdot$ & ${ }^{\circ}$ & & • & . & $\cdot$ & . & . & 1200 \\
\hline N. coriiceps & $\mathrm{A}$ & $\mathrm{A}$ & $\mathrm{G}$ & $\mathrm{G}$ & $\mathrm{A}$ & G & $\mathrm{T}$ & A & $\mathrm{C}$ & $\mathrm{C}$ & $\mathrm{T}$ & $\mathrm{G}$ & $\mathrm{G}$ & $\mathrm{A}$ & $\mathrm{T}$ & $\mathrm{T}$ & $\mathrm{C}$ & $\mathrm{C}$ & $\mathrm{C}$ & A & G & A & G & A & $\mathrm{A}$ & A & $\mathrm{C}$ & $\mathrm{C}$ & G & $\mathrm{C}$ & 1230 \\
\hline C. aceratus & . & . & . & . & . & . & . & . & . & . & . & . & . & . & . & . & . & . & . & $\cdot$ & $\cdot$ & . & . & . & . & . & . & . & $\cdot$ & & 1230 \\
\hline E. maclovinus & . & . & . & . & 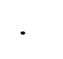 & . & . & . & . & . & . & 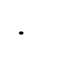 & . & . & . & . & . & . & . & . & . & . & . & . & & 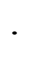 & . & . & . & . & 1230 \\
\hline N. coriiceps & $\mathrm{C}$ & A & $\mathrm{T}$ & $\mathrm{A}$ & $\mathrm{T}$ & $\mathrm{T}$ & $\mathrm{C}$ & $\mathrm{C}$ & $\mathrm{T}$ & $\mathrm{C}$ & $\mathrm{C}$ & $\mathrm{A}$ & $\mathrm{G}$ & $\mathrm{C}$ & $\mathrm{A}$ & G & $\mathrm{T}$ & G & $\mathrm{T}$ & $\mathrm{C}$ & $\mathrm{T}$ & $\mathrm{C}$ & $\mathrm{T}$ & G & $\mathrm{G}$ & A & G & $\mathrm{C}$ & A & $\mathrm{C}$ & 1260 \\
\hline C. aceratus & . & . & . & . & . & . & . & . & . & . & 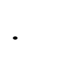 & . & . & . & . & . & . & . & . & . & . & . & . & . & & & . & . & . & A & 1260 \\
\hline E. maclovinus & . & . & . & . & . & . & $\cdot$ & $\cdot$ & & . & & 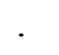 & $\mathrm{T}$ & . & $\cdot$ & & • & & & & 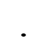 & & & & & & $\cdot$ & & $\cdot$ & & 1260 \\
\hline
\end{tabular}




\begin{tabular}{|c|c|c|c|c|c|c|c|c|c|c|c|c|c|c|c|c|c|c|c|c|c|c|c|c|c|c|c|c|c|c|c|}
\hline N. coriiceps & A & $\mathrm{C}$ & $\mathrm{C}$ & $\mathrm{T}$ & $\mathrm{T}$ & G & A & $\mathrm{T}$ & $\mathrm{G}$ & $\mathrm{C}$ & $\mathrm{C}$ & $\mathrm{C}$ & $\mathrm{A}$ & $\mathrm{T}$ & $\mathrm{C}$ & A & $\mathrm{T}$ & $\mathrm{C}$ & $\mathrm{A}$ & $\mathrm{T}$ & $\mathrm{T}$ & $\mathrm{T}$ & $\mathrm{C}$ & $\mathrm{T}$ & G & $\mathrm{C}$ & A & $\mathrm{C}$ & A & A & 1290 \\
\hline C. aceratus & . & . & . & . & $\cdot$ & . & . & $\cdot$ & . & . & . & $\cdot$ & $\cdot$ & • & . & & . & & $\cdot$ & $\cdot$ & • & & . & $\mathrm{G}$ & & & $\cdot$ & & . & & 1290 \\
\hline E. maclovinus & & . & . & . & . & . & $\cdot$ & & 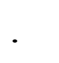 & . & & . & . & & 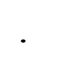 & & . & $\mathrm{T}$ & & & . & & . & 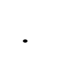 & . & & 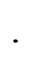 & & . & & 1290 \\
\hline N. coriiceps & $\mathrm{C}$ & $\mathrm{C}$ & $\mathrm{T}$ & $\mathrm{G}$ & $\mathrm{A}$ & $\mathrm{T}$ & $\mathrm{G}$ & $\mathrm{C}$ & $\mathrm{C}$ & $\mathrm{C}$ & $\mathrm{A}$ & G & $\mathrm{C}$ & $\mathrm{T}$ & $\mathrm{G}$ & $\mathrm{T}$ & $\mathrm{T}$ & $\mathrm{T}$ & $\mathrm{G}$ & A & $\mathrm{G}$ & $\mathrm{G}$ & $\mathrm{G}$ & $\mathrm{G}$ & $\mathrm{C}$ & A & $\mathrm{G}$ & $\mathrm{G}$ & A & $\mathrm{G}$ & 1320 \\
\hline C. aceratus & 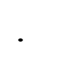 & . & $\mathrm{G}$ & & . & . & . & . & . & . & ${ }^{\circ}$ & . & . & . & . & 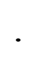 & . & 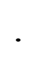 & . & 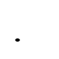 & . & 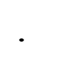 & . & . & . & & . & & & & 1320 \\
\hline E. maclovinus & . & . & . & . & . & . & . & . & $\mathrm{G}$ & . & . & . & . & . & . & . & . & . & . & . & . & . & . & . & . & . & . & . & . & 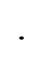 & 1320 \\
\hline N. coriiceps & $\mathrm{G}$ & A & $\mathrm{G}$ & $\mathrm{G}$ & $\mathrm{A}$ & $\mathrm{G}$ & $\mathrm{G}$ & $\mathrm{A}$ & $\mathrm{G}$ & $\mathrm{C}$ & $\mathrm{A}$ & $\mathrm{G}$ & $\mathrm{C}$ & $\mathrm{T}$ & $\mathrm{G}$ & $\mathrm{A}$ & A & $\mathrm{C}$ & $\mathrm{A}$ & $\mathrm{G}$ & $\mathrm{A}$ & $\mathrm{G}$ & A & $\mathrm{G}$ & A & $\mathrm{T}$ & $\mathrm{G}$ & $\mathrm{C}$ & $\mathrm{C}$ & $\mathrm{A}$ & 1350 \\
\hline C. aceratus & . & . & . & . & . & . & $\mathrm{C}$ & . & . & . & . & . & - & - & - & . & • & . & . & . & . & . & . & . & . & & . & . & . & & 1347 \\
\hline E. maclovinus & . & . & . & . & . & . & $\mathrm{C}$ & 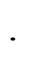 & . & . & $\mathrm{T}$ & 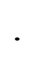 & - & - & - & • & . & & . & . & G & $\cdot$ & 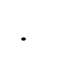 & . & 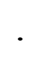 & & • & $\cdot$ & . & & 1347 \\
\hline N. coriiceps & $\mathrm{G}$ & A & $\mathrm{G}$ & $\mathrm{G}$ & $\mathrm{A}$ & $\mathrm{C}$ & A & $\mathrm{T}$ & $\mathrm{C}$ & $\mathrm{G}$ & $\mathrm{T}$ & $\mathrm{G}$ & A & $\mathrm{G}$ & $\mathrm{G}$ & $\mathrm{C}$ & G & $\mathrm{C}$ & $\mathrm{C}$ & A & $\mathrm{A}$ & $\mathrm{C}$ & $\mathrm{T}$ & $\mathrm{T}$ & A & $\mathrm{T}$ & A & A & A & $\mathrm{C}$ & 1380 \\
\hline C. aceratus & . & . & . & . & . & . & . & . & . & . & . & . & . & . & . & . & . & . & . & . & . & 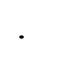 & . & . & . & 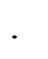 & 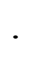 & $\cdot$ & . & & 1377 \\
\hline E. maclovinus & . & . & . & . & . & . & . & . & . & . & 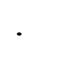 & A & . & . & . & . & . & A & . & . & . & 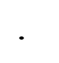 & . & . & . & 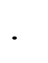 & $\mathrm{C}$ & & . & & 1377 \\
\hline N. coriiceps & A & A & $\mathrm{C}$ & $\mathrm{C}$ & $\mathrm{T}$ & $\mathrm{G}$ & G & $\mathrm{C}$ & $\mathrm{C}$ & A & A & G & $\mathrm{C}$ & $\mathrm{A}$ & $\mathrm{C}$ & G & $\mathrm{T}$ & $\mathrm{C}$ & $\mathrm{C}$ & $\mathrm{T}$ & $\mathrm{C}$ & $\mathrm{T}$ & $\mathrm{T}$ & $\mathrm{C}$ & A & $\mathrm{C}$ & $\mathrm{G}$ & G & $\mathrm{C}$ & $\mathrm{T}$ & 1410 \\
\hline C. aceratus & . & . & . & . & . & . & . & . & . & . & . & . & . & . & . & . & . & . & . & 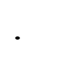 & . & 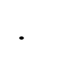 & . & . & . & . & . & . & $\cdot$ & . & 1407 \\
\hline E. maclovinus & . & . & . & . & . & . & . & . & . & . & . & . & . & . & . & · & . & $\cdot$ & . & . & . & . & . & . & . & • & . & $\cdot$ & $\cdot$ & • & 1407 \\
\hline N.coriiceps & A & A & $\mathrm{T}$ & A & A & $\mathrm{G}$ & $\mathrm{T}$ & $\mathrm{C}$ & A & $\mathrm{T}$ & $\mathrm{C}$ & A & G & $\mathrm{C}$ & $\mathrm{G}$ & A & $\mathrm{T}$ & $\mathrm{C}$ & A & $\mathrm{T}$ & $\mathrm{G}$ & $\mathrm{T}$ & $\mathrm{C}$ & $\mathrm{C}$ & A & $\mathrm{C}$ & $\mathrm{C}$ & $\mathrm{C}$ & A & $\mathrm{C}$ & 1440 \\
\hline C.aceratus & . & . & $\cdot$ & . & . & . & . & . & $\cdot$ & . & . & · & . & . & . & . & • & $\cdot$ & . & . & . & . & . & . & $\cdot$ & & $\cdot$ & • & $\cdot$ & & 1437 \\
\hline E.maclovinus & . & . & $\mathrm{C}$ & . & 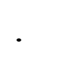 & . & . & . & $\mathrm{C}$ & 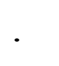 & 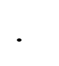 & 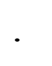 & . & . & . & . & . & & . & 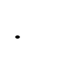 & . & . & . & . & . & & . & . & . & . & 1437 \\
\hline N. coriiceps & A & $\mathrm{T}$ & $\mathrm{T}$ & A & $\mathrm{T}$ & $\mathrm{A}$ & G & $\mathrm{C}$ & $\mathrm{C}$ & $\mathrm{T}$ & G & $\mathrm{T}$ & $\mathrm{C}$ & $\mathrm{T}$ & $\mathrm{C}$ & $\mathrm{C}$ & $\mathrm{T}$ & G & $\mathrm{C}$ & $\mathrm{T}$ & $\mathrm{G}$ & $\mathrm{T}$ & A & $\mathrm{T}$ & A & G & A & $\mathrm{C}$ & A & $\mathrm{C}$ & 1470 \\
\hline C. aceratus & . & . & . & . & . & . & . & . & . & . & . & . & . & . & . & . & . & & . & . & . & . & . & $\mathrm{C}$ & . & & . & . & . & . & 1467 \\
\hline E. maclovinus & . & . & $\mathrm{C}$ & . & . & . & & - & & . & & . & 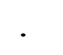 & $\cdot$ & $\cdot$ & & $\cdot$ & & & & . & & $\cdot$ & $\mathrm{C}$ & $\cdot$ & & • & & $\cdot$ & & 1467 \\
\hline
\end{tabular}




\begin{tabular}{|c|c|c|c|c|c|c|c|c|c|c|c|c|c|c|c|c|c|c|c|c|c|c|c|c|c|c|c|c|c|c|c|c|c|}
\hline N. coriiceps & $\mathrm{A}$ & G & $\mathrm{A}$ & $\mathrm{C}$ & $\mathrm{A}$ & $\mathrm{G}$ & $\mathrm{G}$ & $\mathrm{G}$ & $\mathrm{G}$ & G & $\mathrm{T}$ & $\mathrm{G}$ & $\mathrm{G}$ & $\mathrm{T}$ & G & & & & & & $C$ & $C$ & & & ( & & 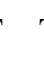 & $\Gamma$ & G & $\mathrm{G}$ & $\mathrm{T}$ & $\mathrm{G}$ & 1500 \\
\hline C. aceratus & . & . & $\cdot$ & $\cdot$ & . & . & . & . & . & . & $\cdot$ & . & . & . & • & & & & & & & . & & & & & & & • & . & . & & 1497 \\
\hline E. maclovinus & 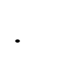 & . & . & . & . & . & 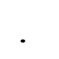 & . & 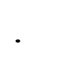 & . & & . & . & $\mathrm{G}$ & & & & & & & & & & & & & & & & & & & 1497 \\
\hline N. coriiceps & $\mathrm{G}$ & A & $\mathrm{A}$ & $\mathrm{G}$ & $\mathrm{A}$ & $\mathrm{C}$ & $\mathrm{T}$ & $\mathrm{T}$ & $\mathrm{C}$ & $\mathrm{T}$ & $\mathrm{T}$ & $\mathrm{C}$ & $\mathrm{A}$ & $\mathrm{A}$ & $\mathrm{C}$ & $A$ & 1 & & J & A & A & $\mathrm{G}$ & $\mathrm{G}$ & $\mathrm{A}$ & ( & & & $A$ & G & A & $\mathrm{T}$ & C & 1530 \\
\hline C. aceratus & . & . & . & . & . & . & . & . & . & . & . & . & . & . & $\cdot$ & & & & & & . & 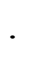 & & & & & & & & 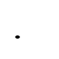 & & & 1527 \\
\hline E. maclovinus & . & . & . & . & . & . & . & . & . & $\cdot$ & 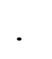 & . & . & . & . & & & & & & & • & & & & & & & • & • & & & 1527 \\
\hline N. coriiceps & $\mathrm{C}$ & $\mathrm{T}$ & G & $\mathrm{T}$ & $\mathrm{C}$ & A & $\mathrm{C}$ & $\mathrm{G}$ & $\mathrm{G}$ & G & A & $\mathrm{C}$ & $\mathrm{T}$ & $\mathrm{T}$ & $\mathrm{T}$ & G & & & & $\mathrm{C}$ & $\mathrm{T}$ & G & G & $\mathrm{G}$ & ( & & 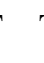 & $\mathrm{T}$ & $\mathrm{C}$ & $\mathrm{T}$ & $\mathrm{C}$ & G & 1560 \\
\hline C. aceratus & . & . & $\mathrm{T}$ & . & . & . & . & . & . & . & $\cdot$ & . & . & . & . & & • & & & & . & . & & & & & & & . & . & . & & 1557 \\
\hline E. maclovinus & . & . & . & . & . & - & A & . & . & . & . & . & . & . & . & & & & & & $\cdot$ & . & & & & & & & $\mathrm{T}$ & & . & & 1557 \\
\hline N. coriiceps & G & $\mathrm{G}$ & G & A & A & $\mathrm{C}$ & $\mathrm{T}$ & $\mathrm{C}$ & $\mathrm{T}$ & G & A & G & G & $\mathrm{A}$ & $\mathrm{T}$ & $\mathrm{G}$ & & & & $\mathrm{G}$ & $\mathrm{T}$ & $\mathrm{G}$ & G & $\mathrm{T}$ & & & & G & $\mathrm{T}$ & G & $\mathrm{C}$ & $\mathrm{C}$ & 1590 \\
\hline C. aceratus & . & . & . & . & . & . & . & . & . & . & ${ }^{\circ}$ & . & . & . & $\cdot$ & & & & & & 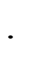 & . & & . & & & & . & . & 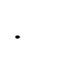 & 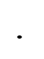 & . & 1587 \\
\hline E. maclovinus & . & . & . & . & . & . & . & . & . & . & . & . & . & . & $\cdot$ & & & & & & . & $\mathrm{C}$ & & & & & & & . & . & $\cdot$ & & 1587 \\
\hline N. coriiceps & $\mathrm{C}$ & $\mathrm{T}$ & $\mathrm{C}$ & $\mathrm{C}$ & $\mathrm{A}$ & $\mathrm{C}$ & $\mathrm{C}$ & $\mathrm{T}$ & $\mathrm{G}$ & $\mathrm{C}$ & $\mathrm{T}$ & G & $\mathrm{G}$ & $\mathrm{G}$ & $\mathrm{A}$ & $A$ & & & $y$ & T & G & $\mathrm{C}$ & $\mathrm{G}$ & $\mathrm{T}$ & C & & & A & $\mathrm{C}$ & G & $\mathrm{T}$ & $\mathrm{G}$ & 1620 \\
\hline C. aceratus & . & . & . & . & $\cdot$ & . & . & . & . & . & . & . & . & . & . & & & & & & • & . & & . & & & & & . & 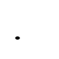 & $\cdot$ & . & 1617 \\
\hline E. maclovinus & . & . & . & . & $\cdot$ & . & . & . & . & $\cdot$ & $\cdot$ & . & . & . & G & & & & & & & . & & . & & & & & . & 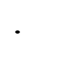 & $\cdot$ & . & 1617 \\
\hline N. coriiceps & A & $\mathrm{C}$ & $\mathrm{C}$ & A & $\mathrm{G}$ & $\mathrm{C}$ & A & $\mathrm{G}$ & $\mathrm{C}$ & G & $\mathrm{C}$ & $\mathrm{T}$ & A & A & $\mathrm{C}$ & C & & & & $A$ & A & $\mathrm{C}$ & $\mathrm{G}$ & G & & & i & $A$ & $\mathrm{G}$ & $\mathrm{T}$ & $\mathrm{T}$ & $\mathrm{C}$ & 1650 \\
\hline C. aceratus & . & . & . & . & . & . & . & . & ${ }^{\circ}$ & . & . & . & . & . & & $\cdot$ & • & & & & . & - & & $\cdot$ & & . & & & . & . & . & . & 1647 \\
\hline E. maclovinus & . & . & . & . & . & . & . & . & $\mathrm{T}$ & . & & A & . & . & & . & & & & & 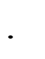 & . & & . & & & & & . & . & . & . & 1647 \\
\hline N. coriiceps & A & $\mathrm{C}$ & $\mathrm{C}$ & A & $\mathrm{T}$ & $\mathrm{C}$ & $\mathrm{G}$ & $\mathrm{C}$ & $\mathrm{G}$ & $\mathrm{C}$ & $\mathrm{C}$ & G & A & $\mathrm{G}$ & $\mathrm{C}$ & 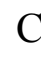 & & & i & $A$ & $\mathrm{C}$ & $\mathrm{T}$ & $\mathrm{G}$ & $\mathrm{T}$ & & & & $\mathrm{C}$ & $\mathrm{A}$ & G & $\mathrm{C}$ & G & 1680 \\
\hline C. aceratus & . & . & . & . & . & . & . & . & . & . & & . & . & . & & & & & & & 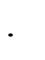 & . & & & & & & & 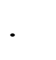 & 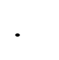 & . & . & 1677 \\
\hline E. maclovinus & . & . & . & . & . & . & . & . & A & . & & $\mathrm{C}$ & . & 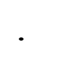 & & • & & & & & & $\cdot$ & & . & & & & & . & & . & & 1677 \\
\hline
\end{tabular}




\begin{tabular}{|c|c|c|c|c|c|c|c|c|c|c|c|c|c|c|c|c|c|c|c|c|c|c|c|c|c|c|c|c|c|c|c|}
\hline$N$. coriiceps & $\mathrm{C}$ & $\mathrm{T}$ & $\mathrm{T}$ & $\mathrm{T}$ & $\mathrm{T}$ & $\mathrm{C}$ & $\mathrm{G}$ & A & $\mathrm{G}$ & $\mathrm{C}$ & $\mathrm{T}$ & $\mathrm{C}$ & A & $\mathrm{A}$ & $\mathrm{C}$ & $\mathrm{T}$ & $\mathrm{T}$ & $\mathrm{C}$ & $\mathrm{T}$ & A & $\mathrm{C}$ & A & G & $\mathrm{C}$ & A & A & $\mathrm{C}$ & G & $\mathrm{G}$ & $\mathrm{C}$ & 1710 \\
\hline C. aceratus & . & . & . & . & . & . & $\cdot$ & . & . & . & • & . & $\cdot$ & . & . & . & . & & & $\cdot$ & $\cdot$ & $\cdot$ & • & $\cdot$ & . & & . & $\cdot$ & & & 1707 \\
\hline E. maclovinus & 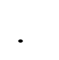 & . & . & . & . & . & $\cdot$ & . & . & . & & $\mathrm{A}$ & . & & 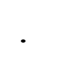 & 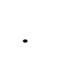 & . & & & & 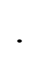 & & . & & . & & $\mathrm{T}$ & & & & 1707 \\
\hline N. coriiceps & $\mathrm{C}$ & $\mathrm{T}$ & $\mathrm{T}$ & $\mathrm{T}$ & $\mathrm{T}$ & $\mathrm{C}$ & $\mathrm{C}$ & A & $\mathrm{T}$ & $\mathrm{G}$ & $\mathrm{T}$ & $\mathrm{C}$ & $\mathrm{T}$ & $\mathrm{T}$ & $\mathrm{C}$ & A & $\mathrm{T}$ & $\mathrm{T}$ & $\mathrm{T}$ & $\mathrm{C}$ & $\mathrm{T}$ & $\mathrm{G}$ & A & $\mathrm{T}$ & $\mathrm{G}$ & $\mathrm{C}$ & A & A & $\mathrm{T}$ & $\mathrm{C}$ & 1740 \\
\hline C. aceratus & . & . & . & . & . & . & . & . & . & . & 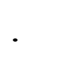 & . & . & . & . & . & . & 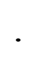 & . & 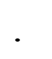 & . & . & . & . & . & 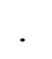 & . & & . & & 1737 \\
\hline E. maclovinus & . & . & . & . & . & . & . & . & . & . & . & . & . & . & . & . & . & $\mathrm{C}$ & • & . & . & . & . & . & . & . & . & . & . & . & 1737 \\
\hline N. coriiceps & $\mathrm{A}$ & $\mathrm{T}$ & $\mathrm{C}$ & $\mathrm{G}$ & $\mathrm{C}$ & $\mathrm{C}$ & $\mathrm{T}$ & $\mathrm{G}$ & $\mathrm{C}$ & $\mathrm{A}$ & $\mathrm{G}$ & $\mathrm{C}$ & $\mathrm{A}$ & $\mathrm{T}$ & $\mathrm{C}$ & $\mathrm{C}$ & $\mathrm{T}$ & G & $\mathrm{T}$ & $\mathrm{C}$ & $\mathrm{C}$ & $\mathrm{C}$ & $\mathrm{T}$ & G & $\mathrm{C}$ & A & $\mathrm{G}$ & $\mathrm{C}$ & $\mathrm{G}$ & $\mathrm{A}$ & 1770 \\
\hline C. aceratus & . & . & . & . & . & . & . & . & . & . & . & . & . & . & . & . & . & . & . & . & . & . & . & . & . & & . & . & . & & 1767 \\
\hline E. maclovinus & . & . & . & . & . & . & . & . & . & . & 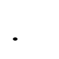 & . & . & . & . & 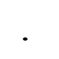 & . & & $\cdot$ & . & A & & . & & 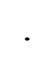 & & . & & . & & 1767 \\
\hline N. coriiceps & $\mathrm{G}$ & A & $\mathrm{G}$ & $\mathrm{C}$ & $\mathrm{T}$ & $\mathrm{G}$ & $\mathrm{G}$ & $\mathrm{T}$ & $\mathrm{G}$ & $\mathrm{G}$ & $\mathrm{C}$ & $\mathrm{T}$ & $\mathrm{G}$ & $\mathrm{A}$ & A & $\mathrm{T}$ & $\mathrm{C}$ & A & $\mathrm{G}$ & A & $\mathrm{G}$ & $\mathrm{T}$ & $\mathrm{C}$ & $\mathrm{C}$ & G & A & $\mathrm{C}$ & $\mathrm{C}$ & A & $\mathrm{C}$ & 1800 \\
\hline C. aceratus & . & . & . & . & . & . & . & . & . & . & . & . & . & . & . & . & . & . & . & . & . & . & . & $\mathrm{T}$ & . & & . & . & $\mathrm{C}$ & & 1797 \\
\hline E. maclovinus & . & . & A & - & . & . & . & . & . & . & . & . & . & . & . & . & . & . & . & . & $\cdot$ & . & . & $\mathrm{T}$ & $\cdot$ & & . & . & . & & 1797 \\
\hline N. coriiceps & $\mathrm{C}$ & $\mathrm{C}$ & $\mathrm{C}$ & $\mathrm{C}$ & $\mathrm{C}$ & $\mathrm{C}$ & G & A & G & $\mathrm{T}$ & G & $\mathrm{T}$ & $\mathrm{C}$ & $\mathrm{T}$ & $\mathrm{C}$ & A & G & $\mathrm{C}$ & A & G & $\mathrm{C}$ & $\mathrm{C}$ & $\mathrm{T}$ & $\mathrm{C}$ & $\mathrm{C}$ & $\mathrm{C}$ & $\mathrm{C}$ & $\mathrm{C}$ & $\mathrm{T}$ & $\mathrm{C}$ & 1830 \\
\hline C. aceratus & . & . & . & . & . & . & . & . & . & G & . & . & . & . & . & 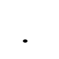 & . & . & ${ }^{\circ}$ & . & . & . & . & 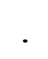 & $\cdot$ & . & . & 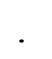 & . & . & 1827 \\
\hline E. maclovinus & . & . & . & . & . & . & . & . & $\mathrm{T}$ & G & & . & . & . & . & . & . & . & . & . & $\cdot$ & . & $\cdot$ & . & . & . & $\mathrm{T}$ & . & $\cdot$ & . & 1827 \\
\hline N. coriiceps & $\mathrm{A}$ & $\mathrm{G}$ & $\mathrm{T}$ & $\mathrm{C}$ & $\mathrm{A}$ & G & G & $\mathrm{A}$ & G & $\mathrm{A}$ & G & $\mathrm{A}$ & $\mathrm{C}$ & $\mathrm{T}$ & $\mathrm{C}$ & A & $\mathrm{T}$ & $\mathrm{C}$ & $\mathrm{C}$ & G & A & A & A & G & $\mathrm{G}$ & $\mathrm{C}$ & $\mathrm{C}$ & G & $\mathrm{C}$ & $\mathrm{C}$ & 1860 \\
\hline C. aceratus & . & . & . & . & . & . & . & . & . & . & 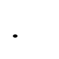 & G & . & . & . & . & . & $\cdot$ & $\cdot$ & $\cdot$ & . & . & . & . & . & . & . & . & $\cdot$ & . & 1857 \\
\hline E. maclovinus & . & . & . & . & . & . & . & . & . & . & . & . & . & . & . & . & . & . & A & & . & . & $\cdot$ & . & . & $\cdot$ & . & . & . & . & 1857 \\
\hline N. coriiceps & G & G & G & $\mathrm{C}$ & $\mathrm{T}$ & $\mathrm{C}$ & $\mathrm{T}$ & $\mathrm{C}$ & $\mathrm{T}$ & $\mathrm{C}$ & $\mathrm{A}$ & $\mathrm{C}$ & $\mathrm{T}$ & $\mathrm{T}$ & $\mathrm{C}$ & $\mathrm{C}$ & $\mathrm{T}$ & $\mathrm{T}$ & G & $\mathrm{T}$ & $\mathrm{C}$ & A & A & $\mathrm{T}$ & G & A & A & $\mathrm{G}$ & $\mathrm{T}$ & G & 1890 \\
\hline C. aceratus & . & . & . & . & . & . & . & . & . & . & . & . & . & . & . & . & . & 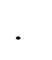 & . & . & . & . & . & . & 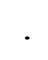 & . & . & . & . & 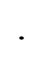 & 1887 \\
\hline E. maclovinus & . & . & . & . & . & . & & . & A & . & & 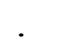 & . & 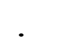 & . & • & • & & & & $\cdot$ & & . & & 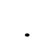 & & • & & . & & 1887 \\
\hline
\end{tabular}




\begin{tabular}{|c|c|c|c|c|c|c|c|c|c|c|c|c|c|c|c|c|c|c|c|c|c|c|c|c|c|c|c|c|c|c|c|c|c|}
\hline N. coriiceps & $\mathrm{A}$ & $\mathrm{C}$ & A & $\mathrm{C}$ & $\mathrm{T}$ & $\mathrm{G}$ & $\mathrm{G}$ & $\mathrm{C}$ & A & $\mathrm{C}$ & $\mathrm{C}$ & $\mathrm{A}$ & $\mathrm{C}$ & $\mathrm{C}$ & $\mathrm{A}$ & 1 & C & & & $\mathrm{C}$ & $\mathrm{A}$ & $\mathrm{G}$ & A & & 1 & & & & & & & $\mathrm{C}$ & 1920 \\
\hline C. aceratus & . & . & . & . & • & . & $\cdot$ & . & . & . & . & . & $\cdot$ & . & $\mathrm{C}$ & & $\cdot$ & & & & & . & & & & & & & & & & & 1917 \\
\hline E. maclovinus & . & . & $\mathrm{G}$ & 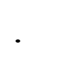 & . & . & & 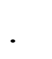 & 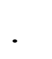 & . & & . & 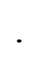 & . & $\mathrm{C}$ & & & & & & & & & & & & & & & & & & 1917 \\
\hline N. coriiceps & $\mathrm{C}$ & A & $\mathrm{G}$ & $\mathrm{G}$ & $\mathrm{T}$ & $\mathrm{T}$ & $\mathrm{T}$ & $\mathrm{T}$ & $\mathrm{C}$ & $\mathrm{C}$ & A & $\mathrm{T}$ & $\mathrm{G}$ & A & $\mathrm{C}$ & G & ( & & & $G$ & $\mathrm{~T}$ & A & $\mathrm{G}$ & $\mathrm{C}$ & G & ( & & & & & T & $\mathrm{C}$ & 1950 \\
\hline C. aceratus & . & . & . & . & . & . & . & . & . & . & . & $\cdot$ & . & . & $\cdot$ & & & & & $A$ & 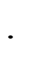 & . & 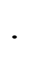 & & A & & & & & & & & 1947 \\
\hline E. maclovinus & . & . & . & . & $\cdot$ & . & . & . & $\cdot$ & . & • & . & $\cdot$ & . & $\cdot$ & & & & & & . & $\mathrm{C}$ & A & & 0 & & & & & & & & 1947 \\
\hline N. coriiceps & A & $\mathrm{T}$ & $\mathrm{T}$ & $\mathrm{C}$ & $\mathrm{A}$ & $\mathrm{A}$ & $\mathrm{T}$ & $\mathrm{A}$ & $\mathrm{T}$ & G & $\mathrm{G}$ & A & $\mathrm{G}$ & $\mathrm{T}$ & $\mathrm{T}$ & $\mathrm{C}$ & 1 & & $y$ & T & $\mathrm{A}$ & $\mathrm{C}$ & G & $\mathrm{T}$ & $\mathrm{A}$ & ( & & & & $\mathrm{J}$ & $A$ & G & 1980 \\
\hline C. aceratus & . & . & $\mathrm{C}$ & . & . & . & . & . & . & . & . & $\cdot$ & . & $\cdot$ & . & & . & & & & . & . & 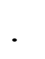 & & . & • & & & & & & & 1977 \\
\hline E. maclovinus & . & . & $\mathrm{C}$ & - & . & G & 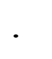 & . & . & . & . & 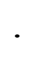 & . & . & . & & & & & & . & . & & & . & & & & & & & & 1977 \\
\hline N. coriiceps & G & A & $\mathrm{G}$ & G & A & $\mathrm{C}$ & $\mathrm{C}$ & A & G & G & A & G & G & A & A & $\mathrm{C}$ & 7 & & i & $A$ & $\mathrm{G}$ & $\mathrm{T}$ & $\mathrm{C}$ & $\mathrm{C}$ & C & & & & & C & $\mathrm{C}$ & $\mathrm{C}$ & 2010 \\
\hline C. aceratus & . & . & . & . & . & . & . & . & . & . & $\cdot$ & $\cdot$ & . & . & $\cdot$ & $\cdot$ & 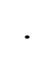 & & & . & 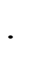 & 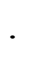 & . & & . & . & & & & & & . & 2007 \\
\hline E. maclovinus & . & . & . & . & . & . & . & . & $\cdot$ & . & . & $\cdot$ & . & . & $\cdot$ & & • & & & . & 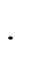 & $\cdot$ & . & & . & & & & & & & & 2007 \\
\hline N. coriiceps & $\mathrm{A}$ & $\mathrm{C}$ & $\mathrm{A}$ & $\mathrm{G}$ & $\mathrm{A}$ & $\mathrm{C}$ & $\mathrm{G}$ & $\mathrm{A}$ & A & $\mathrm{C}$ & $\mathrm{C}$ & $\mathrm{T}$ & $\mathrm{T}$ & G & G & $\mathrm{C}$ & ( & & & A & $\mathrm{A}$ & A & A & A & $\mathrm{G}$ & 1 & & & & & $\mathrm{C}$ & A & 2040 \\
\hline C. aceratus & . & . & . & . & . & $\mathrm{G}$ & . & . & . & . & - & . & . & . & . & . & & & & . & . & . & & & . & • & & & & & & . & 2037 \\
\hline E. maclovinus & . & . & . & . & . & G & . & . & . & . & $\cdot$ & A & · & $\cdot$ & 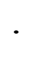 & . & 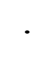 & & & · & & . & & & . & • & & & & & & & 2037 \\
\hline N. coriiceps & G & A & G & $\mathrm{C}$ & $\mathrm{C}$ & $\mathrm{C}$ & $\mathrm{C}$ & $\mathrm{T}$ & $\mathrm{T}$ & $\mathrm{T}$ & $\mathrm{C}$ & $\mathrm{C}$ & $\mathrm{T}$ & G & G & A & ( & & a & A & $\mathrm{G}$ & $\mathrm{T}$ & $\mathrm{G}$ & A & $\mathrm{C}$ & & & & i & J & A & $\mathrm{G}$ & 2070 \\
\hline C. aceratus & . & . & . & . & . & . & $\cdot$ & . & $\cdot$ & . & . & $\cdot$ & . & . & • & & $\cdot$ & & & . & . & . & & . & . & . & & & & & & . & 2067 \\
\hline E. maclovinus & . & . & . & . & . & . & . & . & . & . & & . & . & . & & $\cdot$ & . & & & . & . & $\mathrm{C}$ & & . & . & & & & & & & . & 2067 \\
\hline N. coriiceps & G & A & $\mathrm{T}$ & $\mathrm{G}$ & A & $\mathrm{G}$ & G & A & $\mathrm{C}$ & A & $\mathrm{G}$ & $\mathrm{T}$ & G & A & $\mathrm{C}$ & $\mathrm{T}$ & 1 & & 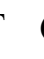 & G & $\mathrm{G}$ & A & $\mathrm{G}$ & A & G & & & & & $y$ & A & G & 2100 \\
\hline C. aceratus & . & . & . & . & . & . & . & . & . & . & & . & . & . & & & & & & . & & . & & & & . & & & & & & . & 2097 \\
\hline E. maclovinus & . & . & . & . & . & . & . & . & . & . & & . & . & . & & & & & & & & . & & . & & . & & & & & & & 2097 \\
\hline
\end{tabular}




\begin{tabular}{|c|c|c|c|c|c|c|c|c|c|c|c|c|c|c|c|c|c|c|c|c|c|c|c|c|c|c|c|c|c|c|c|c|c|}
\hline N. coriiceps & A & $\mathrm{G}$ & $\mathrm{A}$ & $\mathrm{G}$ & $\mathrm{A}$ & $\mathrm{T}$ & $\mathrm{C}$ & G & $\mathrm{C}$ & $\mathrm{T}$ & A & $\mathrm{T}$ & $\mathrm{C}$ & $\mathrm{T}$ & & A & & & $\mathrm{G}$ & G & $\mathrm{T}$ & $\mathrm{G}$ & $\mathrm{A}$ & C & & & & $\mathrm{T}$ & $\mathrm{T}$ & $\mathrm{T}$ & $\mathrm{C}$ & $\mathrm{C}$ & 2130 \\
\hline C. aceratus & . & . & $\cdot$ & $\cdot$ & . & . & $\cdot$ & . & . & . & . & . & $\cdot$ & . & & . & & & & $\cdot$ & & . & & & & & & & $\cdot$ & & . & & 2127 \\
\hline E. maclovinus & & . & . & . & . & . & & 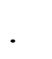 & 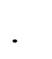 & 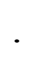 & & 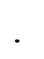 & 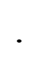 & & & & & & & & & & & & & & & & & & . & & 2127 \\
\hline N. coriiceps & G & $\mathrm{C}$ & $\mathrm{A}$ & $\mathrm{G}$ & $\mathrm{A}$ & $\mathrm{G}$ & $\mathrm{C}$ & A & $\mathrm{T}$ & $\mathrm{C}$ & A & G & $\mathrm{G}$ & A & $\mathrm{G}$ & 1 & & 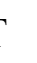 & $\mathrm{C}$ & $\mathrm{T}$ & $\mathrm{T}$ & $\mathrm{C}$ & G & $\mathrm{T}$ & & & $\Gamma$ & $\mathrm{T}$ & $\mathrm{C}$ & $\mathrm{C}$ & $\mathrm{T}$ & A & 2160 \\
\hline C. aceratus & . & . & . & . & . & . & . & . & . & . & . & $\cdot$ & . & . & & & . & & & $\cdot$ & . & . & & & & & & & 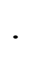 & & 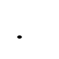 & G & 2157 \\
\hline E. maclovinus & . & . & . & . & . & . & . & . & . & . & • & • & $\cdot$ & . & & 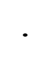 & . & & & $\cdot$ & & . & & & & & & & . & • & . & . & 2157 \\
\hline N. coriiceps & $\mathrm{C}$ & $\mathrm{A}$ & $\mathrm{G}$ & $\mathrm{C}$ & $\mathrm{G}$ & $\mathrm{A}$ & $\mathrm{C}$ & $\mathrm{T}$ & $\mathrm{C}$ & $\mathrm{G}$ & $\mathrm{T}$ & $\mathrm{C}$ & A & G & $\mathrm{C}$ & C & & $y$ & $\mathrm{C}$ & G & $\mathrm{T}$ & $\mathrm{G}$ & $\mathrm{C}$ & $\mathrm{T}$ & & J & $\mathrm{G}$ & A & $\mathrm{G}$ & $\mathrm{G}$ & $\mathrm{C}$ & $\mathrm{C}$ & 2190 \\
\hline C. aceratus & $\cdot$ & . & . & . & . & . & . & . & . & . & $\cdot$ & 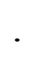 & . & $\cdot$ & • & . & . & & - & $\cdot$ & . & ${ }^{\circ}$ & . & . & & & & & . & . & . & . & 2187 \\
\hline E. maclovinus & . & . & . & . & . & - & . & . & $\mathrm{T}$ & . & . & . & . & . & • & . & . & & 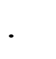 & . & $\cdot$ & . & . & • & & & & & . & . & . & . & 2187 \\
\hline N. coriiceps & $\mathrm{T}$ & A & $\mathrm{C}$ & A & G & $\mathrm{C}$ & G & G & $\mathrm{C}$ & G & $\mathrm{C}$ & $\mathrm{C}$ & G & $\mathrm{C}$ & $\mathrm{C}$ & $A$ & & 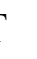 & $\mathrm{C}$ & $\mathrm{T}$ & $\mathrm{T}$ & $\mathrm{T}$ & G & $\mathrm{T}$ & & 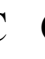 & C & A & $\mathrm{C}$ & A & G & $\mathrm{T}$ & 2220 \\
\hline C. aceratus & $\cdot$ & . & . & . & . & . & . & . & . & . & . & $\cdot$ & . & $\cdot$ & • & . & . & & 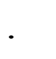 & $\cdot$ & 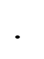 & . & . & 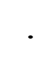 & & & & . & . & . & . & . & 2217 \\
\hline E. maclovinus & . & . & . & . & . & . & . & . & . & . & . & . & . & . & . & . & . & & . & . & & $\cdot$ & . & $\cdot$ & & & & . & . & . & . & . & 2217 \\
\hline N. coriiceps & $\mathrm{C}$ & $\mathrm{T}$ & G & A & $\mathrm{G}$ & $\mathrm{T}$ & $\mathrm{C}$ & $\mathrm{A}$ & $\mathrm{G}$ & $\mathrm{C}$ & $\mathrm{C}$ & $\mathrm{C}$ & A & $\mathrm{T}$ & $\mathrm{G}$ & C & & 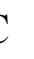 & $\mathrm{C}$ & G & A & $\mathrm{G}$ & $\mathrm{T}$ & C & & 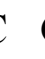 & $\mathrm{G}$ & A & $\mathrm{C}$ & $\mathrm{T}$ & $\mathrm{A}$ & $\mathrm{C}$ & 2250 \\
\hline C. aceratus & . & . & . & . & . & . & . & . & . & . & . & . & $\cdot$ & . & . & • & & & - & $\cdot$ & $\cdot$ & . & . & & & & & & . & . & . & . & 2247 \\
\hline E. maclovinus & . & . & . & . & . & $\mathrm{C}$ & . & . & . & . & . & $\cdot$ & . & $\cdot$ & . & 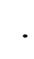 & & & - & $\cdot$ & $\cdot$ & $\cdot$ & . & • & & & & & . & . & . & . & 2247 \\
\hline N. coriiceps & A & $\mathrm{C}$ & $\mathrm{C}$ & $\mathrm{C}$ & A & $\mathrm{G}$ & $\mathrm{C}$ & G & $\mathrm{G}$ & $\mathrm{C}$ & $\mathrm{T}$ & $\mathrm{C}$ & $\mathrm{T}$ & $\mathrm{T}$ & $\mathrm{C}$ & $A$ & & $\mathrm{~J}$ & A & $\mathrm{T}$ & A & $\mathrm{C}$ & $\mathrm{C}$ & $\mathrm{T}$ & & I & C & $\mathrm{T}$ & $\mathrm{C}$ & A & $\mathrm{C}$ & A & 2280 \\
\hline C. aceratus & $\cdot$ & . & . & . & . & . & $\cdot$ & . & . & . & . & $\cdot$ & . & $\cdot$ & . & • & . & & . & . & . & . & . & • & 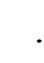 & . & & . & . & . & . & . & 2277 \\
\hline E. maclovinus & . & . & . & . & 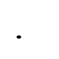 & . & A & . & . & . & & . & . & . & . & 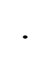 & & & & $\cdot$ & $\cdot$ & . & $\cdot$ & & & & & & . & . & . & . & 2277 \\
\hline N. coriiceps & $\mathrm{C}$ & $\mathrm{G}$ & $\mathrm{C}$ & A & $\mathrm{C}$ & $\mathrm{A}$ & G & A & $\mathrm{G}$ & A & $\mathrm{G}$ & A & G & $\mathrm{G}$ & A & C & & 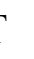 & G & G & $\mathrm{C}$ & $\mathrm{T}$ & G & $C$ & & 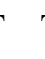 & $\Gamma$ & A & $\mathrm{T}$ & G & $\mathrm{G}$ & $\mathrm{T}$ & 2310 \\
\hline C. aceratus & . & . & . & . & . & . & . & . & . & . & & 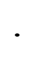 & 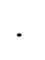 & . & & & & & . & . & & . & 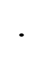 & & & & & · & . & " & . & 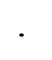 & 2307 \\
\hline E. maclovinus & $\cdot$ & . & . & . & . & $\mathrm{C}$ & $\cdot$ & . & . & . & & . & . & . & • & & & & . & $\cdot$ & & . & & & & & & & . & & . & & 2307 \\
\hline
\end{tabular}


N. coriiceps

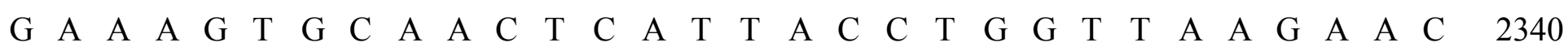

C. aceratus

E. maclovinus

N. coriiceps

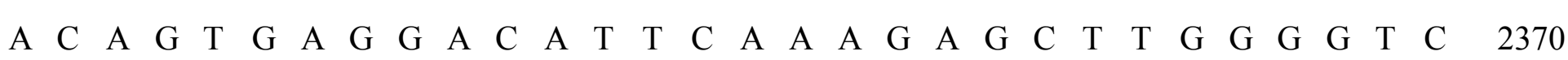

C. aceratus

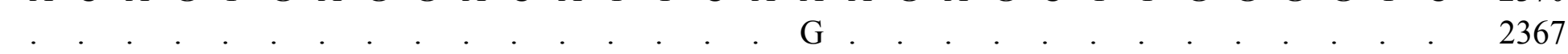

E. maclovinus

N. coriiceps

C. aceratus

C $\mathrm{T}$ T A A $\mathrm{G}$

C A G

A G A A

A A G A G A A

C C

A $G \mathrm{G}$

2367

E. maclovinus

N. coriiceps

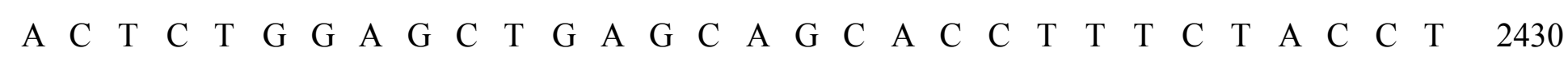

C. aceratus

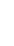

E. maclovinus

N. coriiceps

C A G G .

2427

C. aceratus

E. maclovinus

N. coriiceps

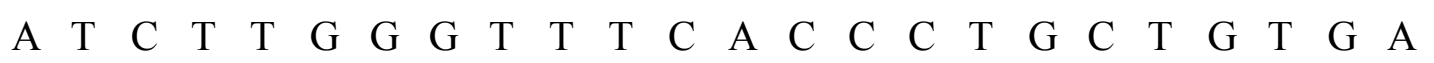

2484

C. aceratus

2481

E. maclovinus

2481 


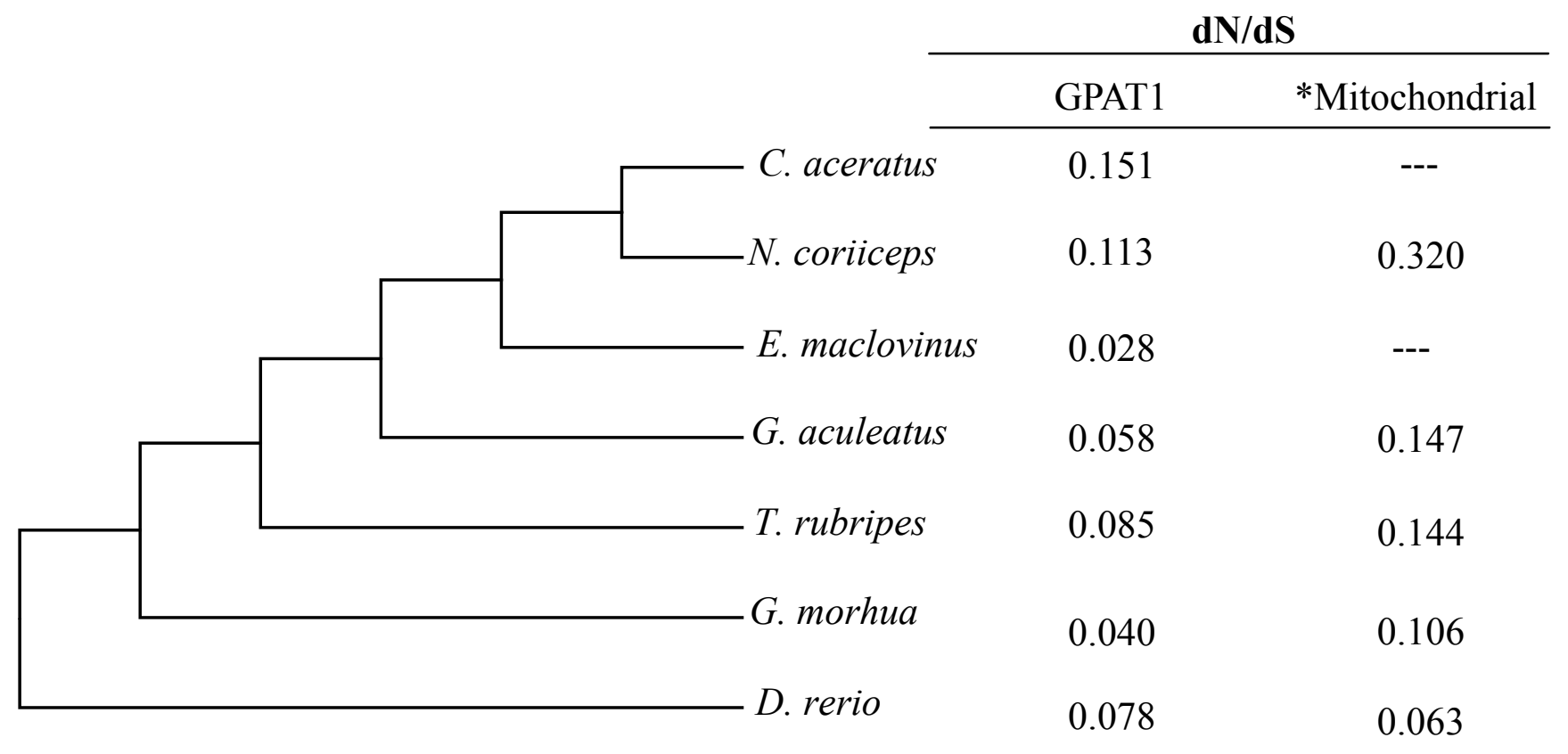


N. coriiceps

C. aceratus

E. maclovinus

G. aculeatus

T. rubripes

M. zebra

P. nyererei

$O$. niloticus

H. sapiens

M. musculus

$R$. norvegicus

B. taurus

M. gallopavo

G. gallus

N. coriiceps

C. aceratus

E. maclovinus

G. aculeatus

T. rubripes

M. zebra

P. nyererei

$O$. niloticus

H. sapiens

M. musculus

$R$. norvegicus

B. taurus

M. gallopavo

G. gallus

N. coriiceps

C. aceratus

E. maclovinus

G. aculeatus

T. rubripes

M. zebra

$P$. nyererei

O. niloticus

H. sapiens

M. musculus

R. norvegicus

B. taurus

M. gallopavo

G. gallus

N. coriiceps

C. aceratus

E. maclovinus

G. aculeatus

T. rubripes

M. zebra

P. nyererei

O. niloticus

H. sapiens

M. musculus

R. norvegicus

B. taurus

M. gallopavo

G. gallus

N. coriiceps

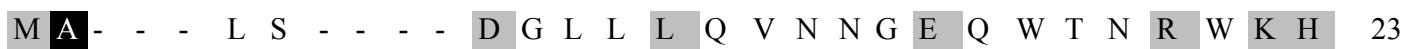

M A - - - $\mathrm{L}$ S - - - - D G L L L Q V N N G E Q W T N R W

M E - - - $\mathrm{L}$ S - - - - $\mathrm{D}$ G L L L Q V N N G E Q W T N R W

M D - - - $\mathrm{L}$ S - - - - $\mathrm{D}$ G L L L Q V N N G E Q W C N R W

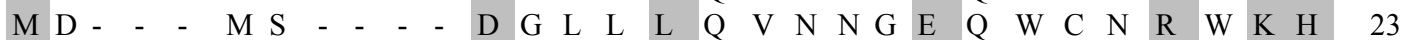

M E - - - $\mathrm{L}$ S - - - - D G L L L Q V S N G E Q W C N R W

M E - - - L S - - - - D G L L L Q V S N G E Q W C N R W

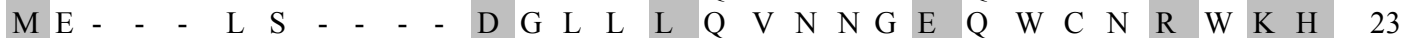

$\begin{array}{lllllllllllllllllllllllllllllll}\text { M D E } & \text { S } & \text { A } & \text { L } & \text { T } & \text { L } & G & \text { T } & \text { I } & \text { D } & \text { V } & \text { S } & \text { Y } & \text { L } & \text { P } & \text { H } & \text { S } & \text { S } & - & \text { E } & \text { Y } & \text { S } & \text { V } & \text { G } & \text { R } & \text { C } & \text { K } & \text { H } & 29\end{array}$

$\begin{array}{llllllllllllllllllllllllllllllll}\text { M E } & \text { E } & \text { S } & \text { S } & \text { V } & \text { T } & \text { V } & G & \text { T } & \text { I } & \text { D } & \text { V } & \text { S } & \text { Y } & \text { L } & \text { P } & \text { S } & \text { S } & \text { S } & - & \text { E } & \text { Y } & \text { S } & \text { L } & \text { G } & \text { R } & \text { C } & \text { K } & \text { H } & 29\end{array}$

$\begin{array}{lllllllllllllllllllllllllllllll}M & E & E & S & S & V & T & I & G & T & I & D & V & S & Y & L & P & N & S & S & - & E & Y & S & L & G & R & C & K & H & 29\end{array}$

$\begin{array}{lllllllllllllllllllllllllllllll}\text { M D D E } & \text { S } & \text { A } & \text { L } & \text { T } & \text { L } & G & \text { T } & \text { I } & \text { D } & \text { V } & \text { S } & \text { Y } & \text { L } & \text { P } & \text { N } & \text { S } & \text { S } & - & \text { E } & \text { Y } & \text { S } & \text { I } & \text { G } & \text { R } & \text { C } & \text { K } & \text { H } & 29\end{array}$

$\begin{array}{lllllllllllllllllllllllllllllll}\text { M D E } & \text { T } & \text { A } & \text { L } & \text { S } & \text { L } & G & \text { T } & \text { I } & \text { D } & \text { V } & \text { S } & \text { Y } & \text { L } & \text { S } & \text { T } & \text { S } & \text { A } & - & \text { E } & \text { C } & \text { S } & \text { V } & \text { S } & \text { R } & \text { C } & \text { K } & \text { H } & 29\end{array}$

$\begin{array}{llllllllllllllllllllllllllllll}\text { M D E } & \text { T } & \text { A } & \text { L } & \text { S } & \text { L } & G & \text { T } & \text { I } & \text { D } & \text { V } & \text { S } & \text { Y } & \text { L } & \text { S } & \text { T } & \text { S } & \text { A } & - & \text { E } & \text { C } & \text { S } & \text { V } & \text { S } & \text { R } & \text { C } & \text { K } & \text { H } & 29\end{array}$

$\begin{array}{lllllllllllllllllllllllllllllll}\text { P } & \text { N D D } & \text { D S S } & - & \text { D } & \text { R S S } & \text { T } & \text { S } & \text { P } & \text { S } & \text { V } & \text { L } & \text { R } & \text { C } & \text { V } & \text { A } & \text { S } & \text { T } & \text { W } & \text { K } & \text { E } & \text { G } & \text { L } & \text { L } & \text { S } & \text { R } & \text { K } & 52\end{array}$ $\begin{array}{llllllllllllllllllllllllllllll}\text { P } & \text { N D D } & \text { D } & \text { S } & - & \text { D } & \text { R } & \text { S } & \text { T } & \text { S } & \text { P } & \text { S } & \text { V } & \text { L } & \text { R } & \text { C } & \text { V } & \text { A } & \text { S } & \text { T } & \text { W } & \text { K } & \text { E } & \text { G } & \text { L } & \text { L } & \text { S } & \text { R } & \text { K } & 52\end{array}$

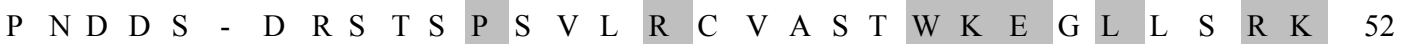

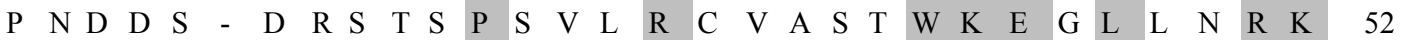
$\begin{array}{llllllllllllllllllllllllll}\text { P N D D L } & \text { - } & \text { D } & \text { R S } & \text { T } & \text { S } & \text { P } & \text { S } & \text { V } & \text { L } & \text { R } & \text { C } & \text { V } & \text { A } & \text { S } & \text { T } & \text { W } & \text { K } & \text { E } & \text { G } & \text { L } & \text { L } & \text { N } & \text { R } & \text { K } & 52\end{array}$

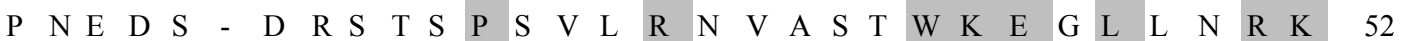
$\begin{array}{lllllllllllllllllllllllllllllll}\text { P } & N & E & D & S & - & D & R & S & T & S & P & S & V & L & R & N & V & A & S & T & \text { W } & \text { K } & \text { E } & \text { G } & \text { L } & \text { L } & \text { N } & \text { R } & \text { K } & 52\end{array}$ $\begin{array}{lllllllllllllllllllllllllllllll}\mathrm{P} & \mathrm{N} & \mathrm{E} & \mathrm{E} & \mathrm{S} & - & \mathrm{D} & \mathrm{R} & \mathrm{S} & \mathrm{T} & \mathrm{S} & \mathrm{P} & \mathrm{S} & \mathrm{V} & \mathrm{L} & \mathrm{R} & \mathrm{N} & \mathrm{V} & \mathrm{A} & \mathrm{S} & \mathrm{T} & \mathrm{W} & \mathrm{K} & \mathrm{E} & \mathrm{G} & \mathrm{L} & \mathrm{L} & \mathrm{N} & \mathrm{R} & \mathrm{K} & 52\end{array}$ $\begin{array}{llllllllllllllllllllllllllllllll}T & S & E & E & W & G & E & C & G & F & R & P & T & I & F & R & S & A & T & L & K & W & K & E & S & L & M & S & R & K & 59\end{array}$ $\begin{array}{lllllllllllllllllllllllllllllllll}\text { T } & \text { S } & \text { E } & D & \text { W } & \text { V } & \text { D } & \text { C } & G & F & K & P & \text { T } & \text { F } & \text { F } & \text { R } & \text { S } & \text { A } & \text { T } & \text { L } & \text { K } & \text { W } & \text { K } & \text { E } & \text { S } & \text { L } & \text { M } & \text { S } & \text { R } & \text { K } & 59\end{array}$

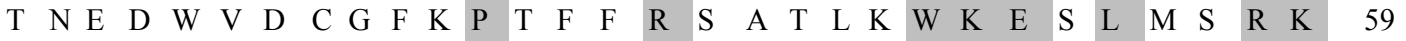
$\begin{array}{lllllllllllllllllllllllllllllllll}\text { A } & T & E & E & W & G & E & C & G & S & R & P & T & V & F & R & S & A & T & L & K & W & K & E & S & L & M & S & R & K & 59\end{array}$

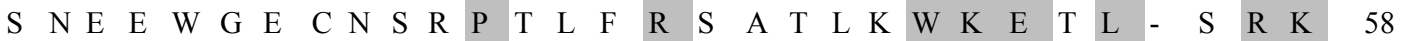

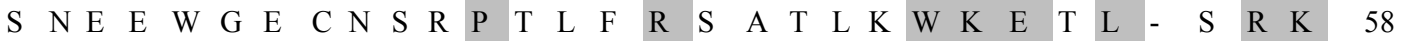

$\begin{array}{lllllllllllllllllllllllllllllllll}R & P & F & V & G & R & C & C & H & S & C & T & P & Q & S & \text { W } & E & K & L & F & N & P & S & I & P & S & L & G & L & R & 82\end{array}$ $\begin{array}{llllllllllllllllllllllllllllllll}R & P & F & V & G & R & C & C & H & S & C & T & P & Q & S & \text { W } & E & K & L & F & N & P & S & I & P & S & L & G & L & R & 82\end{array}$ $\begin{array}{llllllllllllllllllllllllllllllll}R & P & F & V & G & R & C & C & H & S & C & T & P & Q & S & \text { W } & E & K & L & F & N & P & S & I & P & S & L & G & L & R & 82\end{array}$ $\begin{array}{llllllllllllllllllllllllllllllllll}R & P & F & V & G & R & C & C & H & S & C & T & P & Q & S & \text { W } & E & K & L & F & N & P & S & I & P & S & L & G & L & R & 82\end{array}$ $\begin{array}{llllllllllllllllllllllllllllllll}R & P & F & V & G & R & C & C & H & S & C & T & P & Q & S & \text { W } & E & K & L & F & N & P & S & I & P & S & L & G & L & R & 82\end{array}$ $\begin{array}{llllllllllllllllllllllllllllllll}\mathrm{R} & \mathrm{P} & \mathrm{F} & \mathrm{V} & \mathrm{G} & \mathrm{R} & \mathrm{C} & \mathrm{C} & \mathrm{H} & \mathrm{S} & \mathrm{C} & \mathrm{T} & \mathrm{P} & \mathrm{Q} & \mathrm{S} & \mathrm{W} & \mathrm{E} & \mathrm{R} & \mathrm{L} & \mathrm{F} & \mathrm{N} & \mathrm{P} & \mathrm{S} & \mathrm{I} & \mathrm{P} & \mathrm{S} & \mathrm{L} & \mathrm{G} & \mathrm{L} & \mathrm{R} & 82\end{array}$ $\begin{array}{llllllllllllllllllllllllllllllllll}R & P & F & V & G & R & C & C & H & S & C & T & P & Q & S & W & E & R & L & F & N & P & S & I & P & S & L & G & L & R & 82\end{array}$ $\begin{array}{llllllllllllllllllllllllllllllll}R & P & F & V & G & R & C & C & H & S & C & T & P & Q & S & \text { W } & E & R & L & F & N & P & S & I & P & S & L & G & L & R & 82\end{array}$ $\begin{array}{lllllllllllllllllllllllllllllllll}R & P & F & V & G & R & C & C & Y & S & C & T & P & Q & S & \text { W } & D & K & F & F & N & P & S & I & P & S & L & G & L & R & 89\end{array}$ $\begin{array}{lllllllllllllllllllllllllllllllll}R & P & F & V & G & R & C & C & Y & S & C & T & P & Q & S & \text { W } & E & R & F & F & N & P & S & I & P & S & L & G & L & R & 89\end{array}$ $\begin{array}{llllllllllllllllllllllllllllllll}R & P & F & V & G & R & C & C & Y & S & C & T & P & Q & S & \text { W } & E & R & F & F & N & P & S & I & P & S & L & G & L & R & 89\end{array}$ $\begin{array}{lllllllllllllllllllllllllllllllll}R & P & F & V & G & R & C & C & Y & S & C & T & P & Q & S & \text { W } & D & K & F & F & N & P & S & I & P & S & L & G & L & R & 89\end{array}$ $\begin{array}{llllllllllllllllllllllllllllllllll}R & P & F & V & G & R & C & C & Y & V & C & T & P & Q & S & R & D & N & F & F & N & A & S & I & P & S & L & G & L & R & 88\end{array}$

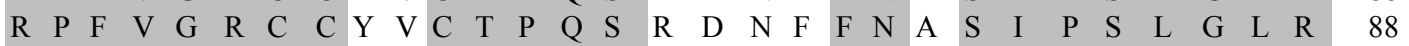

$\begin{array}{llllllllllllllllllllllllllllll}\text { N } & \text { V I I } & \text { F } & \text { I } & \text { N } & \text { E } & \text { T } & \text { H } & \text { T } & - & - & \text { R } & \text { Q } & \text { R } & \text { G } & \text { W } & \text { L } & \text { A } & R & R & \text { L } & \text { S } & \text { Y } & \text { V } & \text { L } & \text { F } & \text { V } & \text { M } & \text { E } & 110\end{array}$

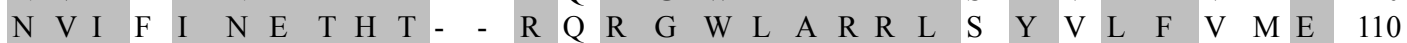
$\begin{array}{llllllllllllllllllllllllllllllll}\mathrm{N} & \mathrm{V} & \mathrm{I} & \mathrm{F} & \mathrm{I} & \mathrm{N} & \mathrm{E} & \mathrm{T} & \mathrm{H} & \mathrm{T} & - & - & \mathrm{R} & \mathrm{Q} & \mathrm{R} & \mathrm{G} & \mathrm{W} & \mathrm{L} & \mathrm{A} & \mathrm{R} & \mathrm{R} & \mathrm{L} & \mathrm{S} & \mathrm{Y} & \mathrm{V} & \mathrm{L} & \mathrm{F} & \mathrm{V} & \mathrm{M} & \mathrm{E} & 110\end{array}$ $\begin{array}{lllllllllllllllllllllllllllllll}\text { N } & \text { V } & \text { I } & \text { Y } & \text { I } & \text { N } & \text { E } & \text { T } & \text { H } & \text { T } & \text { R } & \text { C } & R & \text { Q } & R & \text { G } & \text { W } & \text { L } & \text { A } & R & R & \text { L } & \text { S } & \text { Y } & \text { V } & \text { L } & \text { F } & \text { V } & \text { M } & \text { E } & 112\end{array}$

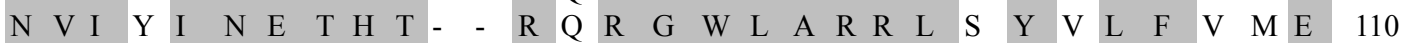
$\begin{array}{lllllllllllllllllllllllllllllll}N & \text { V I } & \text { Y } & \text { I } & \text { N } & \text { E } & \text { T } & H & \text { T } & - & - & R & Q & R & G & \text { W } & \text { L } & \text { A } & R & R & \text { L } & \text { S } & \text { Y } & \text { V } & \text { L } & \text { F } & \text { V } & \text { V } & \text { E } & 110\end{array}$

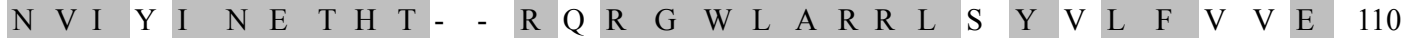

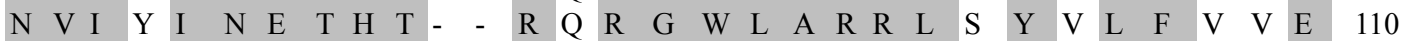
$\begin{array}{llllllllllllllllllllllllllllllll}\mathrm{N} & \mathrm{V} & \mathrm{I} & \mathrm{Y} & \mathrm{I} & \mathrm{N} & \mathrm{E} & \mathrm{T} & \mathrm{H} & \mathrm{T} & - & - & \mathrm{R} & \mathrm{H} & \mathrm{R} & \mathrm{G} & \mathrm{W} & \mathrm{L} & \mathrm{A} & \mathrm{R} & \mathrm{R} & \mathrm{L} & \mathrm{S} & \mathrm{Y} & \mathrm{V} & \mathrm{L} & \mathrm{F} & \mathrm{I} & \mathrm{Q} & \mathrm{E} & 117\end{array}$

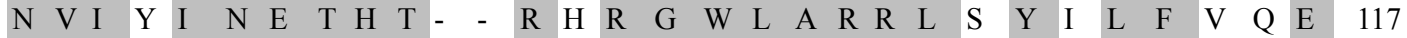

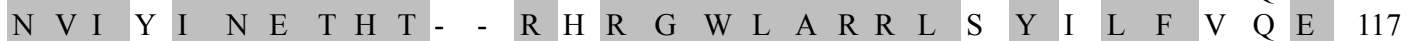

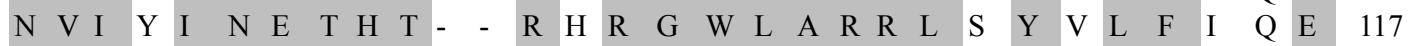
$\begin{array}{llllllllllllllllllllllllllllllll}\mathrm{N} & \mathrm{V} & \mathrm{I} & \mathrm{Y} & \mathrm{I} & \mathrm{N} & \mathrm{E} & \mathrm{T} & \mathrm{H} & \mathrm{T} & - & - & \mathrm{R} & \mathrm{Y} & \mathrm{R} & \mathrm{G} & \mathrm{W} & \mathrm{L} & \mathrm{A} & \mathrm{R} & \mathrm{R} & \mathrm{L} & \mathrm{C} & \mathrm{Y} & \mathrm{V} & \mathrm{L} & \mathrm{F} & \mathrm{V} & \mathrm{L} & \mathrm{E} & 116\end{array}$

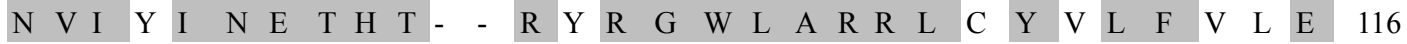


C. aceratus

E. maclovinus

G. aculeatus

T. rubripes

M. zebra

P. nyererei

$O$. niloticus

$H$. sapiens

M. musculus

$R$. norvegicus

B. taurus

M. gallopavo

G. gallus

N. coriiceps

C. aceratus

E. maclovinus

G. aculeatus

T. rubripes

M. zebra

P. nyererei

O. niloticus

H. sapiens

M. musculus

$R$. norvegicus

B. taurus

M. gallopavo

G. gallus

$N$. coriiceps

C. aceratus

E. maclovinus

G. aculeatus

T. rubripes

M. zebra

P. nyererei

O. niloticus

$H$. sapiens

M. musculus

$R$. norvegicus

B. taurus

M. gallopavo

G. gallus

N. coriiceps

C. aceratus

E. maclovinus

G. aculeatus

T. rubripes

M. zebra

$P$. nyererei

$O$. niloticus

H. sapiens

M. musculus

$R$. norvegicus

B. taurus

M. gallopavo

G. gallus

N. coriiceps

C. aceratus
$\begin{array}{lllllllllllllllllllllllllllllllllll}R & D & V & N & K & D & M & F & T & R & N & V & V & D & N & V & L & N & N & S & R & V & E & S & A & I & A & D & V & A & 140\end{array}$ $\begin{array}{lllllllllllllllllllllllllllllllll}R & D & V & N & K & D & M & F & T & R & N & V & V & D & N & V & L & N & N & C & R & V & E & S & A & I & A & N & V & A & 140\end{array}$ $\begin{array}{llllllllllllllllllllllllllllllll}R & D & V & N & K & D & M & F & T & R & N & V & V & D & N & V & L & N & S & N & G & V & E & C & S & \text { I } & \text { A } & \text { E } & \text { V } & \text { A } & 142\end{array}$ $\begin{array}{lllllllllllllllllllllllllllllllll}R & D & V & N & K & D & M & F & T & R & N & I & V & D & N & V & L & N & N & K & K & V & E & N & A & I & E & K & V & A & 140\end{array}$ $\begin{array}{lllllllllllllllllllllllllllllllll}\mathrm{R} & \mathrm{D} & \mathrm{V} & \mathrm{H} & \mathrm{K} & \mathrm{D} & \mathrm{M} & \mathrm{F} & \mathrm{T} & \mathrm{R} & \mathrm{N} & \mathrm{V} & \mathrm{V} & \mathrm{D} & \mathrm{N} & \mathrm{V} & \mathrm{L} & \mathrm{N} & \mathrm{N} & \mathrm{S} & \mathrm{R} & \mathrm{V} & \mathrm{E} & \mathrm{D} & \mathrm{A} & \mathrm{I} & \mathrm{V} & \mathrm{T} & \mathrm{V} & \mathrm{A} & 140\end{array}$ $\begin{array}{lllllllllllllllllllllllllllllllll}\mathrm{R} & \mathrm{D} & \mathrm{V} & \mathrm{H} & \mathrm{K} & \mathrm{D} & \mathrm{M} & \mathrm{F} & \mathrm{T} & \mathrm{R} & \mathrm{N} & \mathrm{M} & \mathrm{V} & \mathrm{D} & \mathrm{N} & \mathrm{V} & \mathrm{L} & \mathrm{N} & \mathrm{N} & \mathrm{S} & \mathrm{R} & \mathrm{V} & \mathrm{E} & \mathrm{D} & \mathrm{A} & \mathrm{I} & \mathrm{V} & \mathrm{T} & \mathrm{V} & \mathrm{A} & 140\end{array}$ $\begin{array}{lllllllllllllllllllllllllllllllll}\mathrm{R} & \mathrm{D} & \mathrm{V} & \mathrm{H} & \mathrm{K} & \mathrm{D} & \mathrm{M} & \mathrm{F} & \mathrm{T} & \mathrm{R} & \mathrm{N} & \mathrm{V} & \mathrm{V} & \mathrm{D} & \mathrm{N} & \mathrm{V} & \mathrm{L} & \mathrm{N} & \mathrm{N} & \mathrm{S} & \mathrm{R} & \mathrm{V} & \mathrm{E} & \mathrm{N} & \mathrm{A} & \mathrm{I} & \mathrm{V} & \mathrm{T} & \mathrm{V} & \mathrm{A} & 140\end{array}$ $\begin{array}{llllllllllllllllllllllllllllllll}R & D & V & H & K & G & M & F & A & T & N & V & T & E & N & V & L & N & S & S & R & V & Q & E & A & I & A & E & V & A & 147\end{array}$ $\begin{array}{lllllllllllllllllllllllllllllllll}\text { R } & D & V & H & \text { K } & \text { G } & \text { M } & \text { F } & \text { A } & \text { T } & \text { S } & \text { V } & \text { T } & \text { E } & \text { N } & \text { V } & \text { L } & \text { S } & \text { S } & \text { S } & \text { R } & \text { V } & \text { Q } & \text { E } & \text { A } & \text { I } & \text { A } & \text { E } & \text { V } & \text { A } & 147\end{array}$ $\begin{array}{lllllllllllllllllllllllllllllllll}R & D & V & H & K & G & M & F & A & T & S & I & T & D & N & V & \text { L } & \text { N } & \text { S } & \text { S } & R & \text { V } & \text { Q } & \text { E } & \text { A } & \text { I } & \text { A } & \text { E } & \text { V } & \text { A } & 147\end{array}$ $\begin{array}{llllllllllllllllllllllllllllllll}R & D & V & H & K & G & M & F & A & T & N & V & T & E & N & V & L & N & S & S & R & V & Q & E & A & I & A & E & V & A & 147\end{array}$ $\begin{array}{llllllllllllllllllllllllllllllllll}\text { R } & D & A & H & \text { K } & G & M & F & A & K & N & L & T & E & N & V & \text { L } & \text { N } & \text { S } & \text { S } & R & \text { V } & \text { Q } & \text { K } & \text { A } & \text { I } & \text { V } & \text { D } & \text { E } & \text { A } & 146\end{array}$ $\begin{array}{llllllllllllllllllllllllllllllllll}\mathrm{R} & \mathrm{D} & \mathrm{V} & \mathrm{H} & \mathrm{K} & \mathrm{G} & \mathrm{M} & \mathrm{F} & \mathrm{A} & \mathrm{K} & \mathrm{N} & \mathrm{L} & \mathrm{T} & \mathrm{E} & \mathrm{N} & \mathrm{V} & \mathrm{L} & \mathrm{N} & \mathrm{S} & \mathrm{S} & \mathrm{R} & \mathrm{V} & \mathrm{Q} & \mathrm{K} & \mathrm{A} & \mathrm{I} & \mathrm{V} & \mathrm{D} & \mathrm{E} & \mathrm{A} & 146\end{array}$

$\begin{array}{llllllllllllllllllllllllllllllllll}\text { A } & D & V & D & A & A & G & T & Q & P & G & Q & E & H & K & A & V & R & K & V & K & Q & K & A & R & A & F & \text { L } & \text { Q } & \text { E } & 170\end{array}$

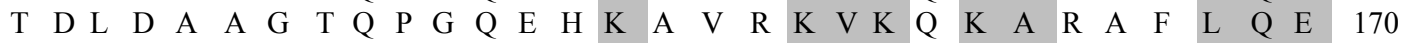
$\begin{array}{llllllllllllllllllllllllllllllll}T & D & L & D & A & A & G & T & Q & P & G & Q & E & H & K & A & V & R & K & V & K & Q & K & A & R & S & F & L & Q & E & 170\end{array}$ $\begin{array}{llllllllllllllllllllllllllllllllll}\text { S } & D & L & D & A & A & G & R & H & P & G & Q & E & H & K & A & I & S & K & V & K & Q & K & A & R & A & F & L & Q & E & 172\end{array}$ $\begin{array}{lllllllllllllllllllllllllllllllll}\text { A } & D & V & D & A & A & S & S & Q & P & G & K & K & Q & K & G & V & S & K & V & K & Q & K & A & R & A & C & L & Q & E & 170\end{array}$ $\begin{array}{llllllllllllllllllllllllllllllll}T & D & L & D & A & A & A & S & Q & P & G & Q & E & H & K & A & V & S & K & V & K & Q & K & A & R & A & F & L & Q & E & 170\end{array}$ $\begin{array}{llllllllllllllllllllllllllllllll}T & D & L & D & A & A & A & S & Q & P & G & Q & E & H & K & A & V & S & K & V & K & Q & K & A & R & A & F & L & Q & E & 170\end{array}$

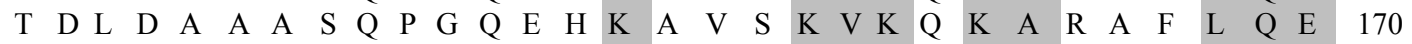
$\begin{array}{llllllllllllllllllllllllllllllll}\text { A } & \text { E } & \text { L } & \text { N } & \text { P } & \text { D } & \text { G } & \text { S } & - & \text { A } & Q & Q & \text { Q } & \text { S } & \text { K } & \text { A } & \text { V } & \text { N } & \text { K } & \text { V } & \text { K } & \text { K } & \text { K } & \text { A } & \text { K } & \text { R } & \text { I } & \text { L } & \text { Q } & \text { E } & 177\end{array}$

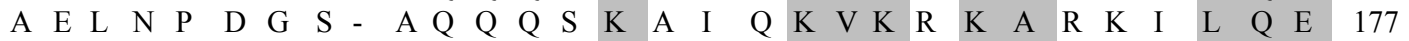

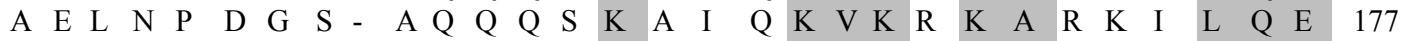
$\begin{array}{llllllllllllllllllllllllllllllll}\text { G } & \text { E } & \text { L } & \text { N } & \text { P } & \text { D } & \text { G } & \text { S } & - & \text { A Q } & \text { Q } & \text { Q } & \text { S } & \text { K } & \text { A } & \text { V } & \text { N } & \text { K } & \text { V } & \text { K } & \text { K } & \text { K } & \text { A } & \text { R } & \text { K } & \text { I } & \text { L } & \text { Q } & \text { E } & 177\end{array}$ $\begin{array}{llllllllllllllllllllllllllllllll}\mathrm{S} & \mathrm{E} & \mathrm{P} & \mathrm{S} & \mathrm{V} & \mathrm{P} & \mathrm{G} & \mathrm{S} & \mathrm{F} & \mathrm{A} & \mathrm{Q} & \mathrm{V} & \mathrm{D} & \mathrm{P} & \mathrm{K} & \mathrm{A} & \mathrm{I} & \mathrm{N} & \mathrm{K} & \mathrm{V} & \mathrm{K} & \mathrm{K} & \mathrm{K} & \mathrm{A} & \mathrm{R} & \mathrm{K} & \mathrm{I} & \mathrm{L} & \mathrm{Q} & \mathrm{E} & 176\end{array}$

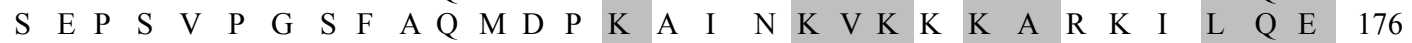

$\begin{array}{lllllllllllllllllllllllllllllll}\text { M V } & \text { A } & \text { N } & \text { I } & \text { S } & \text { P } & \text { A } & \text { F } & \text { I } & \text { R } & \text { L } & \text { T } & G & \text { W } & \text { V } & \text { L } & \text { L } & \text { R } & \text { L } & \text { F } & \text { N } & \text { G } & \text { F } & \text { F } & \text { W } & \text { S } & \text { I } & \text { Q } & \text { I } & 200\end{array}$

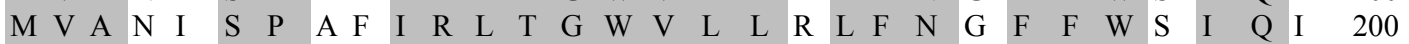
$\begin{array}{llllllllllllllllllllllllllllll}\text { M V V A } & N & \text { I } & \text { S } & \text { P } & \text { A } & F & \text { I } & R & \text { L } & \text { T } & G & \text { W } & \text { V } & \text { L } & \text { L } & \text { R } & \text { L } & F & N & G & F & F & \text { W } & \text { S } & \text { I } & \text { Q } & \text { I } & 200\end{array}$ $\begin{array}{lllllllllllllllllllllllllllllll}\text { M V } & \text { A } & N & \text { I } & \text { S } & \text { P } & \text { A } & F & \text { I } & R & \text { L } & \text { T } & G & \text { W } & \text { V } & \text { L } & \text { L } & \text { R } & \text { L } & F & \text { N } & \text { G } & \text { F } & \text { F } & \text { W } & \text { S } & \text { I } & \text { Q } & \text { I } & 202\end{array}$ $\begin{array}{lllllllllllllllllllllllllllllll}\text { M V } & \text { A } & N & \text { V } & \text { S } & \text { P } & \text { A } & F & \text { I } & R & \text { L } & \text { T } & G & \text { W } & \text { V } & \text { L } & \text { L } & \text { R } & \text { L } & \text { F } & \text { N } & \text { G } & \text { F } & \text { F } & \text { W } & \text { S } & \text { I } & \text { Q } & \text { I } & 200\end{array}$ $\begin{array}{lllllllllllllllllllllllllllll}\text { M V } & \text { A } & N & \text { I } & \text { S } & \text { P } & \text { A A F } & \text { I } & R & \text { L } & \text { T } & G & \text { W } & \text { V } & \text { L } & \text { L } & \text { R } & \text { L } & F & \text { N } & \text { G } & \text { F } & \text { F } & \text { W } & \text { S } & \text { I } & \text { Q } & \text { I } & 200\end{array}$ $\begin{array}{llllllllllllllllllllllllllllll}\text { M V } & \text { A } & N & I & S & P & A & F & I & R & L & T & G & \text { W } & \text { V } & \text { L } & \text { L } & R & \text { L } & F & N & G & F & F & \text { W } & \text { S } & \text { I } & \text { Q } & \text { I } & 200\end{array}$ $\begin{array}{lllllllllllllllllllllllllllllll}\text { M V V A } & N & \text { I } & \text { S } & \text { P } & \text { A } & F & \text { I } & R & \text { L } & \text { T } & G & \text { W } & \text { V } & \text { L } & \text { L } & \text { R } & \text { L } & \text { F } & \text { N } & \text { G } & \text { F } & \text { F } & \text { W } & \text { S } & \text { I } & \text { Q } & \text { I } & 200\end{array}$ $\begin{array}{lllllllllllllllllllllllllllllll}\text { M V } & \text { A } & \text { T } & \text { V } & \text { S } & \text { P } & \text { A } & \text { M } & \text { I } & \text { R } & \text { L } & \text { T } & \text { G } & \text { W } & \text { V } & \text { L } & \text { L } & \text { K } & \text { L } & \text { F } & \text { N } & \text { S } & \text { F } & \text { F } & \text { W } & \text { N } & \text { I } & \text { Q } & \text { I } & 207\end{array}$ $\begin{array}{llllllllllllllllllllllllllllllll}\text { M V } & \text { A } & \text { T } & \text { V } & \text { S } & \text { P } & \text { G } & \text { M } & \text { I } & \text { R } & \text { L } & \text { T } & \text { G } & \text { W } & \text { V } & \text { L } & \text { L } & \text { K } & \text { L } & \text { F } & \text { N } & \text { S } & \text { F } & \text { F } & \text { W } & \text { N } & \text { I } & \text { Q } & \text { I } & 207\end{array}$ $\begin{array}{lllllllllllllllllllllllllllllll}\text { M V V A } & \text { T } & \text { V } & \text { S } & \text { P } & \text { G } & \text { M } & \text { I } & \text { R } & \text { L } & \text { T } & \text { G } & \text { W } & \text { V } & \text { L } & \text { L } & \text { K } & \text { L } & \text { F } & \text { N } & \text { S } & \text { F } & \text { F } & \text { W } & \text { N } & \text { I } & \text { Q } & \text { I } & 207\end{array}$ $\begin{array}{lllllllllllllllllllllllllllllll}\text { M V V A } & \text { T } & \text { V } & \text { S } & \text { P } & \text { A } & \text { M } & \text { I } & \text { R } & \text { L } & \text { T } & G & \text { W } & \text { V } & \text { L } & \text { L } & \text { K } & \text { L } & \text { F } & \text { N } & \text { S } & \text { F } & \text { F } & \text { W } & \text { N } & \text { I } & \text { Q } & \text { I } & 207\end{array}$ $\begin{array}{llllllllllllllllllllllllllllll}\text { M V } & \text { A } & N & \text { V } & \text { S } & \text { P } & \text { A } & \text { L } & \text { I } & \text { R } & \text { L } & \text { T } & G & \text { W } & \text { V } & \text { L } & \text { L } & \text { K } & \text { L } & \text { F } & \text { N } & \text { S } & \text { F } & \text { F } & \text { W } & \text { N } & \text { I } & \text { Q } & \text { V } & 206\end{array}$

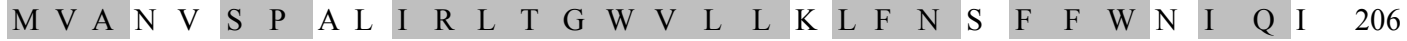

$\begin{array}{lllllllllllllllllllllllllllllllll} & H & K & G & Q & L & E & M & V & K & K & A & A & T & E & Q & N & V & P & M & V & F & \text { L } & \text { P } & \text { V } & \text { H } & \text { K } & \text { S } & \text { H } & \text { I } & \text { D } & 230\end{array}$ $\begin{array}{llllllllllllllllllllllllllllllll}\text { H } & \text { K } & G & Q & L & E & M & V & K & K & A & A & T & E & Q & N & V & P & M & V & F & \text { L } & \text { P } & \text { V } & \text { H } & \text { K } & \text { S } & \text { H } & \text { I } & \text { D } & 230\end{array}$

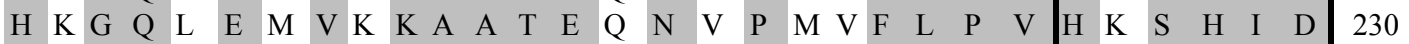
$\begin{array}{lllllllllllllllllllllllllllllll}H & K & G & Q & L & E & M & V & K & K & A & A & T & E & Q & N & V & P & M & V & F & L & P & V & H & K & S & H & \text { I } & \text { D } & 232\end{array}$ $\begin{array}{lllllllllllllllllllllllllllllllll}\text { H } & \text { K } & G & Q & \text { L } & \text { E } & \text { M } & \text { V } & \text { K } & \text { K } & \text { A } & \text { A } & \text { T } & \text { E } & \text { Q } & \text { N } & \text { V } & \text { P } & \text { M } & \text { I } & \text { F } & \text { L } & \text { P } & \text { V } & \text { H } & \text { K } & \text { S } & \text { H } & \text { I } & \text { D } & 230\end{array}$

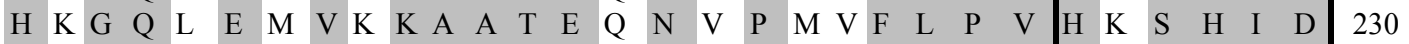

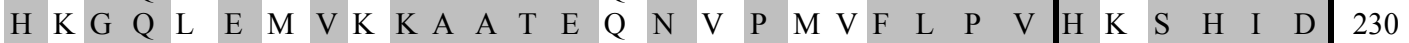
$\begin{array}{lllllllllllllllllllllllllllllll}\text { H } & K & G & Q & L & E & M & V & K & K & A & A & T & E & Q & N & V & P & M & V & F & L & P & V & H & K & S & H & \text { I } & \text { D } & 230\end{array}$ $\begin{array}{lllllllllllllllllllllllllllllll}\text { H } & \text { K } & G & \text { Q } & \text { L } & \text { E } & \text { M } & \text { V } & \text { K } & - & \text { A } & \text { A } & \text { T } & \text { E } & \text { T } & \text { N } & \text { L } & \text { P } & \text { L } & \text { L } & \text { F } & \text { L } & \text { P } & \text { V } & \text { H } & \text { R } & \text { S } & \text { H } & \text { I } & \text { D } & 236\end{array}$

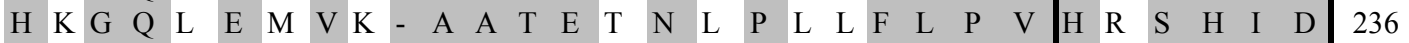
$\begin{array}{lllllllllllllllllllllllllllllll}\text { H } & \text { K G } & \text { Q } & \text { L } & \text { E } & \text { M } & \text { V } & \text { K } & - & \text { A } & \text { A } & \text { T } & \text { E } & \text { T } & \text { N } & \text { L } & \text { P } & \text { L } & \text { L } & \text { F } & \text { L } & \text { P } & \text { V } & \text { H } & \text { R } & \text { S } & \text { H } & \text { I } & \text { D } & 236\end{array}$ $\begin{array}{llllllllllllllllllllllllllllllll}\text { H } & \text { K G } & \text { Q } & \text { L } & \text { E } & \text { M } & \text { V } & \text { K } & - & \text { A } & \text { A } & \text { T } & \text { E } & \text { M } & \text { N } & \text { L } & \text { P } & \text { L } & \text { I } & \text { F } & \text { L } & \text { P } & \text { V } & \text { H } & \text { R } & \text { S } & \text { H } & \text { I } & \text { D } & 236\end{array}$

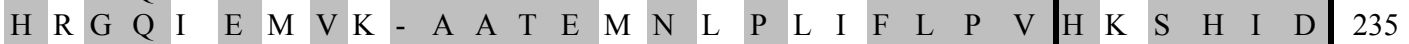
$\begin{array}{lllllllllllllllllllllllllllllll}\text { H } & \text { R } & G & \text { Q } & \text { I } & \text { E } & M & \text { V } & \text { K } & - & \text { A } & \text { A } & \text { T } & \text { E } & \text { M } & \text { N } & \text { L } & \text { P } & \text { L } & \text { I } & \text { F } & \text { L } & \text { P } & \text { V } & \text { H } & \text { K } & \text { S } & \text { H } & \text { I } & \text { D } & 235\end{array}$

$\begin{array}{lllllllllllllllllllllllllllllllll}\text { Y } & \text { L } & \text { L } & \text { I } & \text { T } & \text { L } & \text { I } & \text { L } & \text { F } & \text { C } & \text { H } & \text { N } & \text { I } & \text { K } & \text { A } & \text { P } & \text { H } & \text { I } & \text { A } & \text { A } & \text { G } & \text { N } & \text { N } & \text { L } & \text { S } & \text { I } & \text { P } & \text { I } & \text { L } & \text { S } & 260\end{array}$

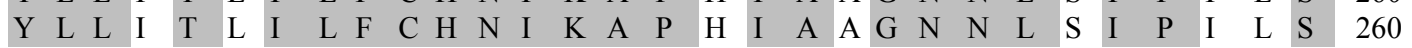


E. maclovinus

G. aculeatus

T. rubripes

M. zebra

P. nyererei

O. niloticus

H. sapiens

M. musculus

$R$. norvegicus

B. taurus

M. gallopavo

G. gallus

N. coriiceps

C. aceratus

E. maclovinus

G. aculeatus

T. rubripes

M. zebra

P. nyererei

O. niloticus

H. sapiens

M. musculus

$R$. norvegicus

B. taurus

M. gallopavo

G. gallus

N. coriiceps

C. aceratus

E. maclovinus

G. aculeatus

T. rubripes

M. zebra

P. nyererei

$O$. niloticus

$H$. sapiens

M. musculus

$R$. norvegicus

B. taurus

M. gallopavo

G. gallus

N. coriiceps

C. aceratus

E. maclovinus

G. aculeatus

T. rubripes

M. zebra

$P$. nyererei

$O$. niloticus

H. sapiens

M. musculus

$R$. norvegicus

B. taurus

M. gallopavo

G. gallus

$N$. coriiceps

C. aceratus $\begin{array}{llllllllllllllllllllllllllllllllll}\text { Y } & \text { L } & \text { L } & \text { I } & \text { T } & \text { L } & \text { I } & \text { L } & \text { F } & \text { C } & \text { H } & \text { N } & \text { I } & \text { K } & \text { A } & \text { P } & \text { H } & \text { I } & \text { A } & \text { A } & \text { G } & \text { N } & \text { N } & \text { L } & \text { S } & \text { I } & \text { P } & \text { I } & \text { L } & \text { S } & 260\end{array}$ $\begin{array}{lllllllllllllllllllllllllllllll}\text { Y } & \text { L } & \text { L } & \text { I } & \text { T } & \text { L } & \text { I } & \text { L } & \text { F } & \text { C } & \text { H } & \text { N } & \text { I } & \text { K } & \text { A } & \text { P } & \text { H } & \text { I } & \text { A } & \text { A } & \text { G } & \text { N } & \text { N } & \text { L } & \text { S } & \text { I } & \text { P } & \text { I } & \text { L } & \text { S } & 262\end{array}$

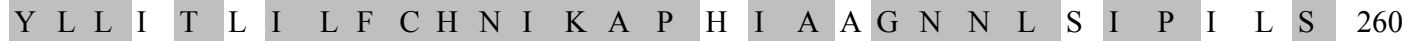

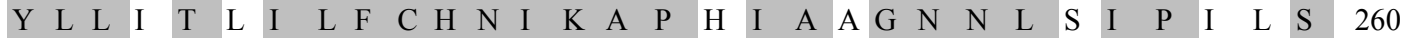

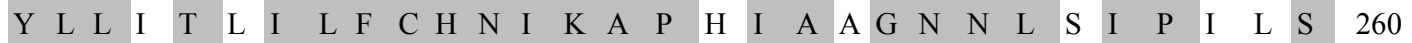
$\begin{array}{llllllllllllllllllllllllllllllll}\text { Y } & \text { L } & \text { L } & \text { I } & \text { T } & \text { L } & \text { I } & \text { L } & \text { F } & \text { C } & \text { H } & \text { N } & \text { I } & \text { K } & \text { A } & \text { P } & \text { H } & \text { I } & \text { A } & \text { A } & \text { G } & \text { N } & \text { N } & \text { L } & \text { S } & \text { I } & \text { P } & \text { I } & \text { L } & \text { S } & 260\end{array}$ $\begin{array}{llllllllllllllllllllllllllllllll}\text { Y } & \text { L } & \text { L } & \text { L } & \text { T } & \text { F } & \text { I } & \text { L } & \text { F } & \text { C } & \text { H } & \text { N } & \text { I } & \text { K } & \text { A } & \text { P } & \text { Y } & \text { I } & \text { A } & \text { S } & G & \text { N } & \text { N } & \text { L } & \text { N } & \text { I } & \text { P } & \text { I } & \text { F } & \text { S } & 266\end{array}$ $\begin{array}{lllllllllllllllllllllllllllllllll}\text { Y } & \text { L } & \text { L } & \text { L } & \text { T } & \text { F } & \text { I } & \text { L } & \text { F } & \text { C } & \text { H } & \text { N } & \text { I } & \text { K } & \text { A } & \text { P } & \text { Y } & \text { I } & \text { A } & \text { S } & \text { G } & \text { N } & \text { N } & \text { L } & \text { N } & \text { I } & \text { P } & \text { V } & \text { F } & \text { S } & 266\end{array}$ $\begin{array}{llllllllllllllllllllllllllllllll}\text { Y } & \text { L } & \text { L } & \text { L } & \text { T } & \text { F } & \text { I } & \text { L } & \text { F } & \text { C } & \text { H } & \text { N } & \text { I } & \text { K } & \text { A } & \text { P } & \text { Y } & \text { I } & \text { A } & \text { S } & \text { G } & \text { N } & \text { N } & \text { L } & \text { N } & \text { I } & \text { P } & \text { I } & \text { F } & \text { S } & 266\end{array}$ $\begin{array}{llllllllllllllllllllllllllllllll}\text { Y } & \text { L } & \text { L } & \text { L } & \text { T } & \text { F } & \text { I } & \text { L } & \text { F } & \text { C } & \text { H } & \text { N } & \text { I } & \text { K } & \text { A } & \text { P } & \text { Y } & \text { I } & \text { A } & \text { S } & \text { G } & \text { N } & \text { N } & \text { L } & \text { N } & \text { I } & \text { P } & \text { I } & \text { F } & \text { S } & 266\end{array}$

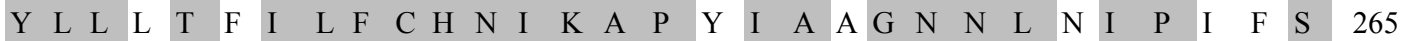

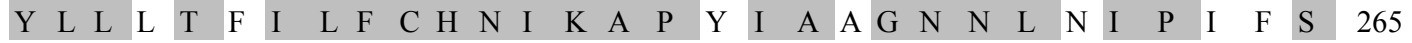

\begin{tabular}{llllllll|llllllllllllllllllllllllll}
$\mathrm{T}$ & $\mathrm{L}$ & $\mathrm{I}$ & $\mathrm{R}$ & $\mathrm{K}$ & $\mathrm{L}$ & $\mathrm{G}$ & $\mathrm{G}$ & $\mathrm{F}$ & $\mathrm{F}$ & $\mathrm{I}$ & $\mathrm{R}$ & $\mathrm{R}$ & $\mathrm{R}$ & $\mathrm{M}$ & $\mathrm{D}$ & $\mathrm{E}$ & $\mathrm{T}$ & $\mathrm{G}$ & $\mathrm{D}$ & - & $\mathrm{K}$ & $\mathrm{K}$ & $\mathrm{D}$ & $\mathrm{V}$ & $\mathrm{L}$ & $\mathrm{Y}$ & $\mathrm{R}$ & $\mathrm{S}$ & $\mathrm{L}$ & 289
\end{tabular} \begin{tabular}{llllllll|llll|llllllllllllllllllllll} 
T & L & I & R & K & L & $G$ & $G$ & $F$ & F & I & R & R & R & M & D & E & T & G & D & - & K & K & D & V & L & Y & R & S & L & 289
\end{tabular} \begin{tabular}{llllllll|llll|llllllllllllllllllllll} 
T & L & I & R & K & L & $G$ & $G$ & F & F & I & R & R & K & M & E & E & T & G & D & - & K & K & D & V & L & Y & R & S & L & 289
\end{tabular} \begin{tabular}{llllllll|llll|llllllllllllllllllll}
$\mathrm{T}$ & $\mathrm{L}$ & $\mathrm{I}$ & $\mathrm{R}$ & $\mathrm{K}$ & $\mathrm{L}$ & $\mathrm{G}$ & $\mathrm{G}$ & $\mathrm{F}$ & $\mathrm{F}$ & $\mathrm{I}$ & $\mathrm{R}$ & $\mathrm{R}$ & $\mathrm{R}$ & $\mathrm{M}$ & $\mathrm{E}$ & $\mathrm{E}$ & $\mathrm{T}$ & $\mathrm{G}$ & $\mathrm{D}$ & $\mathrm{G}$ & $\mathrm{K}$ & $\mathrm{R}$ & $\mathrm{D}$ & $\mathrm{I}$ & $\mathrm{L}$ & $\mathrm{Y}$ & $\mathrm{R}$ & $\mathrm{S}$ & $\mathrm{L}$ & 292
\end{tabular} \begin{tabular}{llllllll|llll|llllllllllllllllllllll} 
T & L & I & R & K & L & $G$ & $G$ & $F$ & F & I & R & R & K & M & E & E & T & E & D & G & K & K & D & I & L & Y & R & S & L & 290
\end{tabular} \begin{tabular}{llllllll|llllllllllllllllllllllllll} 
T & L & I & R & K & L & $G$ & $G$ & F & F & I & R & R & R & M & E & E & S & A & D & G & K & K & D & I & L & Y & R & S & L & 290
\end{tabular} \begin{tabular}{llllllll|llll|llllllllllllllllllll} 
T & L & I & R & $K$ & L & $G$ & $G$ & $F$ & $F$ & $I$ & $R$ & $R$ & $R$ & $M$ & $E$ & $E$ & S & $A$ & $D$ & $G$ & $K$ & $K$ & $D$ & $I$ & L & Y & R & S & L & 290
\end{tabular}

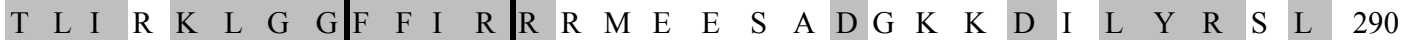

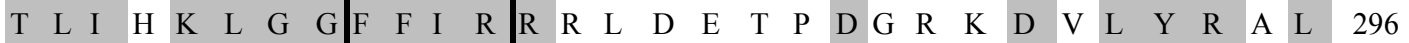
\begin{tabular}{llllllll|llll|lllllllllllllllllllll} 
T & L & I & H & K & L & $G$ & $G$ & $F$ & $F$ & I & R & R & R & L & D & E & T & P & D & $G$ & $R$ & K & D & I & L & Y & R & A & L & 296
\end{tabular} \begin{tabular}{llllllll|llll|llllllllllllllllllll} 
T & L & I & H & K & L & $G$ & $G$ & $F$ & $F$ & $I$ & $R$ & $R$ & $R$ & $L$ & $D$ & $E$ & T & P & D & $G$ & $R$ & K & D & I & L & Y & R & A & L & 296
\end{tabular} \begin{tabular}{llllllll|llll|lllllllllllllllllllll} 
T & L & I & H & K & L & $G$ & G & F & F & I & R & R & R & L & D & E & T & P & D & G & R & K & D & I & L & Y & R & A & L & 296
\end{tabular} \begin{tabular}{llllllll|llll|lllllllllllllllllllll} 
T & L & I & R & K & L & G & G & F & F & I & R & R & K & L & D & Q & S & P & D & G & Q & K & D & F & L & Y & R & A & L & 295
\end{tabular} \begin{tabular}{llllllll|llll|lllllllllllllllllllllll} 
T & L & I & R & K & L & $G$ & $G$ & F & F & I & R & R & K & L & D & Q & S & S & D & G & R & K & D & F & L & Y & R & A & L & 295
\end{tabular}

$\begin{array}{lllllllllllllllllllllllllllllllll}\text { L } & H & A & Y & T & E & E & L & L & R & Q & Q & Q & F & L & E & I & Y & L & E & G & T & R & S & R & S & G & K & P & S & 319\end{array}$

\begin{tabular}{lllllllllllllllll|llllllllllllllll} 
L & $H$ & $A$ & $Y$ & $T$ & $E$ & $E$ & $L$ & $L$ & $R$ & $Q$ & $Q$ & $Q$ & $F$ & $L$ & $E$ & $I$ & $Y$ & $L$ & $E$ & $G$ & $T$ & $R$ & $S$ & $R$ & $S$ & $G$ & $K$ & $P$ & $S$ & 319
\end{tabular}

\begin{tabular}{lllllllllllllllll|llllllllllllllll} 
L & $H$ & $A$ & $Y$ & $T$ & $E$ & $E$ & $L$ & $L$ & $R$ & $Q$ & $Q$ & $Q$ & $F$ & $L$ & $E$ & $I$ & $Y$ & $L$ & $E$ & $G$ & $T$ & $R$ & $S$ & $R$ & $S$ & $G$ & $K$ & $P$ & $S$ & 319
\end{tabular}

$\begin{array}{llllllllllllllllllllllllllllllll}\text { L } & H & A & Y & T & E & E & L & L & R & Q & H & Q & F & L & E & V & Y & L & E & G & T & R & S & R & S & G & K & P & S & 322\end{array}$

\begin{tabular}{lllllllllllllllllllllll|llllllll} 
L & Y & $A$ & Y & T & E & E & L & L & C & Q & Q & Q & F & L & E & V & Y & L & E & $G$ & T & R & S & R & S & G & K & P & S & 320
\end{tabular}

$\begin{array}{lllllllllllllllllllllllllllllllll}\mathrm{L} & \mathrm{H} & \text { A } & \mathrm{Y} & \mathrm{T} & \mathrm{E} & \mathrm{E} & \mathrm{L} & \mathrm{L} & \mathrm{R} & \mathrm{Q} & \mathrm{Q} & \mathrm{Q} & \mathrm{F} & \mathrm{L} & \mathrm{E} & \mathrm{V} & \mathrm{F} & \mathrm{L} & \mathrm{E} & \mathrm{G} & \mathrm{T} & \mathrm{R} & \mathrm{S} & \mathrm{R} & \mathrm{S} & \mathrm{G} & \mathrm{K} & \mathrm{P} & \mathrm{S} & 320\end{array}$

\begin{tabular}{lllllllllllllllll|lllllllllllllll} 
L & $H$ & $A$ & $Y$ & $T$ & $E$ & $E$ & $L$ & $L$ & $R$ & $Q$ & $Q$ & $Q$ & $F$ & $L$ & $E$ & $V$ & $F$ & $L$ & $E$ & $G$ & $T$ & $R$ & $S$ & $R$ & $S$ & $G$ & $K$ & $P$ & $S$ & 320
\end{tabular}

\begin{tabular}{lllllllllllllllll|llllllllllllllll} 
L & $H$ & $A$ & $Y$ & $T$ & $E$ & $E$ & $L$ & $L$ & $R$ & $Q$ & $Q$ & $Q$ & $F$ & $L$ & $E$ & $V$ & $F$ & $L$ & $E$ & $G$ & $T$ & $R$ & $S$ & $R$ & $S$ & $G$ & $K$ & $P$ & $S$ & 320
\end{tabular}

\begin{tabular}{lllllllllllllllll|lllllllllllllll} 
L & H & $G$ & $H$ & I & V & E & L & L & R & Q & Q & Q & F & L & E & I & F & L & E & $G$ & T & R & S & R & S & G & K & T & S & 326
\end{tabular}

\begin{tabular}{lllllllllllllllll|llllllllllllllll}
$\mathrm{L}$ & $\mathrm{H}$ & $\mathrm{G}$ & $\mathrm{H}$ & $\mathrm{V}$ & $\mathrm{V}$ & $\mathrm{E}$ & $\mathrm{L}$ & $\mathrm{L}$ & $\mathrm{R}$ & $\mathrm{Q}$ & $\mathrm{Q}$ & $\mathrm{Q}$ & $\mathrm{F}$ & $\mathrm{L}$ & $\mathrm{E}$ & $\mathrm{I}$ & $\mathrm{F}$ & $\mathrm{L}$ & $\mathrm{E}$ & $\mathrm{G}$ & $\mathrm{T}$ & $\mathrm{R}$ & $\mathrm{S}$ & $\mathrm{R}$ & $\mathrm{S}$ & $\mathrm{G}$ & $\mathrm{K}$ & $\mathrm{T}$ & $\mathrm{S}$ & 326
\end{tabular}

\begin{tabular}{lllllllllllllllll|llllll|llllllll} 
L & $H$ & $G$ & $H$ & $I$ & V & E & L & L & R & Q & Q & Q & F & L & E & I & F & L & E & $G$ & T & R & S & R & S & $G$ & K & T & S & 326
\end{tabular}

\begin{tabular}{lllllllllllllllll|llllll|lllllllll} 
L & $H$ & $G$ & $H$ & $I$ & V & E & L & L & R & $Q$ & $Q$ & $Q$ & $F$ & L & E & I & F & L & E & $G$ & T & R & S & R & S & G & K & I & S & 326
\end{tabular}

\begin{tabular}{lllllllllllllllll|llllll|llllllll} 
L & Y & V & H & I & E & E & L & L & R & Q & Q & Q & F & L & E & I & F & L & E & $G$ & T & R & S & R & S & G & K & T & S & 325
\end{tabular}

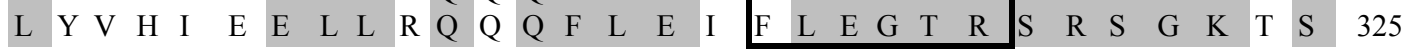

\begin{tabular}{lllllllllllllllllllll|llllllllllll} 
T & A & R & A & $G$ & M & L & S & I & V & V & D & T & M & W & T & G & S & I & P & D & V & L & V & V & P & V & G & I & S & 349
\end{tabular} \begin{tabular}{lllllllllllllllllllll|llllllllllll}
$T$ & A & $R$ & $A$ & $G$ & $M$ & $L$ & $S$ & $I$ & $V$ & $V$ & $D$ & $T$ & $M$ & W & $T$ & $G$ & S & I & P & D & V & L & V & V & P & V & G & I & S & 349
\end{tabular}

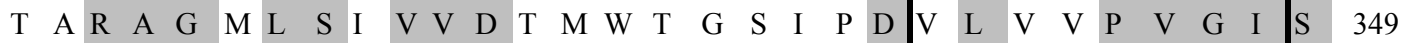

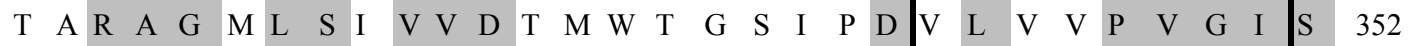

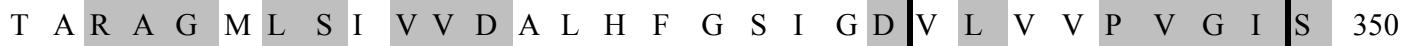

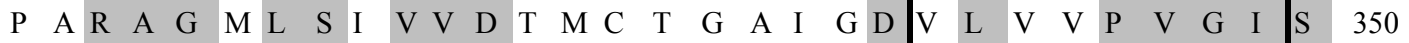

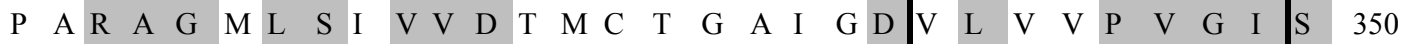

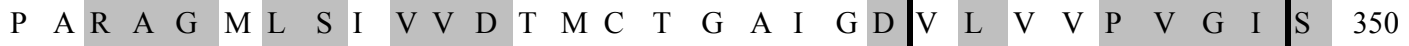

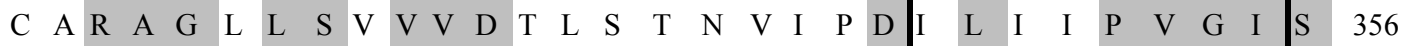

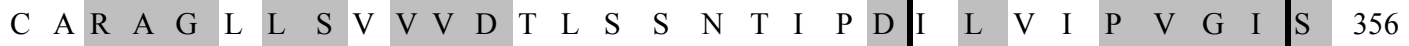

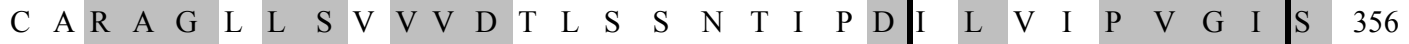
\begin{tabular}{lllllllllllllllllllll|lllllllllll} 
C & A & R & A & $G$ & L & L & S & V & V & V & D & T & L & S & T & N & T & I & P & D & I & L & I & I & P & V & G & I & S & 356
\end{tabular} \begin{tabular}{lllllllllllllllllllll|llllllllllll} 
G & P & R & A & $G$ & L & L & S & V & V & V & D & A & L & F & S & N & A & T & P & D & V & L & I & I & P & V & G & I & S & 355
\end{tabular} $\begin{array}{lllllllllllllllllllllllllllllllll}\text { S } & \text { P } & \text { R } & \text { A } & G & \text { L } & \text { L } & \text { S } & \text { V } & \text { V } & \text { V } & \text { D } & \text { A } & \text { L } & \text { F } & \text { S } & \text { N } & \text { A } & \text { T } & \text { P } & \text { D } & \text { V } & \text { L } & \text { I } & \text { I } & \text { P } & \text { V } & \text { G } & \text { I } & \text { S } & 355\end{array}$

$\begin{array}{llllllllllllllllllllllllllllllllll}\text { Y } & \text { D } & R & \text { I } & \text { L } & \text { E } & G & \text { N } & \text { Y } & \text { N } & \text { S } & \text { E } & \text { Q } & \text { L } & \text { G } & \text { K } & \text { P } & \text { K } & \text { K } & \text { N } & \text { E } & \text { S } & \text { L } & \text { W } & \text { G } & \text { I } & \text { A } & \text { C } & \text { G } & \text { V } & 379\end{array}$

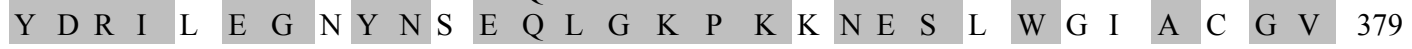


E. maclovinus

G. aculeatus

T. rubripes

M. zebra

P. nyererei

O. niloticus

H. sapiens

M. musculus

$R$. norvegicus

B. taurus

M. gallopavo

G. gallus

N. coriiceps

C. aceratus

E. maclovinus

G. aculeatus

T. rubripes

M. zebra

P. nyererei

O. niloticus

H. sapiens

M. musculus

$R$. norvegicus

B. taurus

M. gallopavo

G. gallus

$N$. coriiceps

C. aceratus

E. maclovinus

G. aculeatus

T. rubripes

M. zebra

P. nyererei

$O$. niloticus

H. sapiens

M. musculus

$R$. norvegicus

B. taurus

M. gallopavo

G. gallus

N. coriiceps

C. aceratus

E. maclovinus

G. aculeatus

T. rubripes

M. zebra

P. nyererei

O. niloticus

H. sapiens

M. musculus

$R$. norvegicus

B. taurus

M. gallopavo

G. gallus

N. coriiceps $\begin{array}{lllllllllllllllllllllllllllllllll}\text { Y } & \text { D } & R & \text { I } & \text { L } & \text { E } & G & \text { N } & \text { Y } & \text { N } & \text { S } & \text { E } & \text { Q } & \text { L } & \text { G } & \text { K } & \text { P } & \text { K } & \text { K } & \text { N } & \text { E } & \text { S } & \text { L } & \text { W } & \text { G } & \text { I } & \text { A } & \text { C } & \text { G } & \text { V } & 379\end{array}$

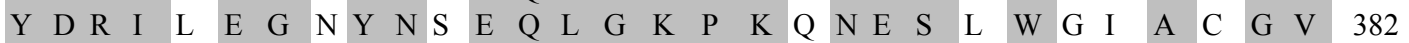
$\begin{array}{llllllllllllllllllllllllllllllll}\text { Y } & D & R & \text { I } & \text { I } & \text { E } & G & \text { N } & \text { Y } & \text { N } & \text { S } & \text { E } & \text { Q } & \text { L } & G & \text { K } & \text { P } & \text { K } & \text { K } & \text { N } & \text { E } & \text { S } & \text { W } & \text { W } & \text { G } & \text { I } & \text { A } & \text { C } & G & \text { V } & 380\end{array}$ $\begin{array}{llllllllllllllllllllllllllllllll}Y & D & R & I & I & E & G & N & Y & N & S & E & Q & L & G & K & P & K & K & N & E & S & \text { W } & \text { W } & \text { G } & \text { I } & \text { A } & \text { C } & G & \text { V } & 380\end{array}$

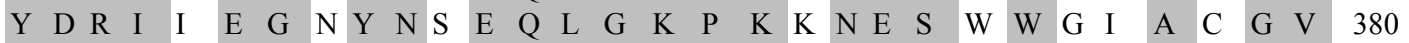
$\begin{array}{llllllllllllllllllllllllllllllll}\text { Y } & D & R & I & I & E & G & N & Y & N & S & E & Q & L & G & K & P & K & K & N & E & S & \text { W } & \text { W } & G & \text { I } & \text { A } & C & G & V & 380\end{array}$ $\begin{array}{lllllllllllllllllllllllllllllllll}Y & D & R & I & I & E & G & H & Y & N & G & E & Q & L & G & K & P & K & K & N & E & S & L & \text { W } & \text { S } & \text { V } & \text { A } & R & G & V & 386\end{array}$ $\begin{array}{llllllllllllllllllllllllllllllll}\text { Y } & D & R & I & \text { I } & \text { E } & G & \text { H } & \text { Y } & \text { N } & G & \text { E } & \text { Q } & \text { L } & G & \text { K } & \text { P } & \text { K } & \text { K } & \text { N } & \text { E } & \text { S } & \text { L } & \text { W } & \text { S } & \text { V } & \text { A } & \text { R } & G & \text { V } & 386\end{array}$ $\begin{array}{lllllllllllllllllllllllllllllllll}Y & D & R & I & I & E & G & H & Y & N & G & E & Q & L & G & K & P & K & K & N & E & S & L & \text { W } & \text { S } & \text { V } & \text { A } & \text { R } & G & \text { V } & 386\end{array}$

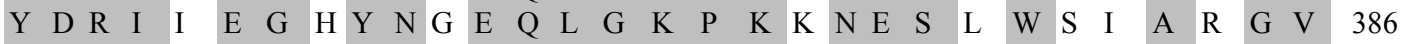

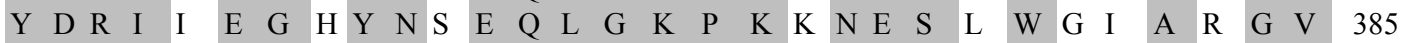

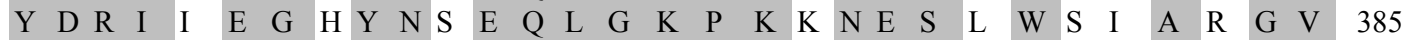

$\begin{array}{lllllllllllllllllllllllllllllllll}\text { F } & \text { R } & \text { M } & \text { L } & \text { R } & \text { K } & \text { N } & \text { Y } & G & \text { C } & \text { V } & \text { R } & \text { V } & \text { D } & \text { F } & \text { N } & \text { Q } & \text { P } & \text { F } & \text { S } & \text { L } & \text { K } & \text { E } & \text { Y } & \text { L } & \text { D } & \text { S } & \text { Q } & \text { R } & \text { N } & 409\end{array}$ $\begin{array}{llllllllllllllllllllllllllllllllll}\text { F } & \text { R } & M & L & R & K & N & Y & G & C & V & R & V & D & F & N & Q & P & F & S & L & K & E & Y & L & D & S & Q & R & N & 409\end{array}$ $\begin{array}{lllllllllllllllllllllllllllllll}\text { F } & R & M & L & R & K & N & Y & G & C & V & R & V & D & F & N & Q & P & F & S & L & K & E & Y & L & D & S & Q & R & N & 409\end{array}$ $\begin{array}{llllllllllllllllllllllllllllllll}\text { F } & R & M & L & R & K & N & Y & G & C & V & R & V & D & F & N & Q & P & F & S & L & K & E & Y & L & D & S & Q & R & N & 412\end{array}$ $\begin{array}{lllllllllllllllllllllllllllllll}\text { C } & R & M & L & R & K & N & Y & G & C & V & R & V & D & F & N & Q & P & F & S & L & K & E & Y & L & D & T & Q & R & S & 410\end{array}$ $\begin{array}{llllllllllllllllllllllllllllllll}\text { F } & R & M & L & R & K & N & Y & G & C & V & R & V & D & F & N & Q & P & F & S & L & K & E & Y & L & D & T & Q & R & S & 410\end{array}$ $\begin{array}{lllllllllllllllllllllllllllllll}\text { F } & \text { R } & \text { M } & \text { L } & \text { R } & \text { K } & \text { N } & \text { Y } & G & C & \text { V } & \text { R } & \text { V } & \text { D } & \text { F } & \text { N } & \text { Q } & \text { P } & \text { F } & \text { S } & \text { L } & \text { K } & \text { E } & \text { Y } & \text { L } & \text { D } & \text { T } & \text { Q } & \text { R } & \text { S } & 410\end{array}$ $\begin{array}{llllllllllllllllllllllllllllllll}\text { F } & R & M & L & R & K & N & Y & G & C & V & R & V & D & F & N & Q & P & F & S & L & K & E & Y & L & D & T & Q & R & S & 410\end{array}$ $\begin{array}{lllllllllllllllllllllllllllllll}\text { I } & R & M & L & R & K & N & Y & G & C & V & R & V & D & F & A & Q & P & F & S & L & K & E & Y & L & E & S & Q & S & Q & 416\end{array}$ $\begin{array}{llllllllllllllllllllllllllllllll}\text { I } & R & M & L & R & K & N & Y & G & Y & V & R & V & D & F & A & Q & P & F & \text { S } & \text { L } & \text { K } & \text { E } & \text { Y } & \text { L } & \text { E } & \text { G } & \text { Q } & \text { S } & \text { Q } & 416\end{array}$ $\begin{array}{lllllllllllllllllllllllllllllll}\text { I } & R & M & L & R & K & N & Y & G & Y & V & R & V & D & F & A & Q & P & F & S & L & K & E & Y & L & E & G & Q & S & Q & 416\end{array}$ $\begin{array}{lllllllllllllllllllllllllllllll}\text { I } & R & M & L & R & K & N & Y & G & C & V & R & V & D & F & A & Q & P & F & S & L & K & E & Y & L & E & S & Q & S & Q & 416\end{array}$ $\begin{array}{lllllllllllllllllllllllllllllll}\text { F } & \text { R } & \text { M } & \text { L } & \text { R } & \text { K } & \text { N } & \text { Y } & G & \text { C } & \text { V } & \text { R } & \text { V } & \text { D } & \text { F } & \text { A } & \text { Q } & \text { P } & \text { F } & \text { S } & \text { L } & \text { K } & \text { E } & \text { Y } & \text { V } & \text { N } & \text { S } & \text { Q } & \text { S } & \text { Q } & 415\end{array}$

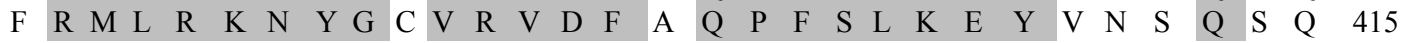

$\begin{array}{lllllllllllllllllllllllllllllllll}\text { R } & \text { H I } & \text { P } & \text { P } & \text { A } & \text { V } & \text { S } & \text { L } & \text { E } & \text { H } & \text { T } & \text { L } & \text { M } & \text { P } & \text { I } & \text { I } & \text { I } & \text { S } & \text { A } & \text { Q } & \text { P } & \text { D } & \text { A } & \text { Q } & \text { L } & \text { F } & \text { E } & \text { G } & \text { Q } & 439\end{array}$ $\begin{array}{llllllllllllllllllllllllllllllll}\mathrm{R} & \mathrm{H} & \mathrm{I} & \mathrm{P} & \mathrm{P} & \mathrm{A} & \mathrm{V} & \mathrm{S} & \mathrm{L} & \mathrm{E} & \mathrm{Q} & \mathrm{T} & \mathrm{L} & \mathrm{M} & \mathrm{P} & \mathrm{I} & \mathrm{I} & \mathrm{I} & \mathrm{S} & \mathrm{A} & \mathrm{Q} & \mathrm{P} & \mathrm{D} & \mathrm{A} & \mathrm{Q} & \mathrm{L} & \mathrm{F} & \mathrm{E} & \mathrm{G} & \mathrm{Q} & 439\end{array}$ $\begin{array}{lllllllllllllllllllllllllllllll}\text { R } & H & I & P & P & \text { S } & \text { V } & \text { S } & \text { L } & \text { E } & \text { H } & \text { T } & \text { L } & \text { M } & \text { P } & \text { I } & \text { I } & \text { I } & \text { S } & \text { A } & \text { Q } & \text { P } & \text { D } & \text { A } & \text { Q } & \text { L } & \text { F } & \text { E } & \text { G } & \text { Q } & 439\end{array}$ $\begin{array}{lllllllllllllllllllllllllllllll}\text { R } & H & \text { I } & \text { P } & \text { P } & \text { S } & \text { V } & \text { S } & \text { L } & \text { E } & \text { Q } & \text { T } & \text { L } & \text { M } & \text { P } & \text { I } & \text { I } & \text { I } & \text { S } & \text { A } & \text { Q } & \text { P } & \text { D } & \text { A } & \text { Q } & \text { L } & \text { F } & \text { E } & \text { G } & \text { Q } & 442\end{array}$

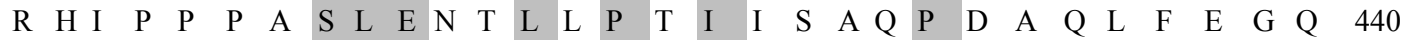

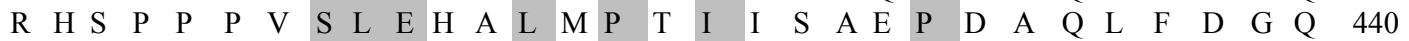

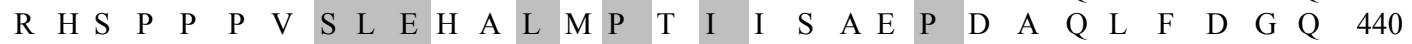
$\begin{array}{llllllllllllllllllllllllllllllll}\mathrm{R} & \mathrm{H} & \mathrm{S} & \mathrm{P} & \mathrm{P} & \mathrm{P} & \mathrm{V} & \mathrm{S} & \mathrm{L} & \mathrm{E} & \mathrm{H} & \mathrm{A} & \mathrm{L} & \mathrm{M} & \mathrm{P} & \mathrm{T} & \mathrm{I} & \mathrm{I} & \mathrm{S} & \mathrm{A} & \mathrm{E} & \mathrm{P} & \mathrm{D} & \mathrm{A} & \mathrm{Q} & \mathrm{L} & \mathrm{F} & \mathrm{D} & \mathrm{G} & \mathrm{Q} & 440\end{array}$ $\begin{array}{lllllllllllllllllllllllllllllll}\text { K } & \text { P } & \text { V } & \text { S } & \text { A } & \text { L } & \text { L } & \text { S } & \text { L } & \text { E } & \text { Q } & \text { A } & \text { L } & \text { L } & \text { P } & \text { A } & \text { I } & \text { L } & \text { P } & \text { S } & \text { R } & \text { P } & \text { S } & \text { D } & \text { A } & \text { A } & \text { D } & \text { E } & G & R & 446\end{array}$ $\begin{array}{llllllllllllllllllllllllllllllll}K & P & V & \text { S } & \text { A } & \text { P } & \text { L } & \text { S } & \text { L } & \text { E } & \text { Q } & \text { A } & \text { L } & \text { L } & \text { P } & \text { A } & \text { I } & \text { L } & \text { P } & \text { S } & \text { R } & \text { P } & \text { N } & \text { D } & \text { V } & \text { A } & \text { D } & \text { E } & \text { H } & \text { Q } & 446\end{array}$ $\begin{array}{llllllllllllllllllllllllllllllll}K & P & V & \text { S } & \text { A } & \text { P } & \text { L } & \text { S } & \text { L } & \text { E } & \text { Q } & \text { A } & \text { L } & \text { L } & \text { P } & \text { A } & \text { I } & \text { L } & \text { P } & \text { S } & \text { R } & \text { P } & \text { D } & \text { A } & \text { A } & \text { A } & \text { A } & \text { E } & \text { H } & \text { E } & 446\end{array}$ $\begin{array}{llllllllllllllllllllllllllllllll}\text { K } & \text { P } & \text { V } & \text { S } & \text { A } & \text { P } & \text { L } & \text { S } & \text { L } & \text { E } & \text { Q } & \text { A } & \text { L } & \text { L } & \text { P } & \text { A } & \text { I } & \text { L } & \text { P } & \text { S } & \text { R } & \text { P } & \text { S } & \text { D } & \text { A } & \text { A } & \text { D } & \text { E } & \text { G } & \text { T } & 446\end{array}$ $\begin{array}{llllllllllllllllllllllllllllllll}\text { K } & \text { P } & \text { V } & \text { P } & \text { A } & \text { P } & \text { L } & \text { S } & \text { L } & \text { E } & \text { Q } & \text { A } & \text { L } & \text { L } & \text { P } & \text { A } & \text { I } & \text { L } & \text { P } & \text { S } & \text { R } & \text { P } & \text { N } & \text { D } & \text { T } & \text { V } & \text { D } & \text { E } & \text { G } & \text { T } & 445\end{array}$ $\begin{array}{lllllllllllllllllllllllllllllll}\text { K } & \text { T } & \text { V } & \text { P } & \text { A } & \text { P } & \text { L } & \text { S } & \text { L } & \text { E } & \text { Q } & \text { A } & \text { L } & \text { L } & \text { P } & \text { A } & \text { I } & \text { L } & \text { P } & \text { S } & \text { R } & \text { P } & \text { N } & \text { D } & \text { T } & \text { V } & \text { D } & \text { E } & \text { G } & \text { T } & 445\end{array}$

$\begin{array}{lllllllllllllllllllllllllllllllll}\text { E } & E & E & E & Q & L & N & R & E & M & P & E & D & I & V & R & R & Q & L & I & N & N & L & A & K & H & V & L & F & T & 469\end{array}$

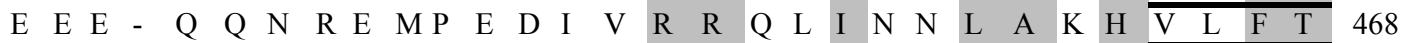

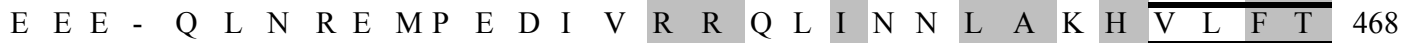
$\begin{array}{lllllllllllllllllllllllllllllll}\text { E } & E & E & - & Q & L & N & R & E & L & P & N & D & I & L & R & R & Q & L & I & N & N & L & A & K & H & \text { V } & \text { L } & \text { F } & \text { T } & 471\end{array}$ $\begin{array}{llllllllllllllllllllllllllllllllll}\text { Q } & E & Q & Q & Q & L & H & G & E & L & P & D & D & I & L & R & R & Q & I & I & N & S & L & A & K & H & V & I & F & T & 470\end{array}$ $\begin{array}{lllllllllllllllllllllllllllllll}\text { E } & \text { E E } & \text { - } & \text { Q } & \text { V } & \text { N } & \text { R } & \text { E } & \text { L } & \text { P } & \text { E } & \text { D } & \text { I } & \text { S } & \text { R } & \text { R } & \text { Q } & \text { L } & \text { I } & \text { N } & \text { N } & \text { L } & \text { A } & \text { K } & \text { H } & \text { V } & \text { L } & \text { F } & \text { T } & 469\end{array}$

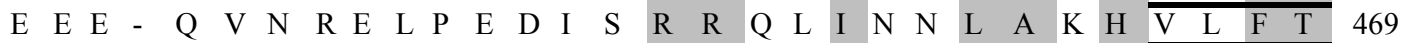

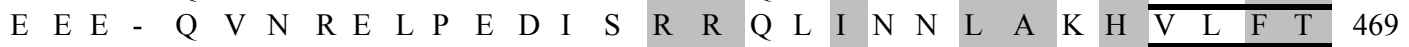
$\begin{array}{lllllllllllllllllllllllllllllllll}\mathrm{D} & \mathrm{T} & \mathrm{S} & \mathrm{I} & \mathrm{N} & \mathrm{E} & \mathrm{S} & \mathrm{R} & \mathrm{N} & \mathrm{A} & \mathrm{T} & \mathrm{D} & \mathrm{E} & \mathrm{S} & \mathrm{L} & \mathrm{R} & \mathrm{R} & \mathrm{R} & \mathrm{L} & \mathrm{I} & \mathrm{A} & \mathrm{N} & \mathrm{L} & \mathrm{A} & \mathrm{E} & \mathrm{H} & \mathrm{I} & \mathrm{L} & \mathrm{F} & \mathrm{T} & 476\end{array}$ $\begin{array}{lllllllllllllllllllllllllllllllll}\mathrm{D} & \mathrm{L} & \mathrm{S} & \mathrm{S} & \mathrm{N} & \mathrm{E} & \mathrm{S} & \mathrm{R} & \mathrm{N} & \mathrm{P} & \mathrm{A} & \mathrm{D} & \mathrm{E} & \mathrm{A} & \mathrm{F} & \mathrm{R} & \mathrm{R} & \mathrm{R} & \mathrm{L} & \mathrm{I} & \mathrm{A} & \mathrm{N} & \mathrm{L} & \mathrm{A} & \mathrm{E} & \mathrm{H} & \mathrm{I} & \mathrm{L} & \mathrm{F} & \mathrm{T} & 476\end{array}$

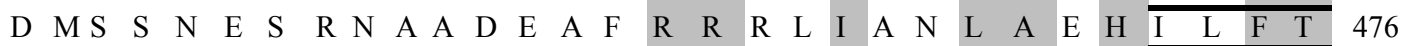
$\begin{array}{llllllllllllllllllllllllllllllll}\mathrm{D} & \mathrm{M} S & \mathrm{I} & \mathrm{N} & \mathrm{E} & \mathrm{S} & \mathrm{R} & \mathrm{N} & \mathrm{A} & \mathrm{T} & \mathrm{D} & \mathrm{E} & \mathrm{S} & - & \mathrm{R} & \mathrm{R} & \mathrm{R} & \mathrm{L} & \mathrm{I} & \mathrm{A} & \mathrm{H} & \mathrm{L} & \mathrm{A} & \mathrm{E} & \mathrm{H} & \mathrm{I} & \mathrm{L} & \mathrm{F} & \mathrm{T} & 476\end{array}$ $\begin{array}{lllllllllllllllllllllllllllllllll}\text { E } & A & S & L & P & N & S & R & D & I & T & S & E & P & Y & R & R & E & L & I & A & N & L & A & E & H & I & L & F & T & 475\end{array}$ $\begin{array}{llllllllllllllllllllllllllllllllll}\text { E } & A & S & L & P & N & S & K & D & I & T & S & E & P & Y & R & R & E & L & I & A & N & L & A & E & H & I & L & F & T & 475\end{array}$ 
C. aceratus

E. maclovinus

G. aculeatus

T. rubripes

M. zebra

P. nyererei

O. niloticus

H. sapiens

M. musculus

$R$. norvegicus

B. taurus

M. gallopavo

G. gallus

$N$. coriiceps

C. aceratus

E. maclovinus

G. aculeatus

T. rubripes

M. zebra

P. nyererei

O. niloticus

H. sapiens

M. musculus

$R$. norvegicus

B. taurus

M. gallopavo

G. gallus

N. coriiceps

C. aceratus

E. maclovinus

G. aculeatus

T. rubripes

M. zebra

P. nyererei

$O$. niloticus

H. sapiens

M. musculus

$R$. norvegicus

B. taurus

M. gallopavo

G. gallus

N. coriiceps

C. aceratus

E. maclovinus

G. aculeatus

T. rubripes

M. zebra

P. nyererei

O. niloticus

H. sapiens

M. musculus

$R$. norvegicus

B. taurus

M. gallopavo

G. gallus

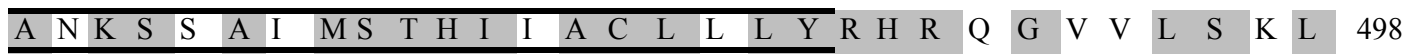
\begin{tabular}{llllllllllllllllllllllllllllllll}
\hline A & N & K & S & S & A & I & M S & T & H & I & I & A & C & L & L & L & Y & R & H & R & Q & G & V & G & L & S & K & L & 498
\end{tabular} \begin{tabular}{lllllllllllllllllllllllllllllll}
\hline A & N & $K$ & S & S & A & I & M S & T & H & I & V & A & C & L & L & L & Y & R & H & R & Q & G & V & V & L & S & K & L & 501
\end{tabular}

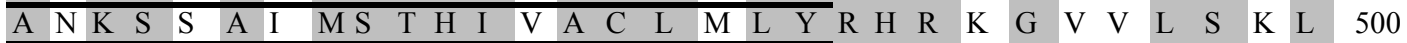
\begin{tabular}{lllllllllllllllllllllllllllllll}
\hline A & N K & S & S & A & I & M S & T & H & I & V & A & C & L & L & L & Y & R & H & R & Q & G & V & V & L & S & K & L & 499
\end{tabular}

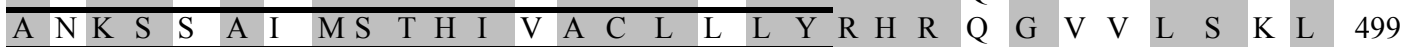

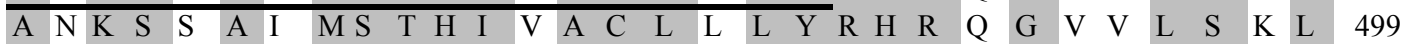
\begin{tabular}{lllllllllllllllllllllllllllllllll}
\hline A & S & K & S & C & A & I & M S & T & H & I & V & A & C & L & L & L & Y & $R$ & H & R & Q & G & I & D & L & S & T & L & 506
\end{tabular}

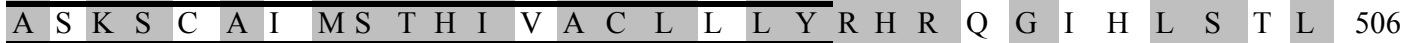

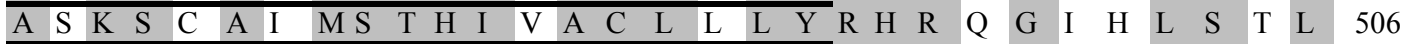

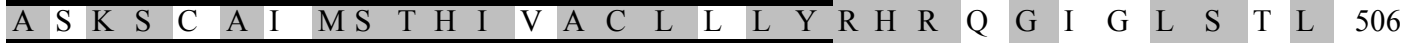

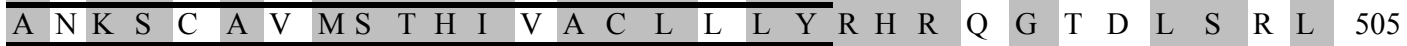

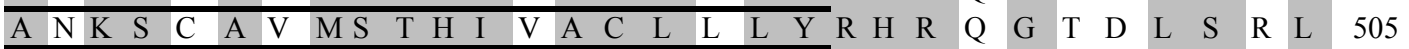

$\begin{array}{lllllllllllllllllllllllllllllllll}\text { V } & \text { E } & D & F & F & N & M & \text { K } & E & E & \text { I } & \text { L } & \text { S } & \text { R } & \text { D } & \text { F } & \text { D } & \text { L } & \text { G } & \text { F } & \text { S } & \text { G } & \text { N } & \text { S } & \text { E } & \text { D } & \text { V } & \text { V } & \text { V } & \text { R } & 529\end{array}$

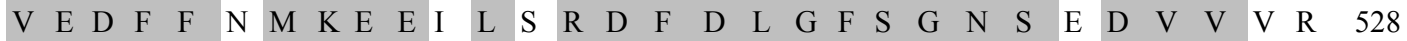

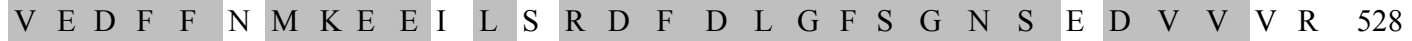
$\begin{array}{llllllllllllllllllllllllllllllll}\mathrm{V} & \mathrm{E} & \mathrm{D} & \mathrm{F} & \mathrm{F} & \mathrm{N} & \mathrm{M} & \mathrm{K} & \mathrm{E} & \mathrm{E} & \mathrm{I} & \mathrm{L} & \mathrm{S} & \mathrm{R} & \mathrm{D} & \mathrm{F} & \mathrm{D} & \mathrm{L} & \mathrm{G} & \mathrm{F} & \mathrm{S} & \mathrm{G} & \mathrm{N} & \mathrm{S} & \mathrm{E} & \mathrm{D} & \mathrm{V} & \mathrm{V} & \mathrm{M} & \mathrm{R} & 531\end{array}$ $\begin{array}{lllllllllllllllllllllllllllllll}V & E & D & F & F & S & M & K & E & E & I & L & S & R & D & F & D & L & G & F & S & G & N & S & E & D & V & V & M & R & 530\end{array}$ $\begin{array}{llllllllllllllllllllllllllllllll}\text { V } & E & D & F & F & N & M & K & E & E & I & L & S & R & D & F & D & L & G & F & S & G & N & S & E & D & V & V & M & R & 529\end{array}$ $\begin{array}{llllllllllllllllllllllllllllllll}V & E & D & F & F & N & M & K & E & E & I & L & S & R & D & F & D & L & G & F & S & G & N & S & E & D & V & V & M & R & 529\end{array}$ $\begin{array}{llllllllllllllllllllllllllllllll}\mathrm{V} & \mathrm{E} & \mathrm{D} & \mathrm{F} & \mathrm{F} & \mathrm{N} & \mathrm{M} & \mathrm{K} & \mathrm{E} & \mathrm{E} & \mathrm{I} & \mathrm{L} & \mathrm{S} & \mathrm{R} & \mathrm{D} & \mathrm{F} & \mathrm{D} & \mathrm{L} & \mathrm{G} & \mathrm{F} & \mathrm{S} & \mathrm{G} & \mathrm{N} & \mathrm{S} & \mathrm{E} & \mathrm{D} & \mathrm{V} & \mathrm{V} & \mathrm{M} & \mathrm{R} & 529\end{array}$ $\begin{array}{llllllllllllllllllllllllllllllll}V & E & D & F & F & V & M & K & E & E & V & L & A & R & D & F & D & L & G & F & S & G & N & S & E & D & V & V & M & H & 536\end{array}$ $\begin{array}{lllllllllllllllllllllllllllllll}V & E & D & F & F & V & M & K & E & E & V & L & A & R & D & F & D & L & G & F & S & G & N & S & E & D & V & V & M & H & 536\end{array}$

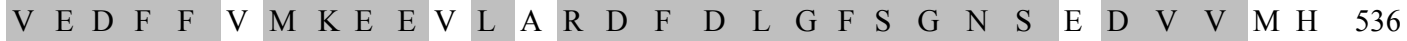

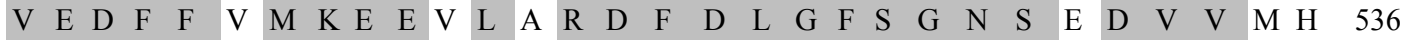

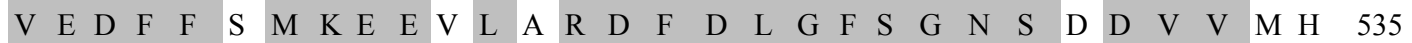

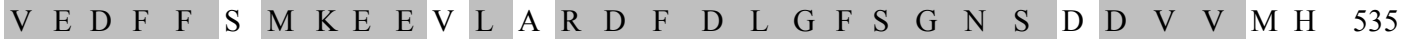

$\begin{array}{llllllllllllllllllllllllllllllll}\text { A } & \text { L } & \text { H } & \text { L } & \text { L } & G & N & \text { C } & \text { V } & \text { N V V } & \text { T } & \text { S } & \text { S } & \text { A } & \text { N } & \text { R } & \text { N } & G & \text { E } & \text { F } & \text { T } & \text { I } & \text { A } & \text { P } & \text { S } & \text { Q } & \text { T } & \text { V } & \text { P } & 559\end{array}$

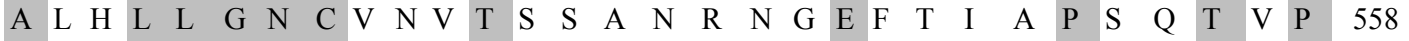
$\begin{array}{llllllllllllllllllllllllllllllll}\text { A } & \text { L } & H & \text { L } & \text { L } & G & N & C & V & N & V & T & \text { S } & \text { S } & \text { A } & \text { N } & R & \text { N } & G & \text { E } & \text { F } & \text { T } & \text { I } & \text { A } & \text { P } & \text { S } & \text { Q } & \text { T } & \text { V } & \text { P } & 558\end{array}$ $\begin{array}{llllllllllllllllllllllllllllllll}\text { A } & \text { L } & H & L & \text { L } & G & N & C & V & N & V & T & \text { S } & \text { S } & \text { S } & \text { N } & R & \text { N } & G & \text { E } & \text { F } & \text { T } & \text { I } & \text { A } & \text { P } & \text { S } & \text { Q } & \text { T } & \text { V } & \text { P } & 561\end{array}$ $\begin{array}{lllllllllllllllllllllllllllllll}\text { A } & \text { L } & H & L & L & G & N & C & V & N & V & T & \text { S } & \text { S } & \text { A } & \text { N } & \text { C } & \text { N } & G & \text { E } & \text { L } & \text { T } & \text { V } & \text { A } & \text { P } & \text { S } & \text { Q } & \text { T } & \text { V } & \text { P } & 560\end{array}$ $\begin{array}{lllllllllllllllllllllllllllllll}\text { A } & \text { L } & H & L & \text { L } & G & N & C & L & N & V & T & S & S & S & N & R & N & G & E & F & T & I & A & P & S & Q & T & V & P & 559\end{array}$ $\begin{array}{lllllllllllllllllllllllllllllll}\text { A } & \text { L } & \text { H } & \text { L } & \text { L } & G & \text { N } & \text { C } & \text { L } & \text { N V V } & \text { T } & \text { S } & \text { S } & \text { S } & \text { N } & \text { R } & \text { N } & G & \text { E } & \text { F } & \text { T } & \text { I } & \text { A } & \text { P } & \text { S } & \text { Q } & \text { T } & \text { V } & \text { P } & 559\end{array}$ $\begin{array}{lllllllllllllllllllllllllllllll}\text { A } & \text { L } & \text { H } & \text { L } & \text { L } & G & N & C & L & N & \text { V } & \text { T } & \text { S } & \text { S } & \text { S } & \text { N } & \text { R } & \text { N } & G & \text { E } & \text { F } & \text { T } & \text { I } & \text { A } & \text { P } & \text { T } & \text { Q } & \text { T } & \text { V } & \text { P } & 559\end{array}$ $\begin{array}{lllllllllllllllllllllllllllllll}\text { A } & \text { I } & \text { Q } & \text { L } & \text { L } & G & N & \text { C } & \text { V } & \text { T } & \text { I } & \text { T } & \text { H } & \text { T } & \text { S } & - & \text { R } & \text { N } & \text { D } & \text { E } & F & \text { F } & \text { I } & \text { T } & \text { P } & \text { S } & \text { T } & \text { T } & \text { V } & \text { P } & 565\end{array}$

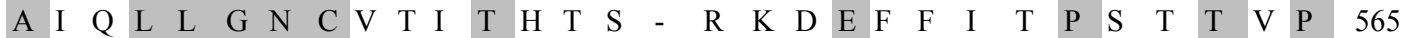
$\begin{array}{llllllllllllllllllllllllllllllll}\text { A } & \text { I } & \text { Q } & \text { L } & \text { L } & G & N & \text { C } & \text { V } & \text { T } & \text { I } & \text { T } & \text { H } & \text { T } & \text { S } & - & \text { R } & \text { K } & \text { D } & \text { E } & \text { F } & \text { F } & \text { I } & \text { T } & \text { P } & \text { S } & \text { T } & \text { T } & \text { V } & \text { P } & 565\end{array}$ $\begin{array}{llllllllllllllllllllllllllllllll}\text { A } & \text { I } & \text { Q } & \text { L } & \text { L } & G & \text { N } & \text { C } & \text { I } & \text { T } & \text { I } & \text { T } & \text { H } & \text { T } & \text { S } & - & \text { K } & \text { N } & \text { D } & \text { E } & \text { F } & \text { F } & \text { I } & \text { T } & \text { P } & \text { S } & \text { T } & \text { T } & \text { I } & \text { P } & 565\end{array}$ $\begin{array}{lllllllllllllllllllllllllllllll}\text { A } & \text { I } & \text { H } & \text { L } & \text { L } & G & N & C & V & N & I & T & N & T & S & - & R & N & N & E & F & F & \text { I } & \text { T } & \text { P } & \text { S } & \text { T } & \text { T } & \text { I } & \text { P } & 564\end{array}$

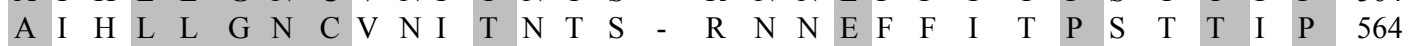

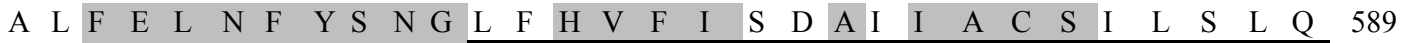
$\begin{array}{lllllllllllllllllllllllllllllllllll}\text { A } & \text { L } & \text { F } & \text { E } & \text { L } & \text { N } & \text { F } & \text { Y } & \text { S } & \text { N G } & \text { G } & \text { L } & \text { F } & \text { H } & \text { V } & \text { F } & \text { I } & \text { S } & \text { D } & \text { A I } & \text { I } & \text { A } & \text { C } & \text { S } & \text { I } & \text { L } & \text { S } & \text { L } & \text { Q } & 588\end{array}$ $\begin{array}{llllllllllllllllllllllllllllll}\text { A } & \text { L } & \text { F } & \text { E } & \text { L } & \text { N } & \text { F } & \text { Y } & \text { S } & \text { N G } & \text { G } & \text { L } & \text { F } & \text { H } & \text { V } & \text { F } & \text { I } & \text { S } & \text { D } & \text { A I } & \text { I } & \text { A } & \text { C } & \text { S } & \text { I } & \text { L } & \text { S } & \text { L } & \text { Q } & 588\end{array}$ $\begin{array}{llllllllllllllllllllllllllllll}\text { A } & \text { L } & \text { F } & \text { E } & \text { L } & \text { N } & \text { F } & \text { Y } & \text { S } & \text { N G } & \text { L } & \text { F } & \text { H } & \text { V } & \text { F } & \text { I } & \text { S } & \text { D } & \text { A I } & \text { I } & \text { A } & \text { C } & \text { S } & \text { I } & \text { L } & \text { S } & \text { L } & \text { Q } & 591 \\ 5\end{array}$ $\begin{array}{llllllllllllllllllllllllllllll}\text { A } & \text { L } & \text { F } & \text { E } & \text { L } & \text { N } & \text { F } & \text { Y } & \text { S } & \text { N G } & \text { G } & \text { L } & \text { F } & \text { H } & \text { V } & \text { F } & \text { I } & \text { S } & \text { D } & \text { A I } & \text { I } & \text { A } & \text { C } & \text { S } & \text { I } & \text { L } & \text { S } & \text { L } & \text { Q } & 590\end{array}$ $\begin{array}{llllllllllllllllllllllllllllll}\text { A } & \text { L } & \text { F } & \text { E } & \text { L } & \text { N } & \text { F } & \text { Y } & \text { S } & \text { N G } & \text { L } & \text { F } & \text { H } & \text { V } & \text { F } & \text { I } & \text { S } & \text { D } & \text { A } & \text { I } & \text { I } & \text { A } & \text { C } & \text { S } & \text { V } & \text { L } & \text { S } & \text { L } & \text { Q } & 589 \\ 5\end{array}$ \begin{tabular}{llllllllllllllllllllllllllllll} 
A & L & F & E & L & N & F & Y & S & N G & G & L & F & H & V & F & I & S & D & A I & I & A & C & S & V & L & S & L & Q & 589 \\
\hline
\end{tabular} \begin{tabular}{llllllllllllllllllllllllllllll} 
A & L & F & E & L & N & F & Y & S & N G & G & L & F & H & V & F & I & S & D & A & I & I & A & C & S & V & L & S & L & Q \\
\hline
\end{tabular}

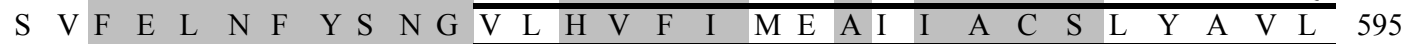

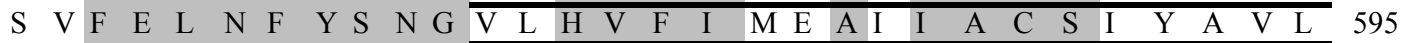

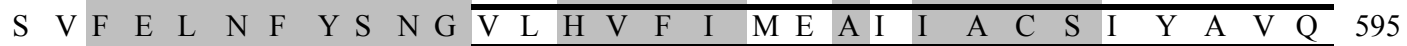

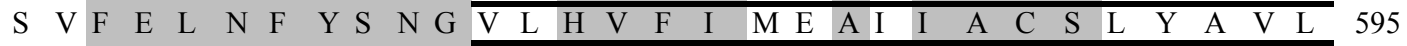
$\begin{array}{llllllllllllllllllllllllllllll}\text { A } & \text { V } & \text { F } & \text { E } & \text { L } & \text { N } & \text { F } & \text { Y } & \text { S } & \text { N G } & \text { I } & \text { L } & \text { H } & \text { V } & \text { F } & \text { I } & \text { K } & \text { E } & \text { A V V } & \text { I } & \text { A } & \text { C } & \text { S } & \text { L } & \text { R } & \text { A } & \text { V } & \text { Q } & 594\end{array}$

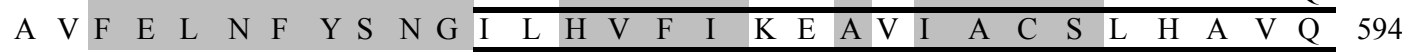


N. coriiceps

C. aceratus

E. maclovinus

G. aculeatus

T. rubripes

M. zebra

P. nyererei

$O$. niloticus

H. sapiens

M. musculus

$R$. norvegicus

B. taurus

M. gallopavo

G. gallus

N. coriiceps

C. aceratus

E. maclovinus

G. aculeatus

T. rubripes

M. zebra

P. nyererei

$O$. niloticus

H. sapiens

M. musculus

R. norvegicus

B. taurus

M. gallopavo

G. gallus

N. coriiceps

C. aceratus

E. maclovinus

G. aculeatus

T. rubripes

M. zebra

P. nyererei

O. niloticus

H. sapiens

M. musculus

R. norvegicus

B. taurus

M. gallopavo

G. gallus

N. coriiceps

C. aceratus

E. maclovinus

G. aculeatus

T. rubripes

M. zebra

P. nyererei

O. niloticus

H. sapiens

M. musculus

R. norvegicus

B. taurus

M. gallopavo

G. gallus

N. coriiceps $\begin{array}{llllllllllllllllllllllllllllllllllllll}\text { R } & \text { E } & \text { L } & \text { V } & \text { A } & \text { E } & \text { S } & \text { E } & \text { S } & \text { D } & H & \text { P } & \text { P } & \text { E } & C & \text { L } & \text { S } & \text { S } & \text { L } & \text { P } & \text { L } & \text { S } & \text { Q } & \text { E } & \text { R } & \text { L } & \text { I } & \text { R } & \text { K } & \text { A } & 619\end{array}$ $\begin{array}{lllllllllllllllllllllllllllllllllllllllll}R & E & L & V & A & E & S & E & S & D & P & P & P & E & G & L & S & S & L & P & L & S & Q & E & R & L & I & R & K & A & 618\end{array}$

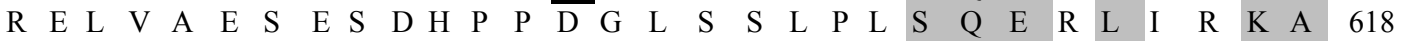
$\begin{array}{lllllllllllllllllllllllllllllllll}R & E & L & V & V & E & S & E & S & D & Q & P & A & D & G & P & S & S & L & P & L & S & Q & E & R & L & I & R & K & A & 621\end{array}$ $\begin{array}{lllllllllllllllllllllllllllllllll}R & E & L & I & A & E & S & E & S & D & H & L & P & A & D & I & N & S & L & P & L & S & Q & E & R & L & I & R & K & A & 620\end{array}$

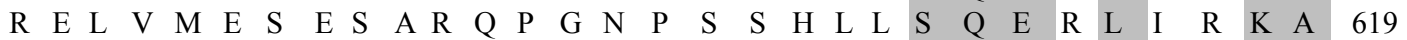
$\begin{array}{lllllllllllllllllllllllllllllllll}R & E & L & V & M & E & S & E & S & A & R & Q & P & G & N & P & S & S & H & L & L & S & Q & E & R & L & I & R & K & A & 619\end{array}$ $\begin{array}{lllllllllllllllllllllllllllllllll}R & E & L & V & I & E & S & E & S & A & R & Q & P & G & N & P & S & N & L & L & L & S & Q & E & R & L & I & R & K & A & 619\end{array}$ $\begin{array}{llllllllllllllllllllllllllllll} & \text { K R R G } & \text { L } & G & G & - & - & - & - & - & P & \text { T } & \text { S } & \text { T } & \text { P } & \text { P } & \text { N } & \text { L } & \text { I } & \text { S } & \text { Q } & \text { E } & \text { Q } & \text { L } & \text { V } & \text { R } & \text { K } & \text { A } & 620\end{array}$

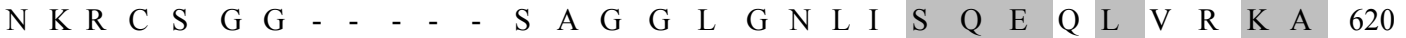

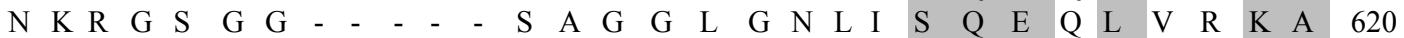
K K R G P G G - - - - - P A S - - P S L V S Q E Q L V H K A 618

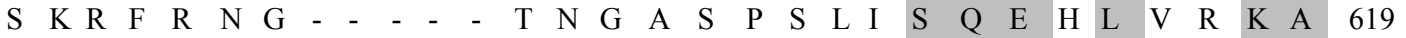

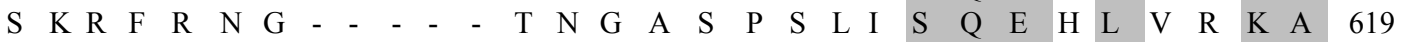

$\begin{array}{llllllllllllllllllllllllllllllllll}\text { A } & G & \text { L } & \text { S } & \text { H } & \text { F } & \text { L } & \text { V } & \text { N } & \text { E } & \text { V } & \text { T } & \text { L } & \text { A } & \text { P } & \text { P } & \text { C } & \text { Q } & \text { T } & \text { I } & \text { Y } & \text { Q } & \text { V } & \text { F } & \text { H } & \text { D } & \text { A } & \text { V } & \text { A } & R & 649\end{array}$ $\begin{array}{lllllllllllllllllllllllllllllllll}\text { A } & G & L & S & H & \text { F } & \text { L } & \text { V } & \text { N } & \text { E } & \text { V } & \text { T } & \text { L } & \text { A } & \text { P } & \text { P } & \text { C } & \text { Q } & \text { T } & \text { I } & \text { Y } & \text { Q } & \text { V } & \text { F } & \text { H } & \text { D } & \text { A } & \text { I } & \text { A } & R & 648\end{array}$ $\begin{array}{llllllllllllllllllllllllllllllllll}\text { A } & G & L & \text { S } & \text { H } & \text { F } & \text { L } & \text { V } & \text { N } & \text { E } & \text { V } & \text { T } & \text { L } & \text { A } & \text { P } & \text { P } & \text { C } & \text { Q } & \text { T } & \text { I } & \text { Y } & \text { Q } & \text { V } & \text { F } & \text { H } & \text { D } & \text { A } & \text { V } & \text { T } & R & 648\end{array}$ $\begin{array}{lllllllllllllllllllllllllllllllll}\text { A } & G & L & \text { S } & \text { H } & \text { F } & \text { L } & \text { V } & \text { N } & \text { E } & \text { V } & \text { S } & \text { V } & \text { A } & \text { P } & \text { P } & \text { C } & \text { Q } & \text { T } & \text { I } & \text { Y } & \text { Q } & \text { V } & \text { F } & \text { H } & \text { D } & \text { A } & \text { V } & \text { T } & R & 651\end{array}$ $\begin{array}{llllllllllllllllllllllllllllllll}\text { A } & G & L & S & Y & F & L & T & N & E & V & M & V & A & P & P & C & Q & T & L & Y & Q & V & F & H & D & A & V & T & R & 650\end{array}$ $\begin{array}{lllllllllllllllllllllllllllllllll}\text { A } & G & \text { L } & \text { S } & \text { H } & \text { F } & \text { L } & \text { I } & \text { N } & \text { E } & \text { V } & \text { A } & \text { V } & \text { A } & \text { P } & \text { P } & \text { C } & \text { Q } & \text { T } & \text { I } & \text { Y } & \text { Q } & \text { V } & \text { F } & \text { H } & \text { D } & \text { T } & \text { V } & \text { T } & \text { R } & 649\end{array}$ $\begin{array}{llllllllllllllllllllllllllllllll}\text { A } & G & L & \text { S } & \text { H } & \text { F } & \text { L } & \text { I } & \text { N } & \text { E } & \text { V } & \text { A } & \text { V } & \text { A } & \text { P } & \text { P } & \text { C } & \text { Q } & \text { T } & \text { I } & \text { Y } & \text { Q } & \text { V } & \text { F } & \text { H } & \text { D } & \text { T } & \text { V } & \text { T } & \text { R } & 649\end{array}$ $\begin{array}{llllllllllllllllllllllllllllllll}\text { A } & G & L & \text { S } & \text { H } & \text { F } & \text { L } & \text { I } & \text { N } & \text { E } & \text { V } & \text { A } & \text { V } & \text { A } & \text { P } & \text { P } & \text { C } & \text { Q } & \text { T } & \text { I } & \text { Y } & \text { Q } & \text { V } & \text { F } & \text { H } & \text { D } & \text { T } & \text { V } & \text { T } & R & 649\end{array}$

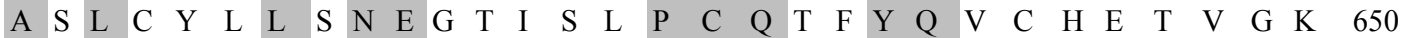
$\begin{array}{lllllllllllllllllllllllllllllll}\text { A } & \text { S } & \text { L } & \text { C } & \text { Y } & \text { L } & \text { L } & \text { S } & \text { N } & \text { E } & G & \text { T } & \text { I } & \text { S } & \text { L } & \text { P } & \text { C } & \text { Q } & \text { T } & \text { F } & \text { Y } & \text { Q } & \text { V } & \text { C } & \text { H } & \text { E } & \text { T } & \text { V } & \text { G } & \text { K } & 650\end{array}$ $\begin{array}{llllllllllllllllllllllllllllllll}\text { A } & \text { S } & \text { L } & \text { C } & \text { Y } & \text { L } & \text { L } & \text { S } & \text { N } & \text { E } & G & \text { T } & \text { I } & \text { S } & \text { L } & \text { P } & \text { C } & \text { Q } & \text { T } & \text { F } & \text { Y } & \text { Q } & \text { V } & \text { C } & \text { Q } & \text { E } & \text { T } & \text { V } & \text { G } & \text { K } & 650\end{array}$ $\begin{array}{llllllllllllllllllllllllllllllll}\text { A } & \text { S } & \text { L } & \text { C } & \text { Y } & \text { L } & \text { L } & \text { S } & \text { N } & \text { E } & G & \text { T } & \text { I } & \text { S } & \text { L } & \text { P } & \text { C } & \text { Q } & \text { T } & \text { F } & \text { Y } & \text { Q } & \text { I } & \text { C } & \text { H } & \text { E } & \text { T } & \text { V } & \text { G } & \text { R } & 648\end{array}$ $\begin{array}{llllllllllllllllllllllllllllllll}\text { A } & \text { S } & \text { L } & \text { C } & \text { Y } & \text { L } & \text { L } & \text { S } & \text { N } & \text { E } & \text { F } & \text { T } & \text { V } & \text { S } & \text { L } & \text { P } & \text { C } & \text { Q } & \text { L } & \text { I } & \text { Y } & \text { Q } & \text { V } & \text { C } & \text { H } & \text { E } & \text { A } & \text { V } & \text { E } & \text { K } & 649\end{array}$ $\begin{array}{llllllllllllllllllllllllllllllll}\text { A } & \text { S } & \text { L } & \text { C } & \text { Y } & \text { L } & \text { L } & \text { S } & \text { N } & \text { E } & \text { F } & \text { T } & \text { V } & \text { S } & \text { L } & \text { P } & \text { C } & \text { Q } & \text { L } & \text { I } & \text { Y } & \text { Q } & \text { V } & \text { C } & \text { H } & \text { E } & \text { A } & \text { V } & \text { E } & \text { K } & 649\end{array}$

$\begin{array}{lllllllllllllllllllllllllllllllll}\text { L } & \text { I } & \text { Q } & \text { Y } & G & \text { V } & \text { L } & \text { Y } & \text { V } & \text { A } & \text { E } & - & \text { E } & \text { D } & \text { Q } & \text { E } & \text { E } & \text { L } & \text { S } & \text { P } & \text { S } & \text { P } & \text { T } & \text { D } & \text { E } & \text { P } & \text { W } & \text { P } & \text { K } & \text { K } & 678\end{array}$ $\begin{array}{llllllllllllllllllllllllllllllll}\text { L } & \text { I } & Q & Y & G & \text { V } & \text { L } & \text { Y } & \text { V } & \text { A } & \text { E } & - & \text { E } & \text { D } & \text { Q } & \text { E } & \text { E } & \text { L } & \text { S } & \text { P } & \text { S } & \text { P } & \text { T } & \text { E } & \text { E } & \text { P } & \text { W } & \text { P } & \text { K } & \text { K } & 677\end{array}$ $\begin{array}{llllllllllllllllllllllllllllllll}\text { L } & \text { I } & Q & Y & G & V & L & Y & V & A & E & - & E & D & Q & E & E & L & S & P & S & P & T & E & E & P & \text { W } & P & K & K & 677\end{array}$

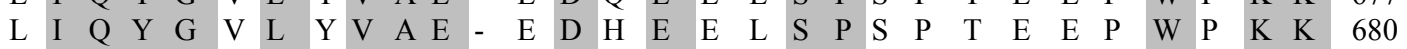
$\begin{array}{llllllllllllllllllllllllllllllll}\text { L } & \text { I } & \text { Q } & Y & G & \text { V } & \text { L } & \text { Y } & \text { V } & \text { A } & \text { E } & - & \text { E } & \text { D } & \text { Q } & \text { E } & \text { E } & \text { L } & \text { S } & \text { P } & \text { S } & \text { P } & \text { T } & \text { E } & \text { E } & \text { P } & \text { W } & \text { P } & \text { K } & \text { K } & 679\end{array}$

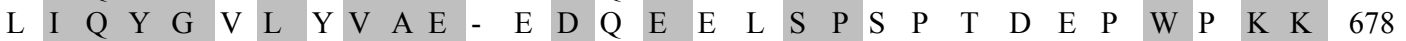

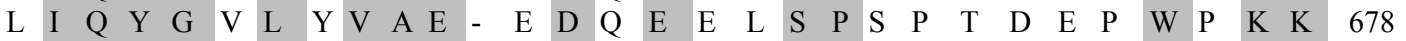

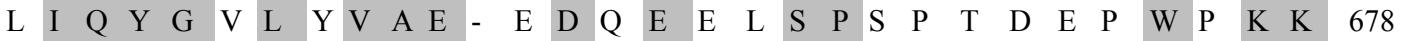

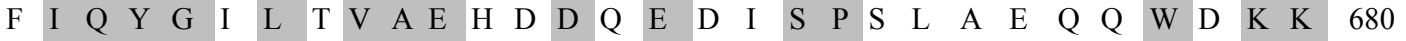
$\begin{array}{llllllllllllllllllllllllllllllll} & \text { I } & \text { I } & Q & Y & G & I & L & T & V & A & E & Q & D & D & Q & E & D & V & S & P & G & L & A & E & Q & Q & \text { W } & D & K & K & 680\end{array}$ $\begin{array}{llllllllllllllllllllllllllllllllll}\text { F } & \text { I } & Q & Y & G & I & L & T & V & A & E & Q & D & D & Q & E & D & V & S & P & G & L & A & E & Q & Q & \text { W } & \text { N } & \text { K } & \text { K } & 680\end{array}$

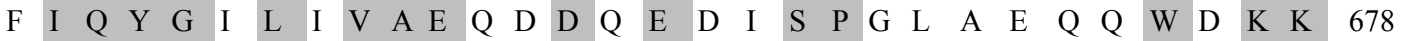
$\begin{array}{llllllllllllllllllllllllllllllllll}\text { L } & \text { I } & Q & Y & G & I & L & L & V & A & E & Q & D & D & Q & E & D & V & S & P & S & L & T & E & Q & Q & \text { W } & N & K & K & 679\end{array}$ $\begin{array}{lllllllllllllllllllllllllllllllll}\text { L } & \text { I } & Q & Y & G & I & L & L & V & A & E & Q & D & D & Q & E & D & V & S & P & S & L & T & E & Q & Q & \text { W } & \text { N } & K & K & 679\end{array}$

$\begin{array}{lllllllllllllllllllllllllllllllll}\text { F } & P & E & P & \text { L } & \text { S } & \text { W } & \text { R } & \text { S } & \text { D } & \text { E } & \text { E } & \text { D } & \text { E } & \text { D } & \text { S } & \text { D } & \text { F } & \text { G } & \text { E } & \text { E } & \text { Q } & \text { R } & \text { D } & \text { R } & \text { Y } & \text { L } & \text { K } & \text { V } & \text { S } & 708\end{array}$ $\begin{array}{lllllllllllllllllllllllllllllllll}F & P & E & P & L & S & \text { W } & R & S & D & E & E & D & E & D & S & D & F & G & E & E & Q & R & D & R & Y & L & K & V & S & 707\end{array}$

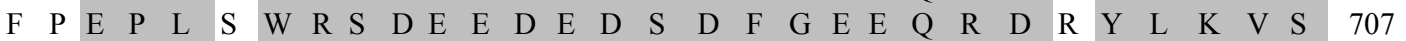

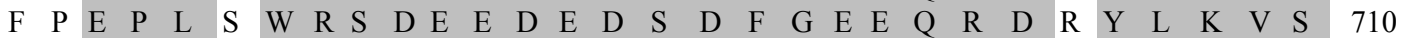
$\begin{array}{lllllllllllllllllllllllllllllllll}F & P & E & P & L & S & W & R & S & D & E & E & D & E & D & S & D & F & G & E & E & Q & R & D & R & Y & L & K & V & S & 709\end{array}$ $\begin{array}{llllllllllllllllllllllllllllllllll}\text { F } & A & E & P & L & S & \text { W } & R & S & D & E & E & D & E & D & S & D & F & G & E & E & Q & R & D & R & Y & L & K & V & S & 708\end{array}$ $\begin{array}{llllllllllllllllllllllllllllllll}\text { F } & A & E & P & L & S & \text { W } & R & S & D & E & E & D & E & D & S & D & F & G & E & E & Q & R & D & R & Y & L & K & V & S & 708\end{array}$

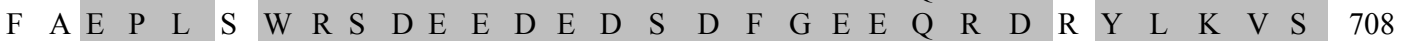

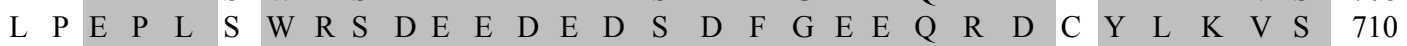

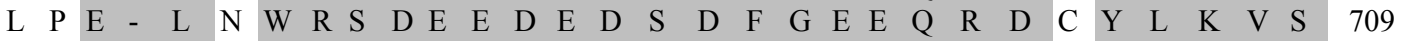

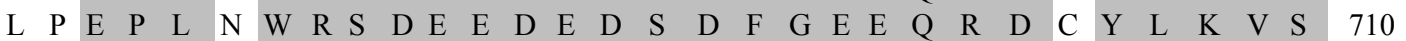

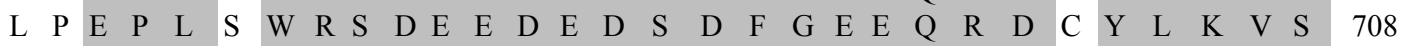
$\begin{array}{llllllllllllllllllllllllllllllll}\text { L } & \text { P } & \text { E } & \text { P } & \text { L } & \text { T } & \text { W } & \text { R } & \text { S } & \text { D } & \text { E } & \text { E } & \text { D } & \text { E } & \text { D } & \text { S } & \text { D } & \text { F } & \text { G } & \text { E } & \text { E } & \text { Q } & \text { R } & \text { D } & \text { C } & \text { Y } & \text { L } & \text { K } & \text { V } & \text { S } & 709\end{array}$

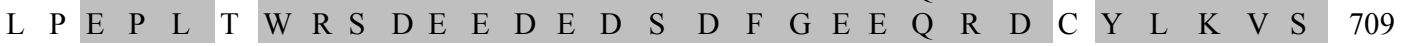


C. aceratus

E. maclovinus

G. aculeatus

T. rubripes

M. zebra

P. nyererei

O. niloticus

H. sapiens

M. musculus

$R$. norvegicus

B. taurus

M. gallopavo

G. gallus

N. coriiceps

C. aceratus

E. maclovinus

G. aculeatus

T. rubripes

M. zebra

$P$. nyererei

$O$. niloticus

H. sapiens

M. musculus

$R$. norvegicus

B. taurus

M. gallopavo

G. gallus

N. coriiceps

C. aceratus

E. maclovinus

G. aculeatus

T. rubripes

M. zebra

P. nyererei

O. niloticus

H. sapiens

M. musculus

$R$. norvegicus

B. taurus

M. gallopavo

G. gallus

$N$. coriiceps

C. aceratus

E. maclovinus

G. aculeatus

T. rubripes

M. zebra

P. nyererei

O. niloticus

H. sapiens

M. musculus

$R$. norvegicus

B. taurus

M. gallopavo

G. gallus $\begin{array}{lllllllllllllllllllllllllllllllll}\text { V } & \text { S } & \text { A } & \text { E } & \text { H } & \text { Q } & \text { E } & \text { F } & \text { F } & \text { V } & \text { F } & \text { L } & \text { Q } & \text { R } & \text { L } & \text { V } & \text { S } & \text { P } & \text { V } & \text { L } & \text { E } & \text { A } & \text { Y } & \text { S } & \text { G } & \text { A } & \text { A } & \text { I } & \text { F } & \text { V } & 737\end{array}$

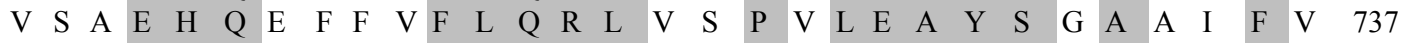

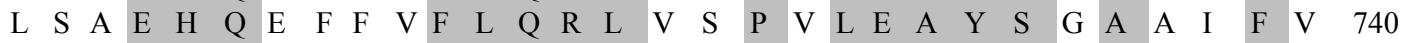
$\begin{array}{llllllllllllllllllllllllllllllll}\text { I } & \text { S } & A & E & H & \text { Q } & D & \text { F } & \text { F } & \text { I } & \text { F } & \text { L } & \text { Q } & \text { R } & \text { L } & \text { V } & \text { S } & \text { P } & \text { V } & \text { L } & \text { E } & \text { A } & \text { Y } & \text { S } & \text { G } & \text { A } & \text { A } & \text { I } & \text { F } & \text { I } & 739\end{array}$

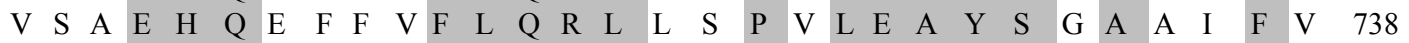
$\begin{array}{llllllllllllllllllllllllllllllll}\text { V } & \text { S } & A & \text { E } & \text { H } & \text { Q } & \text { E } & \text { F } & \text { F } & \text { V } & \text { F } & \text { L } & \text { Q } & \text { R } & \text { L } & \text { L } & \text { S } & \text { P } & \text { V } & \text { L } & \text { E } & \text { A } & \text { Y } & \text { S } & \text { G } & \text { A } & \text { A } & \text { I } & \text { F } & \text { V } & 738\end{array}$ $\begin{array}{lllllllllllllllllllllllllllllll}\text { V } & \text { S } & \text { A } & \text { E } & \text { H } & \text { Q } & \text { E } & \text { F } & \text { F } & \text { V } & \text { F } & \text { L } & \text { Q } & \text { R } & \text { L } & \text { L } & \text { S } & \text { P } & \text { V } & \text { L } & \text { E } & \text { A } & \text { Y } & \text { S } & \text { G } & \text { A } & \text { A } & \text { I } & \text { F } & \text { V } & 738\end{array}$ $\begin{array}{llllllllllllllllllllllllllllllll}\text { Q } & \text { S } & \text { K } & \text { E } & \text { H } & \text { Q } & \text { Q } & \text { F } & \text { I } & \text { T } & \text { F } & \text { L } & \text { Q } & \text { R } & \text { L } & \text { L } & \text { G } & \text { P } & \text { L } & \text { L } & \text { E } & \text { A } & \text { Y } & \text { S } & \text { S } & \text { A } & \text { A } & \text { I } & \text { F } & \text { V } & 740\end{array}$ $\begin{array}{lllllllllllllllllllllllllllllllll}\text { Q } & \text { S } & K & E & H & \text { Q } & \text { Q } & \text { F } & \text { I } & \text { T } & \text { F } & \text { L } & \text { Q } & \text { R } & \text { L } & \text { L } & \text { G } & \text { P } & \text { L } & \text { L } & \text { E } & \text { A } & \text { Y } & \text { S } & \text { S } & \text { A } & \text { A } & \text { I } & \text { F } & \text { V } & 739\end{array}$ $\begin{array}{lllllllllllllllllllllllllllllll}\text { Q } & \text { A } & K & E & H & \text { Q } & \text { Q } & \text { F } & \text { I } & \text { T } & \text { F } & \text { L } & \text { Q } & \text { R } & \text { L } & \text { L } & \text { G } & \text { P } & \text { L } & \text { L } & \text { E } & \text { A } & \text { Y } & \text { S } & \text { S } & \text { A } & \text { A } & \text { I } & \text { F } & \text { V } & 740\end{array}$ $\begin{array}{llllllllllllllllllllllllllllllll}\text { Q } & \text { S } & \text { K } & \text { E } & \text { H } & \text { Q } & \text { Q } & \text { F } & \text { I } & \text { T } & \text { F } & \text { L } & \text { Q } & \text { R } & \text { L } & \text { L } & \text { G } & \text { P } & \text { L } & \text { L } & \text { E } & \text { A } & \text { Y } & \text { S } & \text { S } & \text { A } & \text { A } & \text { V } & \text { F } & \text { I } & 738\end{array}$ $\begin{array}{lllllllllllllllllllllllllllllllll}\text { Q } & \text { S } & \text { Q } & \text { E } & \text { H } & \text { Q } & \text { Q } & \text { Y } & \text { I } & \text { T } & \text { F } & \text { L } & \text { Q } & \text { R } & \text { L } & \text { L } & \text { G } & \text { P } & \text { L } & \text { L } & \text { E } & \text { A } & \text { Y } & \text { S } & \text { S } & \text { A } & \text { V } & \text { I } & \text { F } & \text { V } & 739\end{array}$

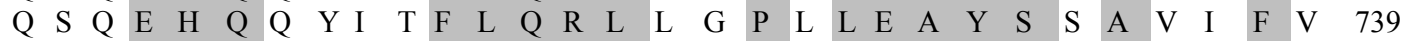

$\begin{array}{llllllllllllllllllllllllllllllllll}\text { H } & \text { S } & \text { L } & \text { S } & \text { Q } & \text { P } & \text { M } & \text { A } & \text { E } & \text { S } & \text { D } & \text { Y } & \text { T } & \text { Q } & \text { R } & \text { L } & \text { F } & \text { R } & \text { Y } & \text { L } & \text { L } & \text { T } & \text { R } & \text { T } & \text { E } & \text { R } & \text { G } & \text { V } & \text { A } & \text { A } & 768\end{array}$

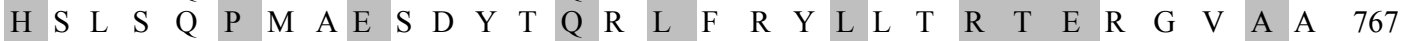
$\begin{array}{lllllllllllllllllllllllllllllllll}\text { H } & S & L & S & Q & P & M & A & E & S & D & Y & T & Q & R & L & F & R & Y & L & L & T & R & T & E & R & G & V & A & A & 767\end{array}$ $\begin{array}{llllllllllllllllllllllllllllllllll}\text { H } & S & L & S & Q & P & M & A & E & S & E & Y & T & Q & R & L & F & R & Y & L & L & T & R & T & E & R & G & V & A & A & 770\end{array}$ $\begin{array}{llllllllllllllllllllllllllllllll}\text { H } & \text { S } & \text { L } & \text { V } & \text { Q } & \text { P } & \text { T } & \text { S } & \text { E } & \text { S } & \text { D } & \text { Y } & \text { T } & \text { Q } & \text { K } & \text { L } & \text { F } & \text { R } & \text { Y } & \text { L } & \text { L } & \text { T } & \text { R } & \text { T } & \text { E } & \text { K } & \text { G } & \text { L } & \text { A } & \text { V } & 769\end{array}$ $\begin{array}{llllllllllllllllllllllllllllllll}\text { H } & \text { S } & \text { L } & \text { S } & \text { Q } & \text { P } & \text { M } & \text { V } & \text { E } & \text { S } & \text { D } & \text { Y } & \text { T } & \text { Q } & R & \text { L } & \text { F } & \text { R } & \text { Y } & \text { L } & \text { L } & \text { T } & \text { R } & \text { T } & \text { E } & \text { R } & \text { G } & \text { V } & \text { A } & \text { G } & 768\end{array}$

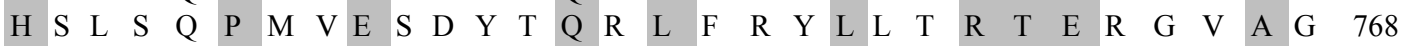
$\begin{array}{lllllllllllllllllllllllllllllllll}\text { H } & \text { S } & \text { L } & \text { S } & \text { Q } & \text { P } & \text { M } & \text { V } & \text { E } & \text { S } & \text { D } & \text { Y } & \text { T } & \text { Q } & \text { R } & \text { L } & \text { F } & \text { R } & \text { Y } & \text { L } & \text { L } & \text { T } & \text { R } & \text { T } & \text { E } & \text { R } & \text { G } & \text { V } & \text { A } & \text { G } & 768\end{array}$ $\begin{array}{lllllllllllllllllllllllllllllll}\text { H } & N & F & \text { S } & G & P & V & P & E & P & E & Y & L & Q & K & L & H & K & Y & L & I & T & R & T & E & R & N & V & A & V & 770\end{array}$ $\begin{array}{lllllllllllllllllllllllllllllllll}H & N & F & S & G & P & V & P & E & S & E & Y & L & Q & K & L & H & R & Y & L & I & T & R & T & E & R & N & V & A & V & 769\end{array}$ $\begin{array}{lllllllllllllllllllllllllllllll}\text { H } & \text { T } & F & R & G & P & V & P & E & S & E & Y & L & Q & K & L & H & R & Y & L & L & T & R & T & E & R & N & V & A & V & 770\end{array}$ $\begin{array}{llllllllllllllllllllllllllllllll}H & N & F & G & G & P & V & P & E & P & E & F & L & Q & K & L & H & K & Y & L & I & T & R & T & E & R & R & V & A & V & 768\end{array}$ $\begin{array}{lllllllllllllllllllllllllllllllll}\text { H } & N & F & S & G & P & V & \text { S } & E & S & E & Y & L & Q & K & L & H & R & H & L & I & S & R & T & E & K & N & V & A & V & 769\end{array}$ $\begin{array}{lllllllllllllllllllllllllllllllll}\text { H } & \text { N } & \text { F } & \text { S } & G & \text { P } & \text { V } & \text { S } & \text { E } & \text { S } & \text { E } & \text { Y } & \text { L } & \text { Q } & \text { K } & \text { L } & \text { H } & \text { R } & \text { H } & \text { L } & \text { I } & \text { N } & \text { R } & \text { T } & \text { E } & \text { K } & \text { N } & \text { V } & \text { A } & \text { V } & 769\end{array}$

$\begin{array}{llllllllllllllllllllllllllllllllll}\text { Y } & G & E & \text { S } & \text { A } & \text { T } & \text { H } & \text { Y } & \text { L } & \text { V } & \text { K } & \text { N } & \text { T } & \text { V } & \text { R } & \text { T } & \text { F } & \text { K } & \text { E } & \text { L } & \text { G } & \text { V } & \text { L } & \text { K } & \text { Q } & \text { R } & \text { K } & \text { E } & \text { N } & \text { Q } & 798\end{array}$ $\begin{array}{llllllllllllllllllllllllllllllll}\text { Y } & G & E & \text { S } & \text { A } & \text { T } & \text { H } & \text { Y } & \text { L } & \text { V } & \text { K } & \text { N } & \text { T } & \text { V } & \text { R } & \text { T } & \text { F } & \text { R } & \text { E } & \text { L } & \text { G } & \text { V } & \text { L } & \text { K } & \text { Q } & \text { R } & \text { K } & \text { E } & \text { N } & \text { Q } & 797\end{array}$ $\begin{array}{llllllllllllllllllllllllllllllll}\text { Y } & G & E & \text { S } & \text { A } & \text { T } & \text { H } & \text { Y } & \text { L } & \text { V } & \text { K } & \text { N } & \text { T } & \text { V } & \text { R } & \text { T } & \text { F } & \text { K } & \text { E } & \text { L } & \text { G } & \text { V } & \text { L } & \text { K } & \text { Q } & \text { R } & \text { R } & \text { E } & \text { N } & \text { Q } & 797\end{array}$ $\begin{array}{lllllllllllllllllllllllllllllll}Y & G & E & S & A & T & H & Y & L & V & K & N & T & V & K & T & F & K & E & L & G & V & L & K & Q & R & K & E & N & Q & 800\end{array}$ $\begin{array}{llllllllllllllllllllllllllllllll}\text { Y } & G & E & \text { S } & \text { A } & \text { T } & \text { H } & \text { Y } & \text { L } & \text { V } & \text { K } & \text { N } & \text { T } & \text { V } & \text { R } & \text { T } & \text { F } & \text { K } & \text { E } & \text { L } & \text { G } & \text { V } & \text { L } & \text { K } & \text { V } & \text { R } & \text { K } & \text { E } & \text { N } & \text { K } & 799\end{array}$ $\begin{array}{llllllllllllllllllllllllllllllll}\text { Y } & G & E & S & A & T & H & Y & L & V & K & N & T & V & R & T & F & K & E & L & G & V & L & K & E & R & R & E & N & K & 798\end{array}$ $\begin{array}{lllllllllllllllllllllllllllllllll}\text { Y } & G & E & \text { S } & \text { A } & \text { T } & \text { H } & \text { Y } & \text { L } & \text { V } & \text { K } & \text { N } & \text { T } & \text { V } & \text { R } & \text { T } & \text { F } & \text { K } & \text { E } & \text { L } & \text { G } & \text { V } & \text { L } & \text { K } & \text { E } & \text { R } & \text { R } & \text { E } & \text { N } & \text { K } & 798\end{array}$ $\begin{array}{llllllllllllllllllllllllllllllll}\text { Y } & G & E & S & A & T & H & Y & L & V & K & N & T & V & R & T & F & K & E & L & G & V & L & K & E & R & R & E & N & K & 798\end{array}$ $\begin{array}{llllllllllllllllllllllllllllllll}\text { Y } & \text { A } & \text { E } & \text { S } & \text { A } & \text { T } & \text { Y } & \text { C } & \text { L } & \text { V } & \text { K } & \text { N } & \text { A } & \text { V } & \text { K } & \text { M } & \text { F } & \text { K } & \text { D } & \text { I } & \text { G } & \text { V } & \text { F } & \text { K } & \text { E } & \text { T } & \text { K } & \text { Q } & \text { K } & R & 800\end{array}$

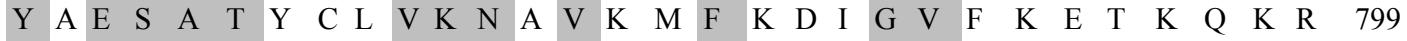
$\begin{array}{llllllllllllllllllllllllllllllll}\text { Y } & \text { A } & E & \text { S } & \text { A } & \text { T } & \text { Y } & \text { C } & \text { L } & \text { V } & \text { K } & \text { N } & \text { A } & \text { V } & \text { K } & \text { M } & \text { F } & \text { K } & \text { D } & \text { I } & \text { G } & \text { V } & \text { F } & \text { K } & \text { E } & \text { T } & \text { K } & \text { Q } & \text { K } & R & 800\end{array}$ $\begin{array}{llllllllllllllllllllllllllllllllll}\text { Y } & \text { A } & \text { E } & \text { S } & \text { A } & \text { T } & \text { Y } & \text { C } & \text { L } & \text { V } & \text { K } & \text { N } & \text { A } & \text { V } & \text { K } & \text { T } & \text { F } & \text { K } & \text { D } & \text { I } & \text { G } & \text { V } & \text { F } & \text { K } & \text { E } & \text { T } & \text { K } & \text { Q } & \text { K } & \text { R } & 798\end{array}$ $\begin{array}{llllllllllllllllllllllllllllllll}\text { Y } & \text { A } & \text { E } & \text { S } & \text { A } & \text { T } & \text { Y } & \text { S } & \text { H } & \text { V } & \text { K } & \text { N } & \text { A } & \text { V } & \text { K } & \text { V } & \text { F } & \text { K } & \text { E } & \text { I } & \text { G } & \text { V } & \text { F } & \text { S } & \text { Q } & \text { T } & \text { N } & \text { Q } & \text { K } & \text { K } & 799\end{array}$

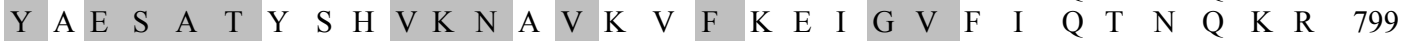

$\begin{array}{lllllllllllllllllllllllllllllllll}\text { V } & \text { T } & \text { T } & \text { L } & \text { E } & \text { L } & \text { S } & \text { S } & \text { T } & \text { F } & \text { L } & \text { P } & \text { Q } & \text { A } & \text { N } & \text { R } & \text { N } & \text { K } & \text { L } & \text { L } & \text { Q } & \text { Y } & \text { I } & \text { L } & \text { G } & \text { F } & \text { T } & \text { L } & \text { L } & & 827\end{array}$ $\begin{array}{lllllllllllllllllllllllllllllllll}\text { V } & \text { T } & \text { T } & \text { L } & \text { E } & \text { L } & \text { S } & \text { S } & \text { T } & \text { F } & \text { L } & \text { P } & \text { Q } & \text { A } & \text { N } & \text { R } & \text { N } & \text { K } & \text { L } & \text { L } & \text { Q } & \text { Y } & \text { I } & \text { L } & \text { G } & \text { F } & \text { A } & \text { L } & \text { L } & & 826\end{array}$ $\begin{array}{lllllllllllllllllllllllllllllllll}\text { V } & \text { T } & \text { T } & \text { L } & \text { E } & \text { L } & \text { S } & \text { S } & \text { T } & \text { F } & \text { L } & \text { P } & \text { Q } & \text { A } & \text { N } & \text { R } & \text { N } & \text { K } & \text { L } & \text { L } & \text { Q } & \text { Y } & \text { I } & \text { L } & \text { G } & \text { F } & \text { T } & \text { L } & \text { L } & & 826\end{array}$ $\begin{array}{llllllllllllllllllllllllllllllllll}\text { V } & \text { T } & \text { A } & \text { V } & \text { E } & \text { L } & \text { S } & \text { S } & \text { T } & \text { F } & \text { L } & \text { P } & \text { Q } & \text { A } & \text { N } & \text { R } & \text { N } & \text { K } & \text { L } & \text { L } & \text { H } & \text { Y } & \text { I } & \text { L } & \text { G } & \text { F } & \text { A } & \text { L } & \text { L } & & 829\end{array}$ $\begin{array}{lllllllllllllllllllllllllllllll}\text { M } & \text { T } & \text { I } & \text { L } & \text { E } & \text { L } & \text { S } & \text { S } & \text { T } & \text { F } & \text { L } & \text { P } & \text { Q } & \text { A } & \text { N } & \text { R } & \text { N } & \text { K } & \text { L } & \text { L } & \text { Q } & \text { Y } & \text { I } & \text { L } & \text { G } & \text { F } & \text { T } & \text { L } & \text { L } & & 828\end{array}$ $\begin{array}{llllllllllllllllllllllllllllllllll}\text { V } & \text { T } & \text { T } & \text { L } & \text { E } & \text { L } & \text { S } & \text { S } & \text { T } & \text { F } & \text { L } & \text { P } & \text { Q } & \text { E } & \text { N } & \text { R } & \text { N } & \text { K } & \text { L } & \text { L } & \text { Q } & \text { Y } & \text { I } & \text { L } & \text { G } & \text { F } & \text { T } & \text { L } & \text { L } & & 827\end{array}$ $\begin{array}{llllllllllllllllllllllllllllllllll}\mathrm{V} & \mathrm{T} & \mathrm{T} & \mathrm{L} & \mathrm{E} & \mathrm{L} & \mathrm{S} & \mathrm{S} & \mathrm{T} & \mathrm{F} & \mathrm{L} & \mathrm{P} & \mathrm{Q} & \mathrm{E} & \mathrm{N} & \mathrm{R} & \mathrm{N} & \mathrm{K} & \mathrm{L} & \mathrm{L} & \mathrm{Q} & \mathrm{Y} & \mathrm{I} & \mathrm{L} & \mathrm{G} & \mathrm{F} & \mathrm{T} & \mathrm{L} & \mathrm{L} & & 827\end{array}$ $\begin{array}{lllllllllllllllllllllllllllllllll}\mathrm{V} & \mathrm{T} & \mathrm{T} & \mathrm{L} & \mathrm{E} & \mathrm{L} & \mathrm{S} & \mathrm{S} & \mathrm{T} & \mathrm{F} & \mathrm{L} & \mathrm{P} & \mathrm{Q} & \mathrm{E} & \mathrm{N} & \mathrm{R} & \mathrm{N} & \mathrm{K} & \mathrm{L} & \mathrm{L} & \mathrm{Q} & \mathrm{Y} & \mathrm{I} & \mathrm{L} & \mathrm{G} & \mathrm{F} & \mathrm{T} & \mathrm{L} & \mathrm{L} & & 827\end{array}$ $\begin{array}{llllllllllllllllllllllllllllllllll}\text { V } & \text { S } & \text { V } & \text { L } & \text { E } & \text { L } & \text { S } & \text { S } & \text { T } & \text { F } & \text { L } & \text { P } & \text { Q } & \text { C } & \text { N } & \text { R } & \text { Q } & \text { K } & \text { L } & \text { L } & \text { E } & \text { Y } & \text { I } & \text { L } & \text { S } & \text { F } & \text { V } & \text { V } & \text { L } & & 829\end{array}$ $\begin{array}{lllllllllllllllllllllllllllllllll}\text { V } & \text { S } & \text { V } & \text { L } & \text { E } & \text { L } & \text { S } & \text { S } & \text { T } & \text { F } & \text { L } & \text { P } & \text { Q } & \text { C } & \text { N } & \text { R } & \text { Q } & \text { K } & \text { L } & \text { L } & \text { E } & \text { Y } & \text { I } & \text { L } & \text { S } & \text { F } & \text { V } & \text { V } & \text { L } & & 828\end{array}$ $\begin{array}{llllllllllllllllllllllllllllllll}\text { A } & \text { S } & \text { V } & \text { L } & \text { E } & \text { L } & \text { S } & \text { T } & \text { T } & \text { F } & \text { L } & \text { P } & \text { Q } & \text { G } & \text { S } & \text { R } & \text { Q } & \text { K } & \text { L } & \text { L } & \text { E } & \text { Y } & \text { I } & \text { L } & \text { S } & \text { F } & \text { V } & \text { V } & \text { L } & & 829\end{array}$ $\begin{array}{lllllllllllllllllllllllllllllllll}\text { V } & \text { S } & \text { V } & \text { L } & \text { E } & \text { L } & \text { S } & \text { N } & \text { T } & \text { F } & \text { L } & \text { P } & \text { Q } & \text { C } & \text { N } & \text { R } & \text { Q } & \text { K } & \text { L } & \text { L } & \text { E } & \text { Y } & \text { I } & \text { L } & \text { S } & \text { F } & \text { V } & \text { V } & \text { L } & & 827\end{array}$ $\begin{array}{lllllllllllllllllllllllllllllllll}\mathrm{D} & \mathrm{T} & \mathrm{I} & \mathrm{L} & \mathrm{E} & \mathrm{L} & \mathrm{S} & \mathrm{T} & \mathrm{T} & \mathrm{F} & \mathrm{L} & \mathrm{P} & \mathrm{Q} & \mathrm{R} & \mathrm{N} & \mathrm{R} & \mathrm{Q} & \mathrm{K} & \mathrm{L} & \mathrm{L} & \mathrm{E} & \mathrm{F} & \mathrm{I} & \mathrm{M} & \mathrm{S} & \mathrm{F} & \mathrm{M} & \mathrm{V} & \mathrm{L} & & 828\end{array}$ $\begin{array}{lllllllllllllllllllllllllllllllll}\mathrm{D} & \mathrm{T} & \mathrm{I} & \mathrm{L} & \mathrm{E} & \mathrm{L} & \mathrm{S} & \mathrm{T} & \mathrm{T} & \mathrm{F} & \mathrm{L} & \mathrm{P} & \mathrm{Q} & \mathrm{R} & \mathrm{N} & \mathrm{R} & \mathrm{Q} & \mathrm{K} & \mathrm{L} & \mathrm{L} & \mathrm{E} & \mathrm{F} & \mathrm{I} & \mathrm{M} & \mathrm{S} & \mathrm{F} & \mathrm{M} & \mathrm{V} & \mathrm{L} & & 828\end{array}$ 